\title{
Playing Games with Bounded Entropy
}

\author{
Mehrdad Valizadeh, Amin Gohari \\ Department of Electrical Engineering, Sharif University of Technology, Tehran, Iran \\ (valizadeh@ee.sharif.edu, aminzadeh@sharif.edu)
}

\begin{abstract}
In this paper, we consider zero-sum repeated games in which the maximizer (player or team) is restricted to strategies requiring no more than a limited amount of randomness. Particularly, we analyze the maxmin payoff of the maximizer in two models: the first model (the Neyman-Okada/Gossner-Vieille model) forces the maximizer to randomize her action in each stage just by conditioning her decision to the outcomes of a given sequence of random source, whereas in the second model (the Gossner and Tomala model), the maximizer is a team of players who are free to privately randomize their corresponding actions but do not have access to any explicit source of shared randomness needed for coordination. The works of Gossner and Vieille, and Gossner and Tomala adopted the method of types to establish their results; however, we utilize the idea of random hashing which is the core of randomness extractors in the information theory literature. In addition, we adopt the well-studied tool of simulation of a source from another source. By utilizing these tools, we are able to simplify the prior results and generalize them as well. We give a full characterization of the maxmin payoff of the maximizer in the repeated games under study. Particularly, the maxmin value of the first model is fully described by the function $\mathcal{J}(\mathrm{h})$, where $\mathcal{J}(\mathrm{h})$ is the maximum payoff that the maximizer can secure in the one-shot game by choosing mixed strategies of entropy at most $\mathrm{h}$. In the second part of the paper, we study the computational aspects of $\mathcal{J}(\mathrm{h})$, which has not received much attention in the game theory literature. We observe the equivalence of this problem with entropy minimization problems in other scientific contexts. Next, we offer three explicit lower bounds on the entropy-payoff trade-off curve. To do this, we provide and utilize
\end{abstract}


new results for the set of distributions that guarantee a certain payoff for Alice (mixed strategies corresponding to a security level for Alice). In particular, we study how this set of distributions shrinks as we increase the security level. While the use of total variation distance is common in game theory, our derivation indicates the suitability of utilizing the Rényi-divergence of order two.]

Keywords:

Repeated Games, Bounded Entropy, Randomness Extraction, Source Simulation, Entropy Minimization, Information Theory

\section{Contents}

1 Introduction

2 Preliminaries $\quad 9$

2.1 Notation . . . . . . . . . . . . . . . . . . . . . . . . . . . . 9

2.2 Entropy function . . . . . . . . . . . . . . . . . . 10

3 Repeated games with a bounded randomness source 11

3.1 Problem definition . . . . . . . . . . . . . . . . . 11

3.2 Simplifying the proof of Gossner and Vieille $(2002)$. . . . . . . . . . . . 13

3.3 Generalized model: leakage of the randomness source . . . . . . . . . . 17

3.3.1 Proof of Theorem 9; Alice can secure $\mathcal{J}_{\text {cav }}(H(X \mid Y)) \ldots \ldots$

3.3.2 Proof of Theorem 9; Bob can defend $\mathcal{J}_{\text {cav }}(H(X \mid Y)) \ldots \ldots$

4 Secret correlation in repeated games with imperfect monitoring 22

4.1 Problem definition . . . . . . . . . . . . . . . . . . . 23

4.2 Proof of Theorem 15: Payoff secured by team $A \ldots \ldots$. . . . . 25

4.3 Proof of Theorem 15: Player $B$ can defend $w \ldots \ldots$. . . . . . . . 33

A short version of this paper was presented at the 2017 IEEE Symposium On Information Theory (ISIT 2017). 


\section{Computation of min-entropy function}

5.1 Problem statement $\ldots \ldots \ldots \ldots \ldots$

5.2 On the set $\mathcal{P}_{\mathrm{U}}(w) \ldots \ldots \ldots \ldots \ldots \ldots \ldots \ldots$

5.2 .1 On the min-entropy function . . . . . . . . . . . . 40

5.3 Lower and upper bounds on the min-entropy function . . . . . . . . . . . 41

6 Proofs 47

6.1 Proof of Proposition $12 \ldots \ldots \ldots \ldots \ldots$

6.2 Proof of Proposition $17 \ldots \ldots \ldots \ldots \ldots$

6.3 Proof of Theorems 22 and $23 \ldots \ldots \ldots \ldots$

6.4 Proof of Theorem $25 \ldots \ldots \ldots \ldots \ldots$

6.5 Proof of Theorem $28 \ldots \ldots \ldots$

6.6 Proof of Theorem $32 \ldots \ldots \ldots \ldots \ldots$

6.7 Proof of Theorem $34 \ldots \ldots \ldots \ldots$

Appendix A Review of the randomness extraction literature 63

Appendix A.1 Conditional collision entropy ........ 63

Appendix A.2 Randomness extraction . . . . . . . . . . 65

Appendix B Proof of Proposition 20

Appendix C Proof of Lemma 39

\section{Introduction}

Nash (1950) proved that if the players of a given one-shot game can randomize on their pure strategies set according to any probability distribution, then the game has at least one Nash equilibrium in the mixed strategies. However, if the players have restrictions on implementing their random actions, then implementable Nash equilibria do not necessarily exist. Specifically, assume that one of the players is restricted to constructing her actions as a deterministic function of a given random source. In this setup, Nash equilibria do 
not necessarily exist. Similarly, in a class of repeated games including two-player zerosum games, Nash equilibria do not exist if sufficient random bits are not available to the players (See Hubáček et al. (2016) and Budinich and Fortnow (2011)). Therefore, when the players are limited to a given amount of randomness, the maximum payoff that each player can guarantee, regardless of what strategies the other players choose, becomes of interest. In this paper, we study the maximum guaranteed payoff in repeated zero-sum games with bounded randomness under two models. In the first model, we consider a finitely repeated version of a two-player zero-sum game in which the private randomness available to one of the players is limited. The second model is a zero-sum game between a team and an adversary player. In this game, the team players are free to randomize their corresponding actions privately but do not have access to any explicit source of shared randomness to coordinate their actions. It is assumed that the adversary player monitors the played actions imperfectly; hence, the history of actions observed by the team players is an implicit and limited random source for their coordination.

A version of the first model was studied by Gossner and Vieille (2002). They studied a repeated version of a zero-sum game $G$ between two players Alice and Bob, where Alice was the maximizer and Bob was the minimizer. At each stage, first, Alice observed an independent drawing of a random source $X$ whose distribution was a common knowledge. Both players then played an action which was monitored by the other player. Alice was restricted to choosing the action of each stage as a deterministic function of the observed random source up to that stage along with the history of the previous actions, while Bob chose his actions at each stage as a random function of the history of the previous actions. Note that the only source of randomization for Alice was the outcomes of random source $X$, while Bob could freely randomize his actions. Gossner and Vieille (2002) proved that when the number of stages of the repeated game is sufficiently large, the maximum expected average payoff of Alice is specified by the entropy-payoff trade-off curve of the one-shot game $G$. Specifically, for the one-shot game $G$, define $\mathcal{J}(\mathrm{h})$ as the maximum expected payoff that Alice can secure (regardless of what Bob plays) by playing mixed 
actions of entropy at most h. Let $\mathcal{J}_{\text {cav }}($.$) be the upper concave envelope of \mathcal{J}($.$) . Gossner$ and Vieille (2002) proved that the maximum expected average payoff of Alice in the repeated game converges to $\mathcal{J}_{\text {cav }}(H(X))$, where $H(X)$ is the entropy of random variable $X$. This is similar to Shannon's compression formula $H(X)$, which is defined on a single copy of the source (single letter), but gives the ultimate compression limit when multiple copies of the source are observed.

Generalizing the model of Gossner and Vieille (2002), we study a repeated zero-sum game that considers the possibility of leakage of Alice's random source sequence to Bob, and hence we call it the repeated game with leaked randomness source. In other words, we assume that Bob can imperfectly monitor the random source of Alice. More specifically, we assume an i.i.d. sequence of pairs $\left(X_{1}, Y_{1}\right),\left(X_{2}, Y_{2}\right), \ldots$ distributed according to a given distribution $p(x, y)$. The sequence of $X_{1}, X_{2}, \ldots$ is revealed symbol by symbol (causally) to Alice as the game is played out, while the sequence of $Y_{1}, Y_{2}, \ldots$ is revealed symbol by symbol to Bob. We can view $Y_{i}$ as the leakage that Bob obtains about Alice's observation. As before, Alice cannot randomize freely and is only able to use the randomness in the sequence of $X_{1}, X_{2}, \ldots$. We show that $\mathcal{J}_{\text {cav }}(H(X \mid Y))$ is the maximum payoff that Alice can secure regardless of Bob's actions. This result is a generalization of the result of Gossner and Vieille (2002), since when $Y$ is a constant random variable, the conditional entropy $H(X \mid Y)$ reduces to the unconditional entropy $H(X)$. Furthermore, it is obtained that if a genie provides the values of $Y_{1}, Y_{2}, \ldots$ symbol by symbol for Alice, the maximum payoff she can secure remains unchanged. In other words, knowledge of what Bob knows about Alice's observations is not helpful for Alice.

The second model, originally studied by Gossner and Tomala (2007), takes into account the limited access of a team players to shared randomness needed for coordination against an adversary. This model, which is called secret correlation in repeated games with imperfect monitoring, consists of a zero-sum game $G$ played repeatedly between team $A$ and player $B$. Team $A$, consisting of $m$ players, is the maximizer and player $B$ is the minimizer. At each stage, first, all players choose an action from their corresponding set 
of actions, and then the players of team $A$ observe all of the actions played, while player $B$ observes a noisy version of the action profile of team $A$. To choose the action of each stage, each player can privately randomize her action and utilize the history of her observations up to that stage. Since the action profile of team $A$ is revealed to player $B$ just through a noisy channel, the players of team $A$ could extract shared random bits from the profile of actions, where the extracted bits are almost independent of the observations of player $B$. These shared bits can be utilized by players of team $A$ to coordinate their actions in the upcoming stages. Although the players of team $A$ can randomize their actions privately and extract shared random bits, the extracted shared bits are limited. Consequently, in this setup, the set of implementable strategies of team $A$ is constrained by the amount of shared randomness they could extract and use.

In the model of Gossner and Tomala (2007), at each stage $t$, player $B$ monitors the action profile of team $A$ through a noisy channel with output signal $S_{t}$. They assumed that $S_{t}$ was not only seen by player $B$ but also by the players of team $A$. Generalizing the result of Gossner and Tomala (2007), we remove the assumption that the players of team $A$ observe $S_{t}$, and show that this causes no reduction in the payoff of team $A$.

The above generalizations of the results of Gossner and Vieille (2002) and Gossner and Tomala (2007) are immediate from our different proof technique. To explain this, consider that in order to construct the optimal strategies for the above two models of repeated games, we need to simulate random actions from the available source of randomness. In the first model, the source of randomness is the random sequence of $X_{1}, X_{2}, \ldots$; in the second model, the source of randomness is the history of (the imperfectly monitored) played actions. Gossner and Vieille (2002) and Gossner and Tomala (2007) introduced a new notion of absolute Kullback distance, which has not been used in the information theory literature. They took the absolute Kullback distance as the measure of the accuracy of the simulation, and utilized the method of types to simulate the desired random actions from the source of randomness. On the other hand, we consider the total variation distance as the measure of accuracy, and utilize the standard method of random hashing. 
The method of types alone is insufficient for obtaining the generalizations discussed in this paper, and hashing is necessary. We separate the simulation of random actions from the source of randomness into two steps: first, we extract sufficient private random bits from the randomness source, and then simulate the desired actions using the extracted random bits. Therefore, we need to use two tools for "randomness extraction" and "simulation of a source from another source". These tools are available in the literature of information theory for stationary or nonstationary/ergodic or non-ergodic sources (the information spectrum methods). Randomness extraction is reviewed in Appendix A of this paper for completeness. Through utilizing these tools, we are able to simplify the proofs and generalize the results of Gossner and Vieille (2002) and Gossner and Tomala (2007).

As stated above, the maximum guaranteed payoff of Alice in the repeated game with leaked randomness source (first model) is characterized in terms of $\mathcal{J}(\mathrm{h})$, which is the maximum payoff that Alice can secure in the one-shot game by choosing mixed actions with entropy at most h. In this paper, we also study the computational aspects of $\mathcal{J}(\mathrm{h})$. Equivalently, we study the inverse function of $\mathcal{J}(\mathrm{h})$ denoted by $F(w) . F(w)$ is the minimum entropy of the randomness consumed by Alice to guarantee payoff $w$ in the one-shot game. We call $F(w)$ the min-entropy function. 1

To compute $F(w)$, first, we need to consider the set of distributions on the action of Alice that would secure a payoff $w$ for her. This set will be a polytope in the space of all probability distributions. Then, we should solve an entropy minimization problem over this polytope in the space of probability distributions. In fact, minimizing and maximizing entropy arises in a wide range of contexts. Computing maximum entropy under a set of linear constraints is a well-studied problem with a wide range of applications, e.g., see Fang et al. (2012), (Cover and Thomas, 2012, p.367) and the principle of maximum entropy. Kapur et al. (1995) have shown that computing the minimum entropy can be also quite significant, and Watanabe (1981) has shown that many algorithms for clustering

\footnotetext{
${ }^{1}$ One should not confuse our "min-entropy function" with the term "min-entropy" commonly used to denote the Rényi entropy of order infinity.
} 
and pattern recognition are essentially solving entropy minimization problems. An special case of the entropy minimization problem (with its own applications) is that of finding a joint probability of minimum entropy given its marginal distributions (the marginal distribution is a linear constraint on the joint probability distribution); see Kocaoglu et al. (2017); Cicalese et al. (2017); Kovačević et al. (2015). In addition, Shor (2004) has shown that the quantum version of the entropy minimization problem is closely related to a number of noticeable problems in quantum information theory.

Kovačević et al. (2012) have shown that entropy minimization problem is an NPhard non-convex optimization problem. Since the entropy is a concave function over the probability simplex, its minimum occurs at a vertex of the feasible domain. As a result, computation of $F(w)$ leads to a search problem over an exponentially large set. Srikanth et al. (2003) have proposed an algorithm to solve the entropy minimization problem (and hence, can be used to compute $F(w)$ ), but it has no guarantee of finding the global minimum for all polytopes.

$F(w)$ provides a game theoretic interpretation of the entropy minimization problem. In Section 5, we study the properties of function $F(w)$, and utilize probabilistic tools to obtain a number of easy-to-compute bounds on the value of $F(w)$. While the literature on game theory makes extensive use of the total variation distance between distributions, we use the $\chi^{2}$-divergence (or the Tsallis divergence of order two) to derive a lower bound for $F(w)$. This lower bound is strictly tighter than the bound derived using the total variation distance showing the applicability of $\chi^{2}$-divergence in the context of game theory.

The rest of this paper is organized as follows: In Section 2, we introduce the notation of this paper, and present a concise discussion of Shannon entropy. In Section 3, we study a version of our first model studied by Gossner and Vieille (2002), and simplify the proof. The complete version of our first model namely the repeated game with leaked randomness source will be studied in Section 3.3. In Section 4, we investigate the problem of secret correlation in repeated games with imperfect monitoring, simplify the proof of Gossner and Tomala (2007), and extend their results. Section 5 is devoted to the computational 
aspects of the min-entropy function and in Section 6, we provide the proofs. Some of the details including a discussion on randomness extraction are left for the appendices.

\section{Preliminaries}

\subsection{Notation}

In this paper, we use the notation $x^{j}$ to represent a sequence of variables $\left(x_{1}, x_{2}, \ldots, x_{j}\right)$. The same notation is used to represent sequences of random variables, i.e., $X^{j}=\left(X_{1}, X_{2}, \ldots, X_{j}\right)$. Note that this notation is used for sequences that have two subscripts the same way i.e., $X_{k}^{j}=\left(X_{k, 1}, X_{k, 2}, \ldots, X_{k, j}\right)$. Calligraphic letters such as $\mathcal{X}, \mathcal{Y}, \mathcal{A}, \mathcal{B}, \ldots$ represent finite sets, and $|\mathcal{X}|$ denotes the cardinality of the finite set $\mathcal{X}$. Real vectors are represented by bold lower case letters, and bold uppercase letters are used to represent random vectors. For example the probability mass function (pmf) of a random variable $X$ with finite sample space $\mathcal{X}=\{1,2, \ldots, n\}$, beside the representation $p_{X}(x)$, will be also denoted by a vector $\mathbf{p}=\left(p_{1}, \ldots, p_{n}\right)$. When it is obvious from the context, we drop the subscript and use $p(x)$ instead of $p_{X}(x)$. We say that $X^{n}$ is drawn i.i.d. from $p(x)$ if

$$
p\left(x^{n}\right)=\prod_{i=1}^{n} p\left(x_{i}\right) .
$$

We use $\Delta(\mathcal{A})$ to denote the probability simplex on alphabet $\mathcal{A}$, i.e., the set of all probability distributions on the finite set $\mathcal{A}$. The total variation distance between pmfs $p_{X}$ and $q_{X}$ is denoted by $d_{1}\left(p_{X}, q_{X}\right)$ or $\left\|p_{X}-q_{X}\right\|_{T V}$ and is defined as:

$$
d_{1}\left(p_{X}, q_{X}\right)=\left\|p_{X}-q_{X}\right\|_{T V} \triangleq \frac{1}{2} \sum_{x \in \mathcal{X}}\left|p_{X}(x)-q_{X}(x)\right| .
$$

When the pmfs are represented by vectors $\mathbf{p}$ and $\mathbf{q}$, the total variation distance between them is represented by $d_{1}(\mathbf{p}, \mathbf{q})$ or $\|\mathbf{p}-\mathbf{q}\|_{T V}$. Some of the properties of the total variation distance are summarized in the following lemma.

Lemma 1. The following properties hold for the total variation distance:

Property 1: $\left\|p_{E} p_{F \mid E}-q_{E} p_{F \mid E}\right\|_{T V}=\left\|p_{E}-q_{E}\right\|_{T V}$ 
Property 2: $\left\|p_{E} p_{F \mid E}-q_{E} q_{F \mid E}\right\|_{T V} \geq\left\|p_{E}-q_{E}\right\|_{T V}$

Property 3: $\left\|p_{E_{1}} p_{F_{1}}-p_{E_{2}} q_{F_{2}}\right\|_{T V} \leq\left\|p_{E_{1}}-p_{E_{2}}\right\|_{T V}+\left\|p_{F_{1}}-q_{F_{2}}\right\|_{T V}$;

Property 4: For an arbitrary deterministic function $f$ on the sample space of $E_{1}$ and $E_{2}$ we have $\left\|p_{f\left(E_{1}\right)}-p_{f\left(E_{2}\right)}\right\|_{T V} \leq\left\|p_{E_{1}}-p_{E_{2}}\right\|_{T V}$;

Property 5: $\left\|p_{E_{1}} p_{F}-p_{E_{2}} p_{F}\right\|_{T V}=\left\|p_{E_{1}}-p_{E_{2}}\right\|_{T V}$.

\subsection{Entropy function}

Let $X \in \mathcal{X}$ and $Y \in \mathcal{Y}$ be two random variables with joint probability distribution $p_{X, Y}$ and respective marginal distributions $p_{X}$ and $p_{Y}$. The Shannon entropy (or simply the entropy) of the random variable $X$ is defined to be:

$$
H(X)=\sum_{x \in \mathcal{X}}-p_{X}(x) \log \left(p_{X}(x)\right),
$$

where $0 \log (0)=0$ by continuity and all logarithms in this paper are in base two. Since the entropy is a function of the $\operatorname{pmf} p_{X}$, we sometimes write $H\left(p_{X}\right)$ (or $H(\mathbf{p})$ when the pmf is denoted by probability vector $\mathbf{p}$ ) instead of $H(X)$.

The conditional Shannon entropy (or simply the conditional entropy) of $X$ given $Y$ is defined as:

$$
\begin{aligned}
H(X \mid Y) & =\sum_{(x, y) \in \mathcal{X} \times \mathcal{Y}}-p_{X Y}(x, y) \log \left(p_{X \mid Y}(x \mid y)\right) \\
& =\sum_{y \in \mathcal{Y}} p_{Y}(y) H(X \mid Y=y),
\end{aligned}
$$

where $H(X \mid Y=y)=\sum_{x \in \mathcal{X}}-p_{X \mid Y}(x \mid y) \log \left(p_{X \mid Y}(x \mid y)\right)$.

Using the definition of entropy and conditional entropy one can check that

$$
H(X, Y)=H(Y)+H(X \mid Y) .
$$

Utilizing the above property iteratively, the chain rule for a sequence of random variables is obtained:

$$
H\left(X^{n}\right)=H\left(X_{1}\right)+\sum_{i=2}^{n} H\left(X_{i} \mid X^{i-1}\right) .
$$

Furthermore, the following properties hold for the entropy function: 
- $H(X) \geq 0$.

- $H\left(X_{1}, X_{2}\right) \geq H\left(X_{1}\right)$.

- Let $f(x)$ be an arbitrary deterministic function then $H(f(X)) \leq H(X)$.

\section{Repeated games with a bounded randomness source}

In this section, we revisit the repeated game studied by Gossner and Vieille (2002). The high-level picture of the proof of Gossner and Vieille (2002) is as follows: it uses the so-called "block-Markov" proof technique of information theory, where we divide time into a number of blocks and Alice uses her observations in each block to produce the actions for the next block. Our goal is to show that the high-level picture of the proof presented in Gossner and Vieille (2002) can be made precise in an easier manner using standard information theory tools.

\subsection{Problem definition}

Let us first begin with reviewing the definition of the repeated game with a bounded randomness source studied by Gossner and Vieille (2002). Consider a $T$ stage repeated zero-sum game between players $\operatorname{Alice}(A)$ and $\operatorname{Bob}(B)$ with respective finite action sets $\mathcal{A}=\{1, \ldots, n\}$ and $\mathcal{B}=\left\{1, \ldots, n^{\prime}\right\}$, where $n$ and $n^{\prime}$ are natural numbers. Let $X^{T}=$ $\left(X_{1}, X_{2}, \ldots, X_{T}\right)$ be a sequence of random variables drawn i.i.d. from a sample space $\mathcal{X}$ with law $p_{X}$. In every stage $t \in\{1,2, \ldots, T\}$, first, Alice observes random source $X_{t}$ privately, and then, Alice and Bob choose actions $A_{t} \in \mathcal{A}$ and $B_{t} \in \mathcal{B}$, respectively. At the end of stage $t$, both players observe the chosen actions $A_{t}$ and $B_{t}$ and Alice gets stage payoff $u_{A_{t}, B_{t}}$ from Bob. In order to choose actions at stage $t$, players make use of the history of their observations up to stage $t$, which is denoted by $\mathrm{H}_{1}^{t}=\left(X_{1}, A_{1}, B_{1}, \ldots, X_{t-1}, A_{t-1}, B_{t-1}, X_{t}\right)$ for Alice, and $\mathrm{H}_{2}^{t}=\left(A_{1}, B_{1}, \ldots, A_{t-1}, B_{t-1}\right)$ for Bob. Let $\sigma_{t}:(\mathcal{A} \times \mathcal{B})^{t-1} \times \mathcal{X}^{t} \rightarrow \mathcal{A}$ and $\tau_{t}:(\mathcal{A} \times \mathcal{B})^{t-1} \rightarrow \mathcal{B}$ be the functions mapping the history of observations of Alice and Bob to actions at stage $t$, so $A_{t}=\sigma_{t}\left(\mathrm{H}_{1}^{t}\right)$ and $B_{t}=\tau_{t}\left(\mathrm{H}_{2}^{t}\right)$. Note that Alice does not have access to any private source of randomness 
except $\mathbf{H}_{1}^{t}$, so she has to use the deterministic function $\sigma_{t}(\cdot)$, while Bob can utilize the random function $\tau_{t}(\cdot)$. We call the $T$-tuples $\sigma=\left(\sigma_{1}, \sigma_{2}, \ldots, \sigma_{T}\right)$ and $\tau=\left(\tau_{1}, \tau_{2}, \ldots, \tau_{T}\right)$ the strategies of Alice and Bob, respectively. The expected average payoff for Alice up to stage $T$ induced by strategies $\sigma$ and $\tau$ is denoted by $\lambda_{T}(\sigma, \tau)$, which is

$$
\lambda_{T}(\sigma, \tau)=\mathbb{E}_{\sigma, \tau}\left[\frac{1}{T} \sum_{t=1}^{T} u_{A_{t}, B_{t}}\right],
$$

where $\mathbb{E}_{\sigma, \tau}$ denotes the expectation with respect to the distribution induced by i.i.d. repetitions of $p_{X}$ and strategies $\sigma$ and $\tau$. Alice wishes to maximize $\lambda_{T}(\sigma, \tau)$, and Bob wishes to minimize it.

Definition 2. Let $v$ be an arbitrary real value:

- Alice can secure $v$ if there exists a strategy $\sigma^{*}$ for Alice such that for all strategy $\tau$ of Bob we have $\liminf _{T \rightarrow \infty} \lambda_{T}\left(\sigma^{*}, \tau\right) \geq v$.

- Bob defends $v$ if given an arbitrary strategy $\sigma$ for Alice, there exists a strategy $\tau^{*}$ for Bob such that $\lim \sup _{T \rightarrow \infty} \lambda_{T}\left(\sigma, \tau^{*}\right) \leq v$.

- $v$ is the maxmin value of the repeated game, if Alice can secure $v$ and Bob can defend $v$.

Theorem 3 (Gossner and Vieille (2002)). The maxmin value of the repeated game with bounded randomness source defined in Section 3.1 is $\mathcal{J}_{\text {cav }}(H(X))$, where $\mathcal{J}_{\text {cav }}(\mathrm{h})$ is the upper concave envelope of

$$
\mathcal{J}(\mathrm{h})=\max _{\mathbf{p} \in \Delta(\mathcal{A}), H(\mathbf{p}) \leq \mathbf{h}} \min _{b \in \mathcal{B}} \mathbb{E}_{\mathbf{p}}\left[u_{A, b}\right]
$$

where $\mathbb{E}_{\mathbf{p}}$ denotes the expectation with respect to $\mathbf{p}$.

In Section 3.2, we give a simplified proof of Gossner and Vieille (2002) that explains how Alice can secure $\mathcal{J}_{\text {cav }}(H(X))$. The complete proof is left for Section 3.3, where we present a generalized version of the above result. 


\subsection{Simplifying the proof of Gossner and Vieille (2002)}

As mentioned in Gossner and Vieille (2002), the upper concave envelope $\mathcal{J}_{\text {cav }}(\cdot)$ at $\mathrm{h}=H(X)$ can be expressed as the convex combination

$$
\gamma \mathcal{J}\left(H\left(p_{A}^{(1)}\right)\right)+(1-\gamma) \mathcal{J}\left(H\left(p_{A}^{(2)}\right)\right)
$$

for some $\gamma \in[0,1]$ and $\operatorname{pmfs} p_{A}^{(1)}$ and $p_{A}^{(2)}$ in $\Delta(\mathcal{A})$, where $p_{A}^{(1)}$ and $p_{A}^{(2)}$ secure respective payoffs $\mathcal{J}\left(H\left(p_{A}^{(1)}\right)\right)$ and $\mathcal{J}\left(H\left(p_{A}^{(2)}\right)\right)$ in the one-shot game and the following equality is satisfied:

$$
\gamma H\left(p_{A}^{(1)}\right)+(1-\gamma) H\left(p_{A}^{(2)}\right)=H(X)
$$

As a result, it suffices to show that for any $p_{A}^{(1)}$ and $p_{A}^{(2)}$ satisfying

$$
\gamma H\left(p_{A}^{(1)}\right)+(1-\gamma) H\left(p_{A}^{(2)}\right)<H(X)
$$

Alice can use the source of randomness $X$ to secure payoffs arbitrarily close to

$$
\gamma \min _{b \in \mathcal{B}} \mathbb{E}_{p_{A}^{(1)}}\left[u_{A, b}\right]+(1-\gamma) \min _{b \in \mathcal{B}} \mathbb{E}_{p_{A}^{(2)}}\left[u_{A, b}\right]
$$

which is the weighted average of the payoffs that input distributions $p_{A}^{(1)}$ and $p_{A}^{(2)}$ secure.

Let the game be played for $T$ stages. Take some $T$ of the form $T=N L$, and divide the total $T$ stages into $N$ blocks of length $L$. Excluding the first block, we generate the action sequence of each block as a function of the random source observed during the previous block (we ignore the payoff of the first block throughout the discussion, since by taking the number of blocks $N$ large enough, the contribution of the first block in Alice's net average payoff becomes negligible). More specifically, excluding the first block, each block is further divided into two subblocks, where the first subblock takes up $\gamma$ fraction of the block as illustrated in Fig. 1. Alice aims to use her observed random source during the previous block to play almost i.i.d. according to $p_{A}^{(1)}$ during the first subblock and according to $p_{A}^{(2)}$ during the second subblock. In addition, Alice wants her action at any given stage to be also almost independent of Bob's observations up to that stage. Observe that if Alice could produce actions that were perfectly i.i.d. according to $p_{A}^{(1)}$ in the first 

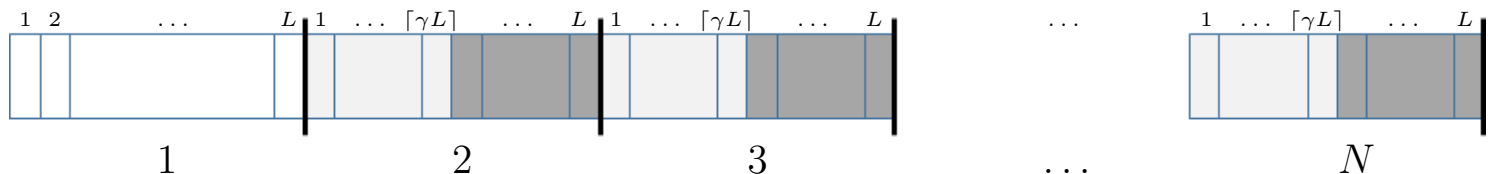

$N$

Time slots of the first block in which Alice plays the pure action $1 \in \mathcal{A}$

Time slots in which Alice draws a random action with law $p_{A}^{(1)}$

Time slots in which Alice draws a random action with law $p_{A}^{(2)}$

Figure 1: Illustration of the block Markov strategy

subblock and perfectly i.i.d. according to $p_{A}^{(2)}$ in the second subblock, both independent of Bob's observations, then in $\gamma$ fraction of the stages in a block, she would secure the average payoff of $\min _{b \in \mathcal{B}} \mathbb{E}_{p_{A}^{(1)}}\left[u_{A, b}\right]$ per action, and in the remaining $1-\gamma$ fraction she would secure the payoff of $\min _{b \in \mathcal{B}} \mathbb{E}_{p_{A}^{(2)}}\left[u_{A, b}\right]$. This would give Alice a total payoff of

$$
\gamma \min _{b \in \mathcal{B}} \mathbb{E}_{p_{A}^{(1)}}\left[u_{A, b}\right]+(1-\gamma) \min _{b \in \mathcal{B}} \mathbb{E}_{p_{A}^{(2)}}\left[u_{A, b}\right]
$$

We will now show that regarding the total variation distance as the measure of accuracy, Alice can play almost i.i.d. according to $p_{A}^{(1)}$ during the first subblock of each block, and almost i.i.d. according to $p_{A}^{(2)}$ during the second subblock of each block. Therefore, Alice can secure payoffs arbitrarily close to $\gamma \min _{b \in \mathcal{B}} \mathbb{E}_{p_{A}^{(1)}}\left[u_{A, b}\right]+(1-\gamma) \min _{b \in \mathcal{B}} \mathbb{E}_{p_{A}^{(2)}}\left[u_{A, b}\right]$ and this will complete the proof.

Remember that $\gamma H\left(p_{A}^{(1)}\right)+(1-\gamma) H\left(p_{A}^{(2)}\right)<H(X)$. Intuitively speaking, since Alice's observation from a block has entropy $L H(X)$, which is larger than the entropy of her intended action in the next block $L \gamma H\left(p_{A}^{(1)}\right)+L(1-\gamma) H\left(p_{A}^{(2)}\right)$, she should be able to find a proper mapping to produce her actions in the current block from her observations in the preceding block. Gossner and Vieille (2002) propose such a mapping and put effort to prove its correctness. This is where most of the effort is spent. The essential problem here is to simulate a source from another source (here the observations from one block to actions in the next block). This problem is solved in the information theory literature. As stated below in Lemma 6, which is adopted from ( $\mathrm{Han}, 2003$, p. 110), the measure of relevance to compare between the two sources is inf-entropy and sup-entropy, rather 
than entropy. However, for i.i.d. sources (or concatenation of i.i.d. sources), inf-entropy and sup-entropy reduce to the normal Shannon entropy, as discussed in Remark 5 below.

Definition 4. Sup-entropy and inf-entropy of a random source $Z_{1}, Z_{2}, \ldots$ are denoted by $\underline{H}(Z)$ and $\bar{H}(Z)$, respectively, and defined as follows:

$$
\begin{aligned}
& \underline{H}(Z)=p-\liminf _{L \rightarrow \infty} \frac{1}{L} \log \frac{1}{p_{Z^{L}}\left(Z^{L}\right)}, \\
& \bar{H}(Z)=p-\limsup _{L \rightarrow \infty} \frac{1}{L} \log \frac{1}{p_{Z^{L}}\left(Z^{L}\right)},
\end{aligned}
$$

where for a random sequence $\left\{W_{t}\right\}$,

$$
\begin{aligned}
& p-\liminf _{t \rightarrow \infty} W_{t}=\sup \left\{\beta \mid \lim _{t \rightarrow \infty} \operatorname{Pr}\left[W_{t}<\beta\right]=0\right\}, \\
& p-\limsup _{t \rightarrow \infty} W_{t}=\inf \left\{\beta \mid \lim _{t \rightarrow \infty} \operatorname{Pr}\left[W_{t}>\beta\right]=0\right\} .
\end{aligned}
$$

Remark 5. If $Z^{L}=\left(Z_{1}, Z_{2}, \ldots, Z_{L}\right)$ is a concatenation of two sequences of i.i.d. random variables on $\mathcal{Z}$ with distribution

$$
p_{Z^{L}}\left(z^{L}\right)=\prod_{i=1}^{\lceil\gamma L\rceil} p^{(1)}\left(z_{i}\right) \prod_{i=\lceil\gamma L\rceil+1}^{L} p^{(2)}\left(z_{i}\right),
$$

where $\lceil a\rceil$ is the smallest integer greater than or equal to $a$, then, we have

$$
\begin{aligned}
\lim _{L \rightarrow \infty} \frac{1}{L} \log \frac{1}{p_{Z^{L}}\left(Z^{L}\right)}=\lim _{L \rightarrow \infty} \frac{1}{L}\left(\sum_{i=1}^{\lceil\gamma L\rceil} \log \frac{1}{p^{(1)}\left(Z_{i}\right)}+\sum_{i=\lceil\gamma L\rceil+1}^{L} \log \frac{1}{p^{(2)}\left(Z_{i}\right)}\right) & \\
=\gamma \sum_{z \in \mathcal{Z}} p^{(1)}(z) \log \frac{1}{p^{(1)}(z)}+(1-\gamma) \sum_{z \in \mathcal{Z}} p^{(2)}(z) \log \frac{1}{p^{(2)}(z)} & \text { with probability 1 } \\
=\gamma H\left(p^{(1)}\right)+(1-\gamma) H\left(p^{(2)}\right) & \text { with probability 1, }
\end{aligned}
$$

where the first equality results from the independence of the random variables $Z_{1}, Z_{2}, \ldots$ and the second equality follows from the weak law of large numbers. Thus

$$
\begin{aligned}
& p-\liminf _{L \rightarrow \infty} \frac{1}{L} \log \frac{1}{p_{Z^{L}}\left(Z^{L}\right)}=\gamma H\left(p^{(1)}\right)+(1-\gamma) H\left(p^{(2)}\right), \\
& p-\limsup _{L \rightarrow \infty} \frac{1}{L} \log \frac{1}{p_{Z^{L}}\left(Z^{L}\right)}=\gamma H\left(p^{(1)}\right)+(1-\gamma) H\left(p^{(2)}\right),
\end{aligned}
$$

hence $\underline{H}(Z)=\bar{H}(Z)=\gamma H\left(p^{(1)}\right)+(1-\gamma) H\left(p^{(2)}\right)=\lim _{L \rightarrow \infty} 1 / L H\left(Z^{L}\right)$. 
Lemma 6 (Simulation of a source from another source). For each natural number L, consider an arbitrary distribution $p_{X^{L}}^{(L)}$ on sequences $\left(x_{1}, x_{2}, \ldots, x_{L}\right) \in \mathcal{X}^{L}$. Similarly, for each natural number $L$, consider an arbitrary distribution $q_{A^{L}}^{(L)}$ on sequences $\left(a_{1}, a_{2}, \ldots, a_{L}\right) \in$ $\mathcal{A}^{L}$. If $\underline{H}(X)>\bar{H}(A)$, then, for each natural number $L$, there exists a mapping $\varphi_{L}$ : $\mathcal{X}^{L} \rightarrow \mathcal{A}^{L}$ such that the total variation distance between the distributions of $\varphi_{L}\left(X^{L}\right)$ and $A^{L}$ vanishes asymptotically, i.e.,

$$
\lim _{L \rightarrow \infty}\left\|q_{A^{L}}^{(L)}-p_{\varphi_{L}\left(X^{L}\right)}^{(L)}\right\|_{T V}=0
$$

The proof of Lemma 6 can be found in $(\mathrm{Han}, 2003$, p. 110).

Remark 7. Observe that $\lim _{L \rightarrow \infty}\left\|p_{A^{L}}-p_{\varphi_{L}\left(X^{L}\right)}\right\|_{T V}=0$ in the above lemma implies that $\varphi_{L}\left(X^{L}\right)$ is almost statistically indistinguishable from $p_{A^{L}}$ for large values of $L$. In other words, there is no statistical test that can distinguish between $p_{A^{L}}$ and $p_{\varphi_{L}\left(X^{L}\right)}$ with a non-negligible probability.

Take some $\epsilon>0$. Since $\gamma H\left(p_{A}^{(1)}\right)+(1-\gamma) H\left(p_{A}^{(2)}\right)<H(X)$, Lemma 6 and Remark 5 imply that there exist mappings $\varphi_{L}: \mathcal{X}^{L} \rightarrow \mathcal{A}^{L}$ such that for large $L$, the pmf of $A^{L}=$ $\varphi_{L}\left(X^{L}\right)$ is approximated (in total variation distance) as

$$
\left\|p_{A^{L}}\left(a^{L}\right)-\prod_{t=1}^{\lceil\gamma L\rceil} p_{A}^{(1)}\left(a_{t}\right) \prod_{t=\lceil\gamma L\rceil+1}^{L} p_{A}^{(2)}\left(a_{t}\right)\right\|_{T V} \leq \epsilon .
$$

Therefore, by dividing the $T$ stages into $N$ blocks of $L$ stages, we construct a strategy $\sigma$ for Alice as follows: in each block (excluding the first block) Alice chooses action sequence $A_{c}^{L}=\varphi_{L}\left(X_{p}^{L}\right)$, where $X_{p}^{L}$ is the randomness source observed during the previous block; i.e., $A_{c}^{L}$ is for the current block, but $X_{p}^{L}$ is for the previous block.

The ideal distribution $\prod_{t=1}^{\lceil\gamma L\rceil} p_{A}^{(1)}\left(a_{t}\right) \prod_{t=\lceil\gamma L\rceil+1}^{L} p_{A}^{(2)}\left(a_{t}\right)$ gives Alice a payoff of

$$
\lceil\gamma L\rceil \min _{b \in \mathcal{B}} \mathbb{E}_{p_{A}^{(1)}}\left[u_{A, b}\right]+(L-\lceil\gamma L\rceil) \min _{b \in \mathcal{B}} \mathbb{E}_{p_{A}^{(2)}}\left[u_{A, b}\right]
$$

Alice's actual distribution is within $\epsilon$ total variation distance of the ideal distribution. By relating the total variation distance to the payoff differences, we obtain that difference 
between the payoff under the actual distribution and the ideal one is at most $\epsilon$ times $2 L \mathrm{M}$, where $\mathrm{M}$ is the maximum absolute value entry of the payoff table ( $L M$ is the maximum absolute value entry of the $L$ repetitions of the game). Thus, the distance between the average payoff of the block differs by at most $2 \epsilon \mathrm{M}$ from the average payoff under the ideal distribution. This completes the proof.

Remark 8. We constructed the strategy $\sigma$ for the case $T=N L$. In general, for $T=$ $N L+\delta$, where $\delta<L$, one can extend the first block to contain $L+\delta$ stages and choose $N$ large enough to diminish the effect of the first block on the average payoff.

\subsection{Generalized model: leakage of the randomness source}

We generalize the model of the repeated game of Section 3.1 and define the repeated game with leaked randomness source as follows: Let $X^{T}=\left(X_{1}, X_{2}, \ldots, X_{T}\right)$ and $Y^{T}=\left(Y_{1}, Y_{2}, \ldots, Y_{T}\right)$ be two sequences of random variables such that $\left(X_{t}, Y_{t}\right)$, for $t \in\{1,2, \ldots, T\}$, are drawn i.i.d. from a sample space $\mathcal{X} \times \mathcal{Y}$ with law $p_{X Y}$. In every stage $t \in\{1,2, \ldots, T\}$, Alice and Bob observe respective random sources $X_{t}$ and $Y_{t}$ privately and choose actions $A_{t} \in \mathcal{A}$ and $B_{t} \in \mathcal{B}$. Thus the only difference of this model with the model of Section 3.1 is that in every stage $t$, Bob observes $Y_{t}$ which is related to the observation of Alice $X_{t}$; hence, the history of observations of Bob is $\mathrm{H}_{2}^{t}=$ $\left(Y_{1}, A_{1}, B_{1}, \ldots, Y_{t-1}, A_{t-1}, B_{t-1}, Y_{t}\right)$ and $B_{t}=\tau_{t}\left(\mathrm{H}_{2}^{t}\right)$, where $\tau_{t}:(\mathcal{A} \times \mathcal{B})^{t-1} \times \mathcal{Y}^{t} \rightarrow \mathcal{B}$. As before, the history of observations of Alice is $\mathrm{H}_{1}^{t}=\left(X_{1}, A_{1}, B_{1}, \ldots, X_{t-1}, A_{t-1}, B_{t-1}, X_{t}\right)$. Note that Alice does not have access to any private sources of randomness except $\mathrm{H}_{1}^{t}$ and hence, she has to use the deterministic function $\sigma_{t}(\cdot)$, while Bob can utilize the random function $\tau_{t}(\cdot)$. Alice (respectively Bob) wishes to maximize (respectively minimize) the expected average payoff $\lambda_{T}(\sigma, \tau)$ defined in Equation (1). Note that if we set $Y_{t}$ to be constant random variables, the above repeated zero-sum game reduces to the one considered in Section 3.1.

The maxmin value of the repeated game with leaked randomness source is defined as in Definition 2 and characterized as follows: 
Theorem 9. The maxmin value of the repeated game with leaked randomness source defined in Section 3.3 is $\mathcal{J}_{\text {cav }}(H(X \mid Y))$, where $\mathcal{J}_{\text {cav }}($.$) is defined as in Theorem 3$.

To prove Theorem 9, in Section 3.3.1, we show that Alice can secure $\mathcal{J}_{\text {cav }}(H(X \mid Y))$ and in Section 3.3.2, we show that Bob can defend $\mathcal{J}_{\text {cav }}(H(X \mid Y))$. Therefore, by definition, $\mathcal{J}_{\text {cav }}(H(X \mid Y))$ is the maxmin value of the repeated game. Before going to Sections 3.3.1 and 3.3 .2 , we present a corollary followed by an example.

Corollary 10. Consider the case in which besides the sequence $X_{1}, X_{2}, \ldots$, Alice also observes the sequence $Y_{1}, Y_{2}, \ldots$; therefore, $X_{t}^{\prime}=\left(X_{t}, Y_{t}\right)$ is the observation of Alice at stage t. Since $H\left(X^{\prime} \mid Y\right)=H(X \mid Y)$, the maxmin value of the game is still $\mathcal{J}_{\text {cav }}(H(X \mid Y))$ which is equal to the maxmin value of the game in which Alice does not observe the sequence $Y_{1}, Y_{2}, \ldots$ Therefore, Alice's access to $Y_{1}, Y_{2}, \ldots$ does not help her. This parallels the classical result of Slepian and Wolf (1973) in information theory.

Example 11. (Matching pennies) Consider the matching pennies game with $\mathcal{A}=\mathcal{B}=$ $\{0,1\}$ and payoffs:

$$
u_{0,0}=u_{1,1}=1, \quad u_{0,1}=u_{1,0}=0 .
$$

Assume that Alice observes (symbol by symbol) an i.i.d. sequence of binary random variables $X_{1}, X_{2}, \ldots$, where

$$
p_{X_{i}}(0)=q, \quad p_{X_{i}}(1)=1-q .
$$

Each $X_{i}$ is revealed to Bob with probability $\alpha \in[0,1]$. Thus, Bob observes (symbol by symbol) a sequence $Y_{1}, Y_{2}, \ldots$ such that with probability $\alpha, Y_{i}=X_{i}$ and with probability $1-\alpha, Y_{i}=$ null.

Roughly speaking, a fraction of the bits of Alice are revealed to Bob. If Alice is made aware of which of her bits are compromised and leaked to Bob, she can drop the compromised bits and keep the $1-\alpha$ fraction of the secret bits. In this way, she can distill an average of $(1-\alpha) H(q)$ random bits per observation. It is known from Gossner and Vieille (2002) that for the matching pennies game, $\mathcal{J}_{\text {cav }}(\mathrm{h})=\mathrm{h} / 2$. Thus, using $(1-\alpha) H(q)$ bits 
per stage, Alice can secure payoff $(1-\alpha) H(q) / 2$. The above theorem says that Alice can secure the same payoff even without knowing which of her bits are compromised.

\subsubsection{Proof of Theorem 9: Alice can secure $\mathcal{J}_{\text {cav }}(H(X \mid Y))$}

To show that Alice can secure any payoff less than $\mathcal{J}_{\text {cav }}(H(X \mid Y))$, we extend and simplify the proof of Gossner and Vieille (2002). As before, the upper concave envelope $\mathcal{J}_{\text {cav }}(\cdot)$ at $\mathrm{h}=H(X \mid Y)$ can be expressed as the convex combination

$$
\gamma \mathcal{J}\left(H\left(p_{A}^{(1)}\right)\right)+(1-\gamma) \mathcal{J}\left(H\left(p_{A}^{(2)}\right)\right)
$$

for some $\gamma \in[0,1]$ and $\operatorname{pmfs} p_{A}^{(1)}$ and $p_{A}^{(2)}$ in $\Delta(\mathcal{A})$ where $p_{A}^{(1)}$ and $p_{A}^{(2)}$ secure respective payoffs $\mathcal{J}\left(H\left(p_{A}^{(1)}\right)\right)$ and $\mathcal{J}\left(H\left(p_{A}^{(2)}\right)\right)$ in the one-shot game, and the following equality is satisfied:

$$
\gamma H\left(p_{A}^{(1)}\right)+(1-\gamma) H\left(p_{A}^{(2)}\right)=H(X \mid Y)
$$

As a result, it suffices to show that for any $p_{A}^{(1)}$ and $p_{A}^{(2)}$ satisfying

$$
\gamma H\left(p_{A}^{(1)}\right)+(1-\gamma) H\left(p_{A}^{(2)}\right)<H(X \mid Y)
$$

Alice can secure payoffs arbitrarily close to

$$
\gamma \min _{b \in \mathcal{B}} \mathbb{E}_{p_{A}^{(1)}}\left[u_{A, b}\right]+(1-\gamma) \min _{b \in \mathcal{B}} \mathbb{E}_{p_{A}^{(2)}}\left[u_{A, b}\right]
$$

Again, the idea is to utilize the block-Markov proof technique. Take some $T$ of the form $T=N L$, and divide the total $T$ stages into $N$ blocks of length $L$. Each block is divided into two subblocks as before. Excluding the first block, Alice wants to play almost i.i.d. according to $p_{A}^{(1)}$ during the first subblock, and almost i.i.d. according to $p_{A}^{(2)}$ during the second subblock.

By symmetry, we only need to consider the payoff that Alice gets in one of the blocks. For notational simplicity, we denote the observations of Alice and Bob in the previous block by $X_{p}^{L}$ and $Y_{p}^{L}$ respectively, and use $A_{c}^{L}$ and $B_{c}^{L}$ to denote their actions in the current block. Instead of Lemma 6 in the previous section, the proof relies on the following proposition whose proof is given in Section 6.1 
Proposition 12. Let $\left(X_{p}^{L}, Y_{p}^{L}\right)$ be drawn i.i.d. from $p_{X Y}\left(x_{p}, y_{p}\right), \gamma \in[0,1]$ be an arbitrary real number, and $p_{A}^{(1)}$ and $p_{A}^{(2)}$ be arbitrary distributions on $\mathcal{A}$ such that

$$
\gamma H\left(p_{A}^{(1)}\right)+(1-\gamma) H\left(p_{A}^{(2)}\right)<H(X \mid Y)
$$

Then, for any $\epsilon>0$, there exists a natural number $\tilde{L}$ and mappings $\psi_{L}: \mathcal{X}^{L} \rightarrow \mathcal{A}^{L}$ such that for all $L \geq \tilde{L}$ and $A_{c}^{L}=\psi_{L}\left(X_{p}^{L}\right)$,

$$
\left\|p_{A_{c}^{L}, Y_{p}^{L}}\left(a_{c}^{L}, y_{p}^{L}\right)-p_{Y_{p}^{L}}\left(y_{p}^{L}\right) \prod_{t=1}^{\lceil\gamma L\rceil} p_{A}^{(1)}\left(a_{c, t}\right) \prod_{t=\lceil\gamma L\rceil+1}^{L} p_{A}^{(2)}\left(a_{c, t}\right)\right\|_{T V}<\epsilon .
$$

Note that $p_{Y_{p}^{L}}\left(y_{p}^{L}\right) \prod_{t=1}^{\lceil\gamma L\rceil} p_{A}^{(1)}\left(a_{c, t}\right) \prod_{t=\lceil\gamma L\rceil+1}^{L} p_{A}^{(2)}\left(a_{c, t}\right)$ is the distribution of the ideal independent actions desired by Alice, whereas $p_{A_{c}^{L}, Y_{p}^{L}}\left(a_{c}^{L}, y_{p}^{L}\right)$ is the real joint distribution.

Let Alice use the actions $A_{c}^{L}=\left(A_{c, 1}, A_{c, 2}, \ldots, A_{c, L}\right)$ in the current block, where $A_{c}^{L}=$ $\psi_{L}\left(X_{p}^{L}\right)$ and $\psi_{L}$ is the mapping of Proposition 12 . Because the $X$-source is i.i.d., the $X$-source for different blocks are independent, and random variable $A_{c}^{L}$ (a function of $\left.X_{p}^{L}\right)$ is independent of Alice's action in all the previous blocks. As a result, even though Bob has access to the entire past history of the game and his $Y$-source observations, he obtains information about $X_{p}^{L}$ only through his source $Y_{p}^{L}$ and Alice's prior actions in the current block. In other words, $B_{c, t}$, Bob's action at the $t$-th stage of the current block, is conditionally independent of $X_{p}^{L}$ given $Y_{p}^{L}, A_{c}^{t-1}, B_{c}^{t-1}$. Since $A_{c}^{L}=\psi_{L}\left(X_{p}^{L}\right), B_{c, t}$ is also conditionally independent of $A_{c, t}, A_{c, t+1}, \cdots, A_{c, L}$ given $Y_{p}^{L}, A_{c}^{t-1}, B_{c}^{t-1}$. Thus,

$$
p\left(b_{c}^{L} \mid y_{p}^{L}, a_{c}^{L}\right)=\prod_{t=1}^{L} p\left(b_{c, t} \mid y_{p}^{L}, a_{c}^{t-1}, b_{c}^{t-1}\right) .
$$

Then, utilizing the first property of total variation in Lemma 1 for random variables $E=\left(A_{c}^{L}, Y_{p}^{L}\right)$ and $F=B_{c}^{L}$ we conclude from (4) that

$$
\begin{aligned}
& \| p_{A_{c}^{L}, Y_{p}^{L}}\left(a_{c}^{L}, y_{p}^{L}\right) \prod_{t=1}^{L} p\left(b_{c, t} \mid y_{p}^{L}, a_{c}^{t-1}, b_{c}^{t-1}\right) \\
& \quad-p_{Y_{p}^{L}}\left(y_{p}^{L}\right) \prod_{t=1}^{\lceil\gamma L\rceil} p_{A}^{(1)}\left(a_{c, t}\right) p\left(b_{c, t} \mid y_{p}^{L}, a_{c}^{t-1}, b_{c}^{t-1}\right) \prod_{t=\lceil\gamma L\rceil+1}^{L} p_{A}^{(2)}\left(a_{c, t}\right) p\left(b_{c, t} \mid y_{p}^{L}, a_{c}^{t-1}, b_{c}^{t-1}\right) \|_{T V}<\epsilon .
\end{aligned}
$$


Utilizing the second property of total variation in Lemma 1 for random variables $E=$ $\left(A_{c}^{L}, B_{c}^{L}\right)$ and $F=Y_{p}^{L}$, we conclude that the distance between the actual actions $p_{A_{c}^{L}, B_{c}^{L}}\left(a_{c}^{L}, b_{c}^{L}\right)$ and the ideal one is less than or equal to $\epsilon$, i.e.,

$$
\left\|p_{A_{c}^{L}, B_{c}^{L}}\left(a_{c}^{L}, b_{c}^{L}\right)-\prod_{t=1}^{\lceil\gamma L\rceil} p_{A}^{(1)}\left(a_{c, t}\right) p\left(b_{c, t} \mid a_{c}^{t-1}, b_{c}^{t-1}\right) \prod_{t=\lceil\gamma L\rceil+1}^{L} p_{A}^{(2)}\left(a_{c, t}\right) p\left(b_{c, t} \mid a_{c}^{t-1}, b_{c}^{t-1}\right)\right\|_{T V}<\epsilon .
$$

As before, by relating the total variation distance to the payoff differences, we obtain that the average payoff differs by at most $2 \epsilon \mathrm{M}$ from the average payoff under the ideal distribution. This completes the proof.

\subsubsection{Proof of Theorem 9: Bob can defend $\mathcal{J}_{\text {cav }}(H(X \mid Y))$}

This is an extension of the proof given in Neyman and Okada (2000); Gossner and Vieille (2002). Let $\sigma$ be an arbitrary strategy for Alice and generate strategy $\tau$ for Bob as follows: given $h_{2}^{t}$, an arbitrary history of observations of Bob until stage $t, \tau_{t}\left(h_{2}^{t}\right)$ is the best choice of Bob that minimizes the expected payoff at stage $t$, i.e.,

$$
\tau_{t}\left(h_{2}^{t}\right) \in \underset{b \in \mathcal{B}}{\arg \min } \mathbb{E}_{\sigma}\left[u_{A_{t}, b} \mid \mathrm{H}_{2}^{t}=h_{2}^{t}\right],
$$

where $\mathbb{E}_{\sigma}$ denotes the expectation with respect to the probability distribution induced by $\sigma$ and i.i.d. repetitions of $p_{X, Y}$. Note that conditional on the observation $h_{2}^{t}$ of Bob, Alice's action $A_{t}$ has entropy $H\left(A_{t} \mid \mathrm{H}_{2}^{t}=h_{2}^{t}\right)$, thus,

$$
\mathbb{E}_{\sigma, \tau}\left[u_{A_{t}, B_{t}} \mid \mathbf{H}_{2}^{t}=h_{2}^{t}\right] \leq \mathcal{J}\left(H\left(A_{t} \mid \mathbf{H}_{2}^{t}=h_{2}^{t}\right)\right) \leq \mathcal{J}_{\text {cav }}\left(H\left(A_{t} \mid \mathbf{H}_{2}^{t}=h_{2}^{t}\right)\right) \text {. }
$$

Therefore, we have

$$
\begin{aligned}
\mathbb{E}_{\sigma, \tau}\left[u_{A_{t}, B_{t}}\right] & =\sum_{h_{2}^{t} \in(\mathcal{A} \times \mathcal{B})^{t-1} \times \mathcal{Y}^{t}} \operatorname{Pr}\left[\mathrm{H}_{2}^{t}=h_{2}^{t}\right] \mathbb{E}_{\sigma, \tau}\left[u_{A_{t}, B_{t}} \mid \mathrm{H}_{2}^{t}=h_{2}^{t}\right] \\
& \leq \sum_{h_{2}^{t} \in(\mathcal{A} \times \mathcal{B})^{t-1} \times \mathcal{Y}^{t}} \operatorname{Pr}\left[\mathrm{H}_{2}^{t}=h_{2}^{t}\right] \mathcal{J}_{\text {cav }}\left(H\left(A_{t} \mid \mathrm{H}_{2}^{t}=h_{2}^{t}\right)\right) \\
& \leq \mathcal{J}_{\text {cav }}\left(\sum_{h_{2}^{t} \in(\mathcal{A} \times \mathcal{B})^{t-1} \times \mathcal{Y}^{t}} \operatorname{Pr}\left[\mathrm{H}_{2}^{t}=h_{2}^{t}\right] H\left(A_{t} \mid \mathrm{H}_{2}^{t}=h_{2}^{t}\right)\right) \\
& =\mathcal{J}_{\text {cav }}\left(H\left(A_{t} \mid \mathrm{H}_{2}^{t}\right)\right),
\end{aligned}
$$


where the second inequality is implied by applying Jensen's inequality to concave function $\mathcal{J}_{\text {cav }}(\cdot)$. By definition of $\lambda_{T}(\sigma, \tau)$ and using (8) we have

$$
\begin{aligned}
\lambda_{T}(\sigma, \tau) & \leq \sum_{t=1}^{T} \frac{1}{T} \mathcal{J}_{\text {cav }}\left(H\left(A_{t} \mid \mathrm{H}_{2}^{t}\right)\right) \\
& \leq \mathcal{J}_{\text {cav }}\left(\sum_{t=1}^{T} \frac{1}{T} H\left(A_{t} \mid \mathrm{H}_{2}^{t}\right)\right) \\
& =\mathcal{J}_{\text {cav }}\left(\sum_{t=1}^{T} \frac{1}{T} H\left(A_{t} \mid Y^{t}, A^{t-1}, B^{t-1}\right)\right) \\
& =\mathcal{J}_{\text {cav }}\left(\sum_{t=1}^{T} \frac{1}{T} H\left(A_{t} \mid Y^{T}, A^{t-1}\right)\right) \\
& =\mathcal{J}_{\text {cav }}\left(\frac{1}{T} H\left(A^{T} \mid Y^{T}\right)\right) \\
& \leq \mathcal{J}_{\text {cav }}\left(\frac{1}{T} H\left(X^{T} \mid Y^{T}\right)\right) \\
& =\mathcal{J}_{\text {cav }}(H(X \mid Y)),
\end{aligned}
$$

where (9) follows from applying Jensen's inequality to concave function $\mathcal{J}_{\text {cav }}$. Note that given the strategy $\tau$ for Bob, $B^{t-1}$ is a deterministic function of $Y^{t}$ and $A^{t-1}$, thus $H\left(A_{t} \mid Y^{t}, A^{t-1}, B^{t-1}\right)=H\left(A_{t} \mid Y^{t}, A^{t-1}\right)$; furthermore, $A_{t}$ and $B_{t}$ are causally generated from i.i.d. sequences $X^{T}$ and $Y^{T}$, so $\left(A_{t}, A^{t-1}, Y^{t}\right)$ is independent of $\left(Y_{t+1}, Y_{t+2}, \ldots, Y_{T}\right)$ and hence $H\left(A_{t} \mid Y^{t}, A^{t-1}\right)=H\left(A_{t} \mid Y^{T}, A^{t-1}\right)$, which implies (10). To prove (11), consider that given strategies of the players and the sequence $Y^{T}, A^{T}$ is a deterministic function of $X^{T}$ and hence $H\left(X^{T} \mid Y^{T}\right) \geq H\left(A^{T} \mid Y^{T}\right)$; therefore, since $\mathcal{J}_{\text {cav }}(\cdot)$ is an increasing function, (11) follows.

Thus, for all strategy $\sigma$ of Alice there exists a strategy $\tau$ for Bob such that $\lim \sup _{T \rightarrow \infty} \lambda_{T}(\sigma, \tau) \leq$ $\mathcal{J}_{\text {cav }}(H(X \mid Y))$. Hence, Bob can defend $\mathcal{J}_{\text {cav }}(H(X \mid Y))$.

\section{Secret correlation in repeated games with imperfect monitoring}

In this section, we revisit the repeated game with imperfect monitoring studied by Gossner and Tomala (2007). The main contribution of this part is to simplify the proof 
of Gossner and Tomala (2007) and generalize their results.

\subsection{Problem definition}

Consider a $T$ stage repeated zero-sum game between team $A$ and player $B$, where team $A$ consists of $m$ players $\{1,2, \ldots, m\}$ with respective finite actions sets $\mathcal{A}_{1}, \mathcal{A}_{2}, \ldots, \mathcal{A}_{m}$. Let $\mathcal{A}=\mathcal{A}_{1} \times \mathcal{A}_{2} \times \cdots \times \mathcal{A}_{m}$ and $\mathcal{B}$ denote the finite actions sets of team $A$ and player $B$, respectively. In every stage $t \in\{1,2, \ldots, T\}$, all players choose an action from their corresponding sets of actions. Let $\mathbf{A}_{t}$ denote the action profile of team $A$ and $B_{t}$ denote the action of player $B$ at stage $t$. At the end of stage $t$, team $A$ gets payoff $u_{\mathbf{A}_{t}, B_{t}}$ from player $B$ and all players of team $A$ observe the chosen actions $\mathbf{A}_{t}$ and $B_{t}$, while player $B$ observes $B_{t}$ and $S_{t} \in \mathcal{S}$, where $S_{t}$ is a noisy version of $\mathbf{A}_{t}$ with conditional distribution $\operatorname{Pr}\left[S_{t}=s_{t} \mid \mathbf{A}_{t}=\mathbf{a}_{t}\right]=p_{S \mid \mathbf{A}}\left(s_{t} \mid \mathbf{a}_{t}\right)$. In order to choose the actions of stage $t$, players make use of the history of their observations until stage $t$, which is denoted by $\mathrm{H}_{1}^{t}=\left(\mathbf{A}^{t-1}, B^{t-1}\right)$ for players of team $A$ and $\mathrm{H}_{2}^{t}=\left(B^{t-1}, S^{t-1}\right)$ for player $B$. Let $\sigma_{i, t}:(\mathcal{A} \times \mathcal{B})^{t-1} \rightarrow \mathcal{A}_{i}$ and $\tau_{t}:(\mathcal{B} \times \mathcal{S})^{t-1} \rightarrow \mathcal{B}$ be the random functions mapping the history of observations of arbitrary player $i$ in team $A$ and player $B$ to their actions at stage $t$, thus, $\mathbf{A}_{t}=$ $\left(\sigma_{1, t}\left(\mathbf{H}_{1}^{t}\right), \sigma_{2, t}\left(\mathbf{H}_{1}^{t}\right), \ldots, \sigma_{m, t}\left(\mathbf{H}_{1}^{t}\right)\right)$ and $B_{t}=\tau_{t}\left(\mathbf{H}_{2}^{t}\right)$. Let $\sigma_{t}=\left(\sigma_{1, t}, \sigma_{2, t}, \ldots, \sigma_{m, t}\right)$. We call the $T$-tuples $\sigma=\left(\sigma_{1}, \sigma_{2}, \ldots, \sigma_{T}\right)$ and $\tau=\left(\tau_{1}, \tau_{2}, \ldots, \tau_{T}\right)$ the strategies of team $A$ and player $B$, respectively. The expected average payoff for team $A, \lambda_{T}(\sigma, \tau)$, and the maxmin value of the game are defined in a similar way as in Section 3.1 .

Remark 13. In the definition of the repeated game with imperfect monitoring, Gossner and Tomala (2007) assumed that the signals $S_{t}$ were also observed by the players of team $A$, whereas we assume that the signals $S_{t}$ are not observed by the players of team $A$. We generalize the result of Gossner and Tomala (2007) by showing that the maxmin value of the game remains the same with this change in assumption.

Definition 14. Let $\Pi$ be a subset of $\Delta(\mathcal{A})$ containing the distributions of independent random actions on $\mathcal{A}=\mathcal{A}_{1} \times \mathcal{A}_{2} \times \cdots \times \mathcal{A}_{m}$, i.e.,

$$
\Pi=\left\{p_{\mathbf{A}}=p_{A_{1}} p_{A_{2}} \ldots p_{A_{m}} \mid p_{A_{i}} \in \Delta\left(\mathcal{A}_{i}\right), \forall i=1, \ldots, m\right\}
$$


Furthermore, given random variables $R \in \mathcal{R}$ and $\mathbf{A} \in \mathcal{A}$ with joint distribution $p_{\mathbf{A} R}$, functions $\pi(\mathbf{A} \mid R=r)$ (the security level of $\mathbf{A}$ given $R=r$ ) and $\pi(\mathbf{A} \mid R)$ (the security level of $\mathbf{A}$ given $R$ ) are defined as follows:

$$
\pi(\mathbf{A} \mid R=r)=\min _{b \in \mathcal{B}} \mathbb{E}\left[u_{\mathbf{A}, b} \mid R=r\right]
$$

and

$$
\pi(\mathbf{A} \mid R)=\sum_{r \in \mathcal{R}} p_{R}(r) \pi(\mathbf{A} \mid R=r) .
$$

Observe that $\pi(\mathbf{A} \mid R)$ is in terms of an average over values of $R$ and we have chosen the notation $\pi(\mathbf{A} \mid R)$ as its definition resembles the way conditional entropy is defined in information theory.

Theorem 15. The maxmin value of the repeated game with imperfect monitoring is

$$
w=\max \pi(\mathbf{A} \mid R)
$$

where the maximization is over all random variables $\mathbf{A} \in \mathcal{A}, S \in \mathcal{S}, R \in \mathcal{R}=\{0,1\}$ and $Q \in \mathcal{Q}=\{1,2,3, \cdots, 2|\mathcal{A}|\}$, satisfying

$$
\begin{aligned}
p_{S \mathbf{A} R Q}(s, \mathbf{a}, r, q) & =p_{R Q}(r, q) p_{\mathbf{A} \mid Q}(\mathbf{a} \mid q) p_{S \mid \mathbf{A}}(s \mid \mathbf{a}), \\
p_{\mathbf{A} \mid Q}(\mathbf{a} \mid q) & \in \Pi, \\
H(Q \mathbf{A} \mid S R) & \geq H(Q \mid R) .
\end{aligned}
$$

The set $\Pi$ and the function $\pi(\mathbf{A} \mid R)$ are defined in Definition 14 and $p_{S \mid \mathbf{A}}$ is the fixed conditional distribution that generates the monitoring signals $S_{t}$ of the repeated game with imperfect monitoring.

Remark 16. The statement of the above theorem has a different (but equivalent) form than the one given in Gossner and Tomala (2007). In particular, Gossner and Tomala (2007) have expressed the solution as an optimization problem over the set of "distributions of distributions". The computational aspects of the solution given by Gossner and Tomala 
(2007) has been studied by Gossner et al. (2009). In our characterization, because all the variables have finite alphabet sets, the above form is computable and is expressed as a maximization problem over a compact and bounded set of probability distribution.

In Section 4.2, we show that team $A$ can secure $w$, and in Section 4.3 , we show that player $B$ can defend $w$ even when players of team $A$, in addition to $\mathbf{A}_{t}$ and $B_{t}$, observe the signals $S_{t}$. Thus, $w$ is the maxmin value of the repeated game with imperfect monitoring regardless of whether team $A$ observe $S_{t}$ or not.

\subsection{Proof of Theorem 15: Payoff secured by team A}

We begin with investigating the special case in which player $B$ perfectly monitors the actions of team $A$; i.e., there exists a deterministic function $f: \mathcal{S} \rightarrow \mathcal{A}$ such that for all stages $t$, we have $\mathbf{A}_{t}=f\left(S_{t}\right)$. Let $\mathbf{A}, S, R, Q$ be arbitrary random variables with joint $\operatorname{pmf} p_{R Q \mathbf{A} S}$ in the feasible set of the maximization in the statement of the theorem. Considering that $\mathbf{A}=f(S)$, we have:

$$
H(Q \mathbf{A} \mid R S)-H(Q \mid R)=H(Q \mid R S)-H(Q \mid R) \geq 0,
$$

where the inequality follows from (14). On the other hand, we have $H(Q \mid R S) \leq H(Q \mid R)$. Hence, $H(Q \mid R S)=H(Q \mid R)$. Since $H(Q \mid R S)-H(Q \mid R)=H(S \mid R Q)-H(S \mid R)$, we obtain that $H(S \mid R Q)=H(S \mid R)$. It is known that $H(S \mid R Q) \leq H(S \mid R)$ (conditioning reduces entropy), and equality $H(S \mid R Q)=H(S \mid R)$ holds only if for all $r \in \mathcal{R}$ and $q \in \mathcal{Q}$ such that $p_{R Q}(r, q)>0$, we have $p_{S \mid R=r, Q=q}=p_{S \mid R=r}$. Since $\mathbf{A}$ is a deterministic function of $S$, we also conclude that for all $r \in \mathcal{R}$ and $q \in \mathcal{Q}$ such that $p_{R Q}(r, q)>0$, we have $p_{\mathbf{A} \mid R=r, Q=q}=p_{\mathbf{A} \mid R=r}$. On the other hand, Equations (12) and (13) imply that $p_{\mathbf{A} \mid R=r, Q=q}=p_{\mathbf{A} \mid Q=q} \in \Pi$; thus, for all $r$ with positive probability, we have $p_{\mathbf{A} \mid R=r} \in \Pi$.

Let $T$ be the total number of the stages of the game. Construct a strategy $\sigma$ for team $A$ as follows: in the first $\left\lceil p_{R}(0) T\right\rceil$ stages, the team players play i.i.d. according to $p_{\mathbf{A} \mid R=0}$, and in the remaining $T-\left\lceil p_{R}(0) T\right\rceil$ stages, they play i.i.d. according to $p_{\mathbf{A} \mid R=1}$. Note that since for all $r$ with positive probability, $p_{\mathbf{A} \mid R=r}$ belongs to $\Pi$, the team players 
can implement the above strategy distributively without the need for shared randomness. When $T$ tends to infinity, the expected average payoff secured by $\sigma$ converges to $\pi(\mathbf{A} \mid R)$, and hence, they can secure $\pi(\mathbf{A} \mid R)$.

Next, we assume that player $B$ monitors the actions of the team players imperfectly, i.e., Bob is unable to compute $\mathbf{A}$ as a deterministic function of $S$. Let $\mathbf{A} \in \mathcal{A}, S \in \mathcal{S}$, $R \in \mathcal{R}=\{0,1\}$ and $Q \in \mathcal{Q}=\{1,2,3, \cdots, 2|\mathcal{A}|\}$ be arbitrary random variables with joint pmf $p_{R Q \mathbf{A} S}$ satisfying (12), (13) and the strict form of (14), i.e.,

$$
H(Q \mathbf{A} \mid R S)>H(Q \mid R)
$$

The boundary case, $H(Q \mathbf{A} \mid R S)=H(Q \mid R)$, will be addressed in Remark 19. We now show that team $A$ can secure payoff $\pi(\mathbf{A} \mid R)$. Since

$$
\pi(\mathbf{A} \mid R)=p_{R}(0) \min _{b \in \mathcal{B}} \mathbb{E}\left[u_{\mathbf{A}, b} \mid R=0\right]+p_{R}(1) \min _{b \in \mathcal{B}} \mathbb{E}\left[u_{\mathbf{A}, b} \mid R=1\right],
$$

it suffices to show that players of team $A$ can choose their actions almost i.i.d. according to $p_{\mathbf{A} \mid R}(\mathbf{a} \mid 0)$ in $\gamma=p_{R}(0)$ portion of stages and almost i.i.d according to $p_{\mathbf{A} \mid R}(\mathbf{a} \mid 1)$ in $1-\gamma=p_{R}(1)$ portion of stages, while the action of each stage is almost independent of the history of observations of player $B$ until that stage.

In the rest of the proof, the ideal joint distribution $p_{R Q \mathbf{A} S}$ is assumed to be given and fixed. Random variable $\mathbf{A}$ should not be confused with $\mathbf{A}_{1}$ or $\mathbf{A}^{T}=\left(\mathbf{A}_{1}, \mathbf{A}_{2}, \cdots, \mathbf{A}_{T}\right)$ which denote the action profile of players of team $A$ at the first stage and the $T$ time instances, respectively.

The block-Markov technique: As in the previous section, team $A$ utilizes the block-Markov strategy. We assume that the game is played over one block of length $k L$ (the first block) followed by $N$ blocks of length $L$. Therefore, the total number of repetitions of the game is $T=k L+N L$. The first block is of length $k L$, which will be specified later; this block is sufficiently long block to provide enough randomness to initialize the block-Markov strategy for the $N$ blocks of length $L$. Excluding the first block, each block is divided into two subblocks of length $\lceil\gamma L\rceil$ and $L-\lceil\gamma L\rceil$. Team $A$ aims to play almost i.i.d. according to $p_{\mathbf{A} \mid R}(\mathbf{a} \mid 0)$ in the first subblock and according to 
$p_{\mathbf{A} \mid R}(\mathbf{a} \mid 1)$ in the second subblock, while the action of each stage is almost independent of the history of observations of player $B$ until that stage. To do so, in each block, the players of team $A$ extract a sequence of shared randomness, which is almost independent of the observations of player $B$, to correlate their actions in the next block. For arbitrary block $i \geq 3$ (the current block), let $Q_{c}^{L} \in \mathcal{Q}^{L}$ denote the sequence of shared randomness extracted in previous block and $\mathbf{A}_{c}^{L}$ denote the sequence of actions of team $A$ played in the current block. Players of team $A$ produce their actions in the current block, $\mathbf{A}_{c}^{L}$, only from the shared randomness $Q_{c}^{L}$ and their private randomness in the manner that is described in details below. With team $A$ playing $\mathbf{A}_{c, t}$ in the $t$-th stage of the current block, player $B$ gets the signal $S_{c, t}$ constructed from $\mathbf{A}_{c, t}$ with the conditional distribution $p_{S \mid \mathbf{A}}$.

For simplicity of the notation, the sequence of shared randomness, the actions of team $A$, the actions of player $B$ and the signals observed by player $B$ are denoted by $Q_{c}^{L}, \mathbf{A}_{c}^{L}, B_{c}^{L}, S_{c}^{L}$ for the current block ( $i$-th block) and by $Q_{p}^{L}, \mathbf{A}_{p}^{L}, B_{p}^{L}, S_{p}^{L}$ for the previous block ( $i-1)$-th block), where $i \geq 3$. Similarly, the sequence of shared randomness, the actions of team $A$, the actions of player $B$ and the signals observed by player $B$ in the second block are denoted by $Q_{s}^{L}, \mathbf{A}_{s}^{L}, B_{s}^{L}, S_{s}^{L}$. For the first block we use $\mathbf{A}_{f}^{k L}, B_{f}^{k L}$ and $S_{f}^{k L}$ to denote these random variables.

First block: In the first block, players of team $A$ start off without any shared randomness. They choose their actions independently and i.i.d. according to some $p_{\mathbf{A}}^{(0)} \in \Pi$ satisfying $H\left(\mathbf{A}^{(0)} \mid S^{(0)}\right)>0$, where $\left(\mathbf{A}^{(0)}, S^{(0)}\right)$ are some random variables with joint pmf $p_{\mathbf{A} S}^{(0)}(\mathbf{a}, s)=p_{\mathbf{A}}^{(0)}(\mathbf{a}) p_{S \mid \mathbf{A}}(s \mid \mathbf{a})$. Note that the distribution $p_{\mathbf{A}}^{(0)}$ with the above specifications exists because player $B$ does not have a perfect monitoring of the actions of the team players. Furthermore, since $p_{\mathbf{A}}^{(0)} \in \Pi$ and the players of team $A$ have access to private randomness, they can implement it distributively. Thus,

$$
p_{\mathbf{A}_{f}^{k L, S} S_{f}^{k L}}\left(\mathbf{a}_{f}^{k L}, s_{f}^{k L}\right)=\prod_{t=1}^{k L} p_{\mathbf{A}}^{(0)}\left(\mathbf{a}_{f, t}\right) p_{S \mid A}\left(s_{f, t} \mid \mathbf{a}_{f, t}\right) .
$$

The length of the first block is $k L$, where $L$ will be specified later; here $k$ is a natural 
number satisfying

$$
k>\frac{H(Q \mid R)}{H\left(\mathbf{A}^{(0)} \mid S^{(0)}\right)} .
$$

Second block: Equation (16) implies that $k H\left(\mathbf{A}^{(0)} \mid S^{(0)}\right)>H(Q \mid R)$. Therefore, according to Proposition 12 , for arbitrary $\epsilon>0$, there exist mappings $\varphi_{L}: \mathcal{A}^{k L} \rightarrow \mathcal{Q}^{L}$ such that if we take $Q_{s}^{L}=\varphi_{L}\left(\mathbf{A}_{f}^{k L}\right)$, then, for sufficiently large $L$ we have:

$$
\left\|p_{Q_{s}^{L}, S_{f}^{k L}}\left(q_{s}^{L}, s_{f}^{k L}\right)-p_{S_{f}^{k L}}\left(s_{f}^{k L}\right) \prod_{t=1}^{\lceil\gamma L\rceil} p_{Q \mid R}\left(q_{s, t} \mid 0\right) \prod_{t=\lceil\gamma L\rceil+1}^{L} p_{Q \mid R}\left(q_{s, t} \mid 1\right)\right\|_{T V} \leq \epsilon .
$$

This implies that each of the players of team $A$ can extract shared randomness $Q_{s}^{L}$ which is almost independent of the observation of player B in the first block. Let $\mathrm{H}_{2, s}=\left(S_{f}^{k L}, B_{f}^{k l}\right)$ denote the history of observations of player $B$ before starting the second block. Since $B_{f}^{k L}$ is produced locally by player $\mathrm{B}$ from $S_{f}^{k L}$, random variable $B_{f}^{k L}$ is conditionally independent of $Q_{s}^{L}$ given $S_{f}^{k L}$. Thus using the first property of total variation in Lemma 1 for random variables $E=\left(S_{f}^{k L}, Q_{s}^{L}\right)$ and $F=B_{f}^{k L}$ we have

$$
\left\|p_{Q_{s}^{L}, \mathrm{H}_{2, s}}\left(q_{s}^{L}, h_{2, s}\right)-p_{\mathrm{H}_{2, s}}\left(h_{2, s}\right) \prod_{t=1}^{\lceil\gamma L\rceil} p_{Q \mid R}\left(q_{s, t} \mid 0\right) \prod_{t=\lceil\gamma L\rceil+1}^{L} p_{Q \mid R}\left(q_{s, t} \mid 1\right)\right\|_{T V} \leq \epsilon .
$$

In the second block, the players of team $A$ (all of whom know $Q_{s}^{L}$ ) choose $\mathbf{A}_{s, t}$ with distribution $p_{\mathbf{A}_{s, t} \mid Q_{s, t}}=p_{\mathbf{A} \mid Q}$ from $\mathcal{A}$, that is

$$
p_{\mathbf{A}_{s}^{L} \mid Q_{s}^{L}, \mathrm{H}_{2, s}}\left(\mathbf{a}_{s}^{L} \mid q_{s}^{L}, h_{2, s}\right)=\prod_{t=1}^{L} p_{\mathbf{A} \mid Q}\left(\mathbf{a}_{s, t} \mid q_{s, t}\right) .
$$

Because $p_{\mathbf{A} \mid Q} \in \Pi$, actions of players of team $A$ are mutually independent given $Q_{s}^{L}$ and they can realize it using private randomness. Player $B$ gets signal $S_{s, t}$ constructed from $\mathbf{A}_{s, t}$ with the conditional distribution $p_{S \mid \mathbf{A}}$, i.e.,

$$
p_{S_{s}^{L} \mid \mathbf{A}_{s}^{L}, Q_{s}^{L}, \mathrm{H}_{2, s}}\left(s_{s}^{L} \mid \mathbf{a}_{s}^{L}, q_{s}^{L}, h_{2, s}\right)=\prod_{t=1}^{L} p_{S \mid \mathbf{A}}\left(s_{s, t} \mid \mathbf{a}_{s, t}\right) .
$$

In the $i$-th block for $i \geq 3$ : Let $Q_{p}^{L}, \mathbf{A}_{p}^{L}, B_{p}^{L}, S_{p}^{L}$ be the variables of the previous block, i.e., the $(i-1)$-th block. Note that $Q_{p}^{L}, \mathbf{A}_{p}^{L}$ is available to all players of team $A$. 
The idea is to construct the current block's shared randomness $Q_{c}^{L}$ from $\left(Q_{p}^{L}, \mathbf{A}_{p}^{L}\right)$ of the previous block. To do this, we need to find a suitable mapping $\psi_{L}: \mathcal{Q}^{L} \times \mathcal{A}^{L} \rightarrow \mathcal{Q}^{L}$ and set $Q_{c}^{L}=\psi_{L}\left(Q_{p}^{L}, \mathbf{A}_{p}^{L}\right)$. Once the shared randomness $Q_{c}^{L}$ is constructed in the current block, players of team $A$ construct their actions in the current block solely based on $Q_{c}^{L}$ using the conditional distribution $p_{\mathbf{A}_{c, t} \mid Q_{c, t}}=p_{\mathbf{A} \mid Q}$ as follows:

$$
p_{\mathbf{A}_{c}^{L} \mid Q_{c}^{L}}\left(\mathbf{a}_{c}^{L} \mid q_{c}^{L}\right)=\prod_{t=1}^{L} p_{\mathbf{A} \mid Q}\left(\mathbf{a}_{c, t} \mid q_{c, t}\right)
$$

In other words, while players of team $A$ observe the entire past history of the actions, their actions in stage $t$ of the current block depends only on $Q_{c, t}$. As before, because $p_{\mathbf{A} \mid Q} \in \Pi$, distributed implementation of this conditional distribution is feasible with the private randomness of the team players.

Considering (18) as the induction basis, suppose that in the previous block $((i-1)$-th block) we have

$$
\left\|p_{Q_{p}^{L}, \mathrm{H}_{2, p}}\left(q_{p}^{L}, h_{2, p}\right)-p_{\mathrm{H}_{2, p}}\left(h_{2, p}\right) \prod_{t=1}^{\lceil\gamma L\rceil} p_{Q \mid R}\left(q_{p, t} \mid 0\right) \prod_{t=\lceil\gamma L\rceil+1}^{L} p_{Q \mid R}\left(q_{p, t} \mid 1\right)\right\|_{T V} \leq 3^{(i-3)} \epsilon,
$$

where $\mathrm{H}_{2, p}$ is the observation of player $B$ before starting the previous block. Our first goal is to identify an appropriate mapping $\psi_{L}: \mathcal{Q}^{L} \times \mathcal{A}^{L} \rightarrow \mathcal{Q}^{L}$ to construct the shared randomness of current block $Q_{c}^{L}$ such that

$$
\left\|p_{Q_{c}^{L}, \mathrm{H}_{2, c}}\left(q_{c}^{L}, h_{2, c}\right)-p_{\mathrm{H}_{2, c}}\left(h_{2, c}\right) \prod_{t=1}^{\lceil\gamma L\rceil} p_{Q \mid R}\left(q_{c, t} \mid 0\right) \prod_{t=\lceil\gamma L\rceil+1}^{L} p_{Q \mid R}\left(q_{c, t} \mid 1\right)\right\|_{T V} \leq 3^{(i-2)} \epsilon
$$

where $\mathrm{H}_{2, c}=\left(\mathrm{H}_{2, p}, S_{p}^{L}, B_{p}^{L}\right)$ is the observation of player $B$ before starting the current block.

Random variables $\mathbf{A}_{p}^{L}$ and $S_{p}^{L}$ are produced in the previous block as follows:

$$
p_{\mathbf{A}_{p}^{L}, S_{p}^{L} \mid Q_{p}^{L}}\left(\mathbf{a}_{p}^{L}, s_{p}^{L} \mid q_{p}^{L}\right)=\prod_{t=1}^{L} p_{\mathbf{A} \mid Q}\left(\mathbf{a}_{p, t} \mid q_{p, t}\right) p_{S \mid \mathbf{A}}\left(s_{p, t} \mid \mathbf{a}_{p, t}\right) .
$$

Using the first property of total variation in Lemma 1 for $E=\left(Q_{p}^{L}, \mathrm{H}_{2, p}\right)$ and $F=\left(\mathbf{A}_{p}^{L}, S_{p}^{L}\right)$ 
along with Equation (19) we obtain

$$
\begin{aligned}
\| p_{Q_{p}^{L}, \mathbf{A}_{p}^{L}, S_{p}^{L}, \mathrm{H}_{2, p}} & \left(q_{p}^{L}, \mathbf{a}_{p}^{L}, s_{p}^{L}, h_{2, p}\right)-p_{\mathrm{H}_{2, p}}\left(h_{2, p}\right) \times \\
& \prod_{t=1}^{\lceil\gamma L\rceil} p_{Q \mathbf{A} S \mid R}\left(q_{p, t}, \mathbf{a}_{p, t}, s_{p, t} \mid 0\right) \prod_{t=\lceil\gamma L\rceil+1}^{L} p_{Q \mathbf{A} S \mid R}\left(q_{p, t}, \mathbf{a}_{p, t}, s_{p, t} \mid 1\right) \|_{T V} \leq 3^{(i-3)} \epsilon .
\end{aligned}
$$

We use the following proposition which is a generalization of Proposition 12 .

Proposition 17. Let $(U, X, Y) \in\{0,1\} \times \mathcal{X} \times \mathcal{Y}$ and $(V, Z) \in\{0,1\} \times \mathcal{Z}$ be arbitrary random variables with respective distributions $p_{U X Y}$ and $p_{V Z}$ with finite supports such that

$$
H(X \mid Y U)>H(Z \mid V)
$$

We also define random variables $\left(X_{1}, \cdots, X_{L}, Y_{1}, \cdots, Y_{L}, W\right)$ with the joint distribution $p_{X^{L} Y^{L} W}$ such that

$$
\left\|p_{X^{L} Y^{L} W}\left(x^{L}, y^{L}, w\right)-p_{W}(w) \prod_{t=1}^{\left\lceil p_{U}(0) L\right\rceil} p_{X Y \mid U}\left(x_{t}, y_{t} \mid 0\right) \prod_{t=\left\lceil p_{U}(0) L\right\rceil+1}^{L} p_{X Y \mid U}\left(x_{t}, y_{t} \mid 1\right)\right\|_{T V} \leq \delta_{1} .
$$

Then, for arbitrary $\delta_{2}>0$, there exist mappings $\psi_{L}: \mathcal{X}^{L} \rightarrow \mathcal{Z}^{L}$ and natural number $\bar{L}$ such that for all $L \geq \bar{L}$ and $Z^{L}=\psi_{L}\left(X^{L}\right)$ we have

$$
\left\|p_{Z^{L} Y^{L} W}\left(z^{L}, y^{L}, w\right)-p_{W}(w) p_{Y^{L}}\left(y^{L}\right) \prod_{t=1}^{\left\lceil p_{V}(0) L\right\rceil} p_{Z \mid V}\left(z_{t} \mid 0\right) \prod_{t=\left\lceil p_{V}(0) L\right\rceil+1}^{L} p_{Z \mid V}\left(z_{t} \mid 1\right)\right\|_{T V} \leq 2 \delta_{1}+\delta_{2} .
$$

Proof. The proof of Proposition 17 is provided in Section 6.2.

We use Proposition 17 with the choice of $X=(Q, \mathbf{A}), Y=S, U=R, Z=Q, V=R$, $X^{L}=\left(Q_{p}^{L}, \mathbf{A}_{p}^{L}\right), Y^{L}=S_{p}^{L}, Z^{L}=Q_{c}^{L}$ and $W=\mathrm{H}_{2, p}$. The assumptions of Proposition 17 are satisfied by Equations (15) and (21). Therefore, we obtain that for sufficiently large $L$, there exists a mapping $\psi_{L}: \mathcal{Q}^{L} \times \mathcal{A}^{L} \rightarrow \mathcal{Q}^{L}$ such that for $Q_{c}^{L}=\psi_{L}\left(Q_{p}^{L}, \mathbf{A}_{p}^{L}\right)$ we have

$$
\begin{gathered}
\left\|p_{Q_{c}^{L}, S_{p}^{L}, \mathrm{H}_{2, p}}\left(q_{c}^{L}, s_{p}^{L}, h_{2, p}\right)-p_{S_{p}^{L}}\left(s_{p}^{L}\right) p_{\mathrm{H}_{2, p}}\left(h_{2, p}\right) \prod_{t=1}^{\lceil\gamma L\rceil} p_{Q \mid R}\left(q_{c, t} \mid 0\right) \prod_{t=\lceil\gamma L\rceil+1}^{L} p_{Q \mid R}\left(q_{c, t} \mid 1\right)\right\|_{T V} \\
\leq 2 \times 3^{(i-3)} \epsilon+\epsilon \leq 3^{(i-2)} \epsilon .
\end{gathered}
$$


Since $B_{p}^{L}$ is conditionally independent of $Q_{c}^{L}$ given $\left(\mathrm{H}_{2, p}, S_{p}^{L}\right)$, using the first property of total variation in Lemma 1 for $E=\left(Q_{c}^{L}, S_{p}^{L}, \mathrm{H}_{2, p}\right)$ and $F=B_{p}^{L}$ we have

$$
\left\|p_{Q_{c}^{L}, \mathrm{H}_{2, c}}\left(q_{c}^{L}, h_{2, c}\right)-p_{\mathrm{H}_{2, c}}\left(h_{2, c}\right) \prod_{t=1}^{\lceil\gamma L\rceil} p_{Q \mid R}\left(q_{c, t} \mid 0\right) \prod_{t=\lceil\gamma L\rceil+1}^{L} p_{Q \mid R}\left(q_{c, t} \mid 1\right)\right\|_{T V} \leq 3^{(i-2)} \epsilon,
$$

where we utilized the notation $\mathrm{H}_{2, c}=\left(\mathrm{H}_{2, p}, S_{p}^{L}, B_{p}^{L}\right)$. Therefore, by induction, Equation (23) holds for arbitrary $i \geq 3$.

Calculation of the payoff of the $i$-th block for $i \geq 2$ : Actions of team $A$ and the signals $S_{c}^{L}$ were produced according to

$$
p_{\mathbf{A}_{c}^{L}, S_{c}^{L} \mid \mathrm{H}_{2, c}, Q_{c}^{L}}\left(\mathbf{a}_{c}^{L} \mid h_{2, c}, q_{c}^{L}\right)=\prod_{t=1}^{L} p_{\mathbf{A} \mid Q}\left(\mathbf{a}_{c, t} \mid q_{c, t}\right) p_{S \mid \mathbf{A}}\left(s_{c, t} \mid \mathbf{a}_{c, t}\right) .
$$

Player $B$ constructs his action $B_{c, t}$ from the observations available to him at stage $t$ of the current block, that is

$$
p_{B_{c}^{L} \mid \mathrm{H}_{2, c}, Q_{c}^{L}, \mathbf{A}_{c}^{L}, S_{c}^{L}}\left(b_{c}^{L} \mid h_{2, c}, q_{c}^{L}, \mathbf{a}_{c}^{L}, s_{c}^{L}\right)=\prod_{t=1}^{L} p_{B_{c, t} \mid \mathrm{H}_{2, c}, S_{c}^{t-1}, B_{c}^{t-1}}\left(b_{c, t} \mid h_{2, c}, s_{c}^{t-1}, b_{c}^{t-1}\right) .
$$

Therefore, using the first property of total variation in Lemma 1 for random variables $E=\left(\mathrm{H}_{2, c}, Q_{c}^{L}\right)$ and $F=\left(\mathbf{A}_{c}^{L}, B_{c}^{L}, S_{c}^{L}\right)$ along with Equation (23), we obtain

$$
\begin{aligned}
& \| p_{Q_{c}^{L}, \mathbf{A}_{c}^{L}, S_{c}^{L}, \mathrm{H}_{2, c}, B_{c}^{L}}\left(q_{c}^{L}, \mathbf{a}_{c}^{L}, s_{c}^{L}, h_{2, c}, b_{c}^{L}\right)-p_{\mathrm{H}_{2, c}}\left(h_{2, c}\right) \times \\
& \prod_{t=1}^{\lceil\gamma L\rceil} p_{Q \mid R}\left(q_{c, t} \mid 0\right) p_{\mathbf{A} \mid Q}\left(\mathbf{a}_{c, t} \mid q_{c, t}\right) p_{S \mid \mathbf{A}}\left(s_{c, t} \mid \mathbf{a}_{c, t}\right) p_{B_{c, t} \mid \mathbf{H}_{2, c}, S_{c}^{t-1}, B_{c}^{t-1}}\left(b_{c, t} \mid h_{2, c}, s_{c}^{t-1}, b_{c}^{t-1}\right) \times \\
& \prod_{t=\lceil\gamma L\rceil+1}^{L} p_{Q \mid R}\left(q_{c, t} \mid 1\right) p_{\mathbf{A} \mid Q}\left(a_{c, t} \mid q_{c, t}\right) p_{S \mid \mathbf{A}}\left(s_{c, t} \mid \mathbf{a}_{c, t}\right) p_{B_{c, t} \mid \mathrm{H}_{2, c}, S_{c}^{t-1}, B_{c}^{t-1}}\left(b_{c, t} \mid h_{2, c}, s_{c}^{t-1}, b_{c}^{t-1}\right) \|_{T V} \\
& \leq 3^{(i-2)} \epsilon \text {. }
\end{aligned}
$$

Then, by using the second property of total variation in Lemma 1 for random variables 
$E=\left(\mathbf{A}_{c}^{L}, B_{c}^{L}\right)$ and $F=\left(Q_{c}^{L}, \mathrm{H}_{2, c}, S_{c}^{L}\right)$ and considering that $i-2 \leq N$ we have

$$
\begin{aligned}
\| p_{\mathbf{A}_{c}^{L}, B_{c}^{L}}\left(\mathbf{a}_{c}^{L}, b_{c}^{L}\right)- & \prod_{t=1}^{\lceil\gamma L\rceil} p_{\mathbf{A} \mid R}\left(\mathbf{a}_{c, t} \mid 0\right) p_{B_{c, t} \mid \mathbf{A}_{c}^{t-1}, B_{c}^{t-1}}\left(b_{c, t} \mid \mathbf{a}_{c}^{t-1}, b_{c}^{t-1}\right) \times \\
& \prod_{t=\lceil\gamma L\rceil+1}^{L} p_{\mathbf{A} \mid R}\left(\mathbf{a}_{c, t} \mid 1\right) p_{B_{c, t} \mid \mathbf{A}_{c}^{t-1}, B_{c}^{t-1}}\left(b_{c, t} \mid \mathbf{a}_{c}^{t-1}, b_{c}^{t-1}\right) \|_{T V} \leq 3^{N} \epsilon .
\end{aligned}
$$

Note that by repeating the above arguments, 20 concludes that the inequality (24) holds for the second block as well, i.e., substituting the subscripts ' $c$ ' with ' $s$, the inequality (24) still holds. Let $\mathrm{M}$ be the maximum absolute value of the payoff table. By relating the total variation distance to the payoff, Equation (24) implies that the payoff of team $A$ at the second and the current block (in the $i$-th block for any $i \geq 2$ ) is at most in $2 \mathrm{M} 3^{N} \epsilon$ distance of the payoff of team $A$ when they played i.i.d. according to $p_{\mathbf{A} \mid R}(\mathbf{a} \mid 0)$ in $\gamma$ portion of stages and according to $p_{\mathbf{A} \mid R}(\mathbf{a} \mid 1)$ in the remaining $1-\gamma$ portion of stages. Thus, it suffices to take $N$ large enough so that the effect of the first block in the average payoff of total stages is diminished, and then take $\epsilon$ small enough (consequently take $L$ large enough such that Equations (17) and (22) are satisfied) to make $2 \mathrm{M} 3^{N} \epsilon$ as small as desired.

Remark 18. We have shown that fixing some $k$ and $N$, for sufficiently large $L$, we can achieve the desired payoff in $T=L(k+N)$ stages. If $T$ is not divisible by $k+N$, we can make the first block slightly longer but because $k+N \ll T$ for sufficiently large $T$, this has negligible effect on the achieved payoff.

Remark 19. When player $B$ monitors the actions of the team players imperfectly, we showed that the team players can secure $\pi(\mathbf{A} \mid R)$ for $p_{R Q \mathbf{A} S}$ satisfying (12), (13) and the strict form of (14). For the boundary case when (14) holds with equality, namely when $H(Q \mathbf{A} \mid R S)=H(Q \mid R)$, the proof given above needs a slight modification: let $\epsilon^{\prime}>0$ be an arbitrarily small real number. We add to each block (other than the first block) another subblock of length $\left\lceil\epsilon^{\prime} L\right\rceil$ which we call "the shared randomness banking subblock". In this subblock, the team players play i.i.d. according to $p_{\mathbf{A}}^{(0)}$, as they did in the first block. The 
remaining stages of the block is divided into two subblocks of lengths $\left\lceil\left(1-\epsilon^{\prime}\right) \gamma L\right\rceil$ and $L-\left\lceil\epsilon^{\prime} L\right\rceil-\left\lceil\left(1-\epsilon^{\prime}\right) \gamma L\right\rceil$, where the team players play i.i.d. according to $p_{\mathbf{A} \mid R}(\mathbf{a} \mid 0)$ and $p_{\mathbf{A} \mid R}(\mathbf{a} \mid 1)$, respectively. To do so, the team players need $\left(1-\epsilon^{\prime}\right) H(Q \mid R)$ per stage shared randomness, while they can distill $\epsilon^{\prime} H\left(\mathbf{A}^{(0)} \mid S^{(0)}\right)+\left(1-\epsilon^{\prime}\right) H(\mathbf{A} Q \mid S R)$ per stage shared randomness. Note that since $H(Q \mathbf{A} \mid R S)=H(Q \mid R)$ and $H\left(\mathbf{A}^{(0)} \mid S^{(0)}\right)>0$, the distilled randomness is strictly more than the consumed randomness. Therefore, using a similar argument as we utilized for the strict case of $H(Q \mathbf{A} \mid R S)>H(Q \mid R)$, we can prove that the team players can secure payoffs arbitrarily close to $-\epsilon^{\prime} \mathbf{M}+\left(1-\epsilon^{\prime}\right) \pi(\mathbf{A} \mid R)$. Since $\epsilon^{\prime}$ is arbitrary, we conclude that the team players can secure $\pi(\mathbf{A} \mid R)$.

\subsection{Proof of Theorem 15: Player B can defend $w$}

Since we have given the solution expression in a different form than the one given by Gossner and Tomala (2007), we adapt the proof of Gossner and Tomala (2007) to our solution form.

In this subsection we assume that at the end of each stage $t$, the players of team $A$ in addition to $\mathbf{A}_{t}$ and $B_{t}$, observe the signal $S_{t}$. We show that even with the more information available to players of team $A$, player $B$ can still defend $w$. Let $\sigma$ be an arbitrary strategy for team $A$. We generate strategy $\tau$ for player $B$ as follows: given $h_{2}^{t}$, an arbitrary history of observations of player $B$ until stage $t, \tau_{t}\left(h_{2}^{t}\right)$ is the best choice of player $B$ that minimizes the expected payoff at stage $t$, i.e.,

$$
\tau_{t}\left(h_{2}^{t}\right) \in \underset{b \in \mathcal{B}}{\arg \min } \mathbb{E}_{\sigma}\left[u_{\mathbf{A}_{t}, b} \mid \mathrm{H}_{2}^{t}=h_{2}^{t}\right]
$$

where $\mathbb{E}_{\sigma}$ denotes the expectation with respect to the probability distribution induced by $\sigma$. Let team $A$ and player $B$ play with respective strategies $\sigma$ and $\tau$ and let $\mathbf{A}^{T}, B^{T}$ and $S^{T}$ denote the sequence of actions and signals generated during the $T$ stages of the game. Define:

$$
R=\left(I, B^{I-1}, S^{I-1}\right), \quad Q=\left(I, \mathbf{A}^{I-1}, B^{I-1}, S^{I-1}\right), \quad \tilde{\mathbf{A}}=\mathbf{A}_{I} \quad \text { and } \quad \tilde{S}=S_{I},
$$


where $I$ is a uniformly distributed random variable on $\mathcal{I}=\{1,2, \ldots, T\}$ and independent of $\left(\mathbf{A}^{T}, B^{T}, S^{T}\right)$. Random variable $I$ is the so-called time sharing random variable. Note that $R$ is a function of $Q$. Therefore,

$$
p_{\tilde{S}, \tilde{\mathbf{A}}, R, Q}(s, \mathbf{a}, r, q)=p_{R Q}(r, q) p_{\tilde{\mathbf{A}} \mid Q}(\mathbf{a} \mid q) p_{S \mid \mathbf{A}}(s \mid \mathbf{a}) .
$$

Since the action profile $\mathbf{A}_{t}$ is implemented distributively by conditioning on $\left(\mathbf{A}^{t-1}, B^{t-1}, S^{t-1}\right)$, the conditional distribution $p_{\tilde{\mathbf{A}} \mid Q}(\mathbf{a} \mid q)$ belongs to $\Pi$, i.e., $p_{\tilde{\mathbf{A}} \mid Q}(\mathbf{a} \mid q) \in \Pi$. Next, we need to show that $H(Q \tilde{\mathbf{A}} \mid \tilde{S} R) \geq H(Q \mid R)$. Note that

$$
H(Q \tilde{\mathbf{A}} \mid \tilde{S} R)-H(Q \mid R)=H(\tilde{\mathbf{A}} \tilde{S} \mid Q R)-H(\tilde{S} \mid R)
$$

The inequality $H(\tilde{\mathbf{A}} \tilde{S} \mid Q R)-H(\tilde{S} \mid R) \geq 0$ follows from

$$
\begin{aligned}
H(\tilde{\mathbf{A}} \tilde{S} \mid Q R)- & H(\tilde{S} \mid R)=H\left(\mathbf{A}_{I} S_{I} \mid I, \mathbf{A}^{I-1}, B^{I-1}, S^{I-1}\right)-H\left(S_{I} \mid I, B^{I-1}, S^{I-1}\right) \\
& =\sum_{t=1}^{T} \frac{1}{T}\left(H\left(\mathbf{A}_{t}, S_{t} \mid \mathbf{A}^{t-1}, B^{t-1}, S^{t-1}\right)-H\left(S_{t} \mid B^{t-1}, S^{t-1}\right)\right) \\
& =\sum_{t=1}^{T} \frac{1}{T}\left(H\left(\mathbf{A}^{t} \mid B^{t}, S^{t}\right)-H\left(\mathbf{A}^{t-1} \mid B^{t-1}, S^{t-1}\right)\right) \\
& =\frac{1}{T} H\left(\mathbf{A}^{T} \mid B^{T}, S^{T}\right) \geq 0
\end{aligned}
$$

where (26) follows from the fact that $I$ is uniform and independent of $\left(\mathbf{A}^{T}, B^{T}, S^{T}\right)$, and 27) follows from

$$
\begin{aligned}
& H\left(\mathbf{A}^{t} \mid B^{t}, S^{t}\right)-H\left(\mathbf{A}^{t-1} \mid B^{t-1}, S^{t-1}\right) \\
& =H\left(\mathbf{A}^{t-1} \mid B^{t}, S^{t}\right)+H\left(\mathbf{A}_{t} \mid B^{t}, S^{t}, \mathbf{A}^{t-1}\right)-H\left(\mathbf{A}^{t-1} \mid B^{t-1}, S^{t-1}\right) \\
& =H\left(\mathbf{A}^{t-1}, B_{t}, S_{t} \mid B^{t-1}, S^{t-1}\right)-H\left(B_{t}, S_{t} \mid B^{t-1}, S^{t-1}\right)+H\left(\mathbf{A}_{t}, B_{t}, S_{t} \mid \mathbf{A}^{t-1}, B^{t-1}, S^{t-1}\right) \\
& \quad-H\left(B_{t}, S_{t} \mid \mathbf{A}^{t-1}, B^{t-1}, S^{t-1}\right)-H\left(\mathbf{A}^{t-1} \mid B^{t-1}, S^{t-1}\right) \\
& =H\left(\mathbf{A}_{t}, B_{t}, S_{t} \mid \mathbf{A}^{t-1}, B^{t-1}, S^{t-1}\right)-H\left(B_{t}, S_{t} \mid B^{t-1}, S^{t-1}\right) \\
& =H\left(\mathbf{A}_{t}, S_{t} \mid \mathbf{A}^{t-1}, B^{t-1}, S^{t-1}\right)-H\left(S_{t} \mid B^{t-1}, S^{t-1}\right)+H\left(B_{t} \mid \mathbf{A}^{t}, B^{t-1}, S^{t}\right)-H\left(B_{t} \mid B^{t-1}, S^{t}\right) \\
& =H\left(\mathbf{A}_{t}, S_{t} \mid \mathbf{A}^{t-1}, B^{t-1}, S^{t-1}\right)-H\left(S_{t} \mid B^{t-1}, S^{t-1}\right)
\end{aligned}
$$


where (28) holds because the random variable $B_{t}$ is conditionally independent of $\left(\mathbf{A}^{t}, S_{t}\right)$ given $\left(B^{t-1}, S^{t-1}\right)$.

Now we relate the payoff of $\sigma$ and $\tau$ to $R$ and $\tilde{\mathbf{A}}$ :

$$
\begin{aligned}
\lambda_{T}(\sigma, \tau) & =\sum_{t=1}^{T} \frac{1}{T} \sum_{b^{t-1}, s^{t-1}} p_{B^{t-1}, S^{t-1}}\left(b^{t-1}, s^{t-1}\right) \min _{b \in \mathcal{B}} \mathbb{E}\left[u_{\mathbf{A}_{t}, b} \mid B^{t-1}=b^{t-1}, S^{t-1}=s^{t-1}\right] \\
& =\sum_{r} p_{R}(r) \min _{b \in \mathcal{B}} \mathbb{E}\left[u_{\tilde{\mathbf{A}}, b} \mid R=r\right]=\pi(\tilde{\mathbf{A}} \mid R) .
\end{aligned}
$$

We have identified random variables $(R, Q, \tilde{\mathbf{A}}, \tilde{S})$ satisfying the constraints of the problem, except for the cardinality bounds on $R$ and $Q$. Cardinality of $R$ and $Q$ can be reduced using the standard arguments such as the support lemma of (El Gamal and Kim, 2011, Appendix C) or the Fenchel-Bunt extension to the Caratheodory's theorem. We leave the argument on the reduction of the cardinality of $R$ and $Q$ to Proposition 20. According to Proposition 20, given the random variables $(R, Q, \tilde{\mathbf{A}}, \tilde{S})$ satisfying the constraints of the problem except for the cardinality bounds on $R$ and $Q$, we can identify other random variables $\left(R^{\prime}, Q^{\prime}, \mathbf{A}^{\prime}, S^{\prime}\right)$ such that they satisfy all the constraints of the problem.

Proposition 20. Let $\mathbf{A} \in \mathcal{A}, S \in \mathcal{S}, R \in \mathcal{R}$ and $Q \in \mathcal{Q}$ have a joint distribution satisfying Equations (12)-(14) along with

$$
\pi(\mathbf{A} \mid R) \geq \beta
$$

where $\mathcal{R}$ and $\mathcal{Q}$ are finite sets with arbitrary cardinalities and $\beta$ is an arbitrary real number. There exist random variables $\mathbf{A}^{\prime} \in \mathcal{A}, S^{\prime} \in \mathcal{S}, R^{\prime} \in \mathcal{R}^{\prime}$ and $Q^{\prime} \in \mathcal{Q}^{\prime}$ such that $\left|\mathcal{R}^{\prime}\right|=2$, $\left|\mathcal{Q}^{\prime}\right|=2|\mathcal{A}|$ and the joint distribution on $\left(\mathbf{A}^{\prime}, S^{\prime}, R^{\prime}, Q^{\prime}\right)$ satisfies Equations (12)-(14) and (30).

The proof of Proposition 20 is provided in Appendix B.

\section{Computation of min-entropy function}

Consider a one-shot zero-sum game between players Alice (maximizer) and Bob (minimizer) and let $\mathcal{J}(\mathrm{h})$ be the maximum expected payoff that Alice can secure (regardless 
of what Bob plays) by playing mixed actions of entropy at most $\mathrm{h}$ (as defined in Equation (2)). As stated in Theorems 3 and $9, \mathcal{J}(\mathrm{h})$ characterizes the maxmin values of the repeated game with bounded entropy (Section 3.1) and the repeated game with leaked randomness (Section 3.3). The main goal of this section is to study the computational aspects of $\mathcal{J}(\mathrm{h})$.

Let $F(w)$ denote the inverse function of $\mathcal{J}(\mathrm{h}) . F(w)$ is the minimum entropy that Alice needs to secure payoff $w$; thus, we call it the min-entropy function. To compute $F(w)$, one has to minimize the concave function of entropy over the polytope of mixed actions that secure payoff $w$ for Alice. The problem of entropy minimization over a polytope is a standard problem and is known to be NP-hard (see Kovačević et al. (2012)). Thus, the computation of $F(w)$ (or $\mathcal{J}(\mathrm{h}))$ is NP-hard. In this section, we study $\mathcal{J}(\mathrm{h})$ thorough its inverse, $F(w)$, and provide some computationally efficient upper and lower bounds for it.

\subsection{Problem statement}

Consider a zero-sum game between players Alice $(A)$ and Bob $(B)$ with respective pure strategies sets $\mathcal{A}=\{1, \ldots, n\}$ and $\mathcal{B}=\left\{1, \ldots, n^{\prime}\right\}$, where $n$ and $n^{\prime}$ are natural numbers. The payoff matrix is denoted by $\mathrm{U}=\left[u_{i, j}\right]$, where $u_{i, j}$ is the real valued payoff that player $A$ gets from player $B$ when $i \in \mathcal{A}$ and $j \in \mathcal{B}$ are played. Player $A$ (player $B$ ) wishes to maximize (minimize) the expected payoff. The set of all randomized strategies of players $A$ and $B$ are denoted by $\Delta(\mathcal{A})$ and $\Delta(\mathcal{B})$ respectively, which are the probability simplexes on sample spaces $\mathcal{A}$ and $\mathcal{B}$ respectively. Thus, Alice's strategy corresponds to a pmf $\mathbf{p}=\left(p_{1}, p_{2}, \ldots, p_{n}\right)$, which can be also illustrated as a column vector with non-negative entries that add up to one.

Assume that player $A$ uses randomized strategy p. Then, the payoff of Alice if Bob plays $j \in \mathcal{B}$ is $\sum_{i} p_{i} u_{i, j}$. We say that Alice secures payoff $w$ with randomized strategy $\mathbf{p}$ (regardless of the action of player $B$ ) if $\sum_{i} p_{i} u_{i, j} \geq w$ for all $j \in \mathcal{B}$. Thus, the set of all distributions that guarantee payoff $w$ for player $A$ can be expressed as

$$
\mathcal{P}_{\mathrm{U}}(w)=\left\{\mathbf{p} \in \Delta(\mathcal{A}): \mathbf{p}^{T} \mathrm{U} \geq w \mathbf{1}^{T}\right\}
$$


where $\mathbf{p}^{T}$ is the transpose of the column vector $\mathbf{p}, \mathbf{1}$ is a column vector of all ones and $\mathbf{v}_{1} \geq \mathbf{v}_{2}$ means any element of $\mathbf{v}_{1}$ is greater than or equal to the corresponding element at $\mathbf{v}_{2}$.

We define

$$
F_{\mathrm{U}}(w) \triangleq \min _{\mathbf{p} \in \mathcal{P}_{\mathrm{U}}(w)} H(\mathbf{p}) .
$$

If the set $\mathcal{P}_{\mathrm{U}}(w)$ is empty, we set $F_{\mathrm{U}}(w)=+\infty$.

A remark on notation: Two-player zero-sum games are completely characterized by their payoff matrix. Hence, for the sake of simplicity, we will call two-player zero-sum games with their payoff matrix. Thus, game U refers to a game with payoff table $\mathrm{U}$.

Definition 21. Given a game $\mathrm{U}=\left[u_{i, j}\right]$, parameters $\underline{m}, \bar{m}, v$ and $w^{*}$ are defined as:

- $\underline{m}(\bar{m})$ is the minimum (maximum) element of matrix $\mathrm{U}: \underline{m}=\min _{i, j} u_{i, j}(\bar{m}=$ $\left.\max _{i, j} u_{i, j}\right)$.

- $v$ is the maximum payoff secured by pure actions (pure-strategy security level): $v=\max _{i} \min _{j} u_{i, j}$.

- $w^{*}$ is the value of the game $\mathrm{U}$, which is the maximum guaranteed payoff with unlimited access to random sources:

$$
w^{*}=\max _{w: \mathcal{P}_{\mathrm{u}}(w) \neq \emptyset} w
$$

Note that by definition, $\underline{m} \leq v \leq w^{*} \leq \bar{m}$.

According to Definition 21, $v$ is the payoff that is guaranteed without consumption of any randomness, whereas $w^{*}$ is the maximum guaranteed payoff when unlimited randomness is available. Thus, it is interesting to consider the min-entropy function $F_{\mathrm{U}}(w)$ in the domain $v \leq w \leq w^{*}$. If $w \leq v$, then $F_{\mathrm{U}}(w)=0$; if $w>w^{*}$ the feasible set of optimization problem in 32 is empty and $F_{\mathrm{U}}(w)=+\infty$. When $v \leq w \leq w^{*}$, the function $F_{\mathrm{U}}(w)$ is not necessarily convex or concave as a function of $w$ : it is strictly increasing and piecewise concave (Neyman and Okada, 2000, p. 241). ${ }^{2}$

\footnotetext{
${ }^{2}$ This property is stated in Neyman and Okada 2000 in terms of the function $J(\cdot)$.
} 


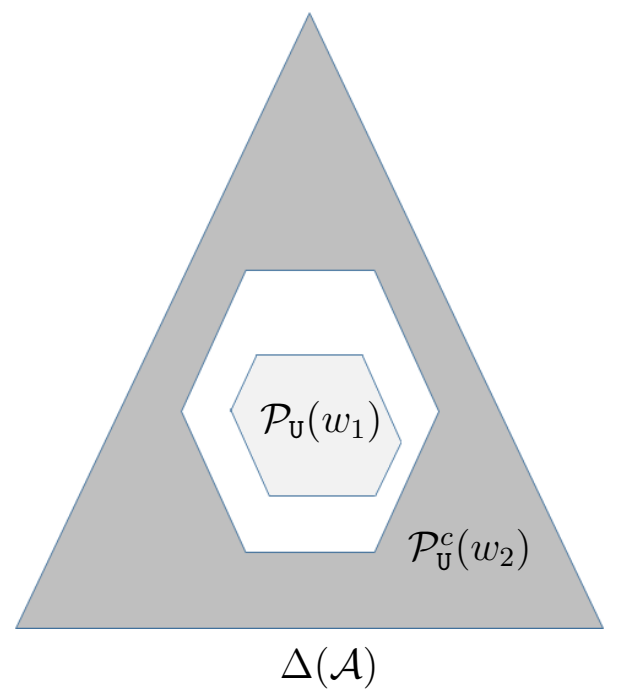

Figure 2: Illustration of $\mathcal{P}_{\mathrm{U}}\left(w_{1}\right), \mathcal{P}_{\mathrm{U}}^{c}\left(w_{2}\right)$ and $\Delta(\mathcal{A})$

\subsection{On the set $\mathcal{P}_{\mathrm{U}}(w)$}

The function $F_{\mathrm{U}}(w)$ is defined in (32) using $\mathcal{P}_{\mathrm{U}}(w)$, the set of all distributions that guarantee a security level $w$ for player $A$. Observe that

$$
\mathcal{P}_{\mathrm{U}}(w)=\left\{\mathbf{p} \in \Delta(\mathcal{A}): \mathbf{p}^{T} \mathrm{U} \geq w \mathbf{1}^{T}\right\}
$$

is a polytope defined via some linear constraints. As the matrix $\mathrm{U}$ is completely arbitrary, with a change of variables, one can convert it to many different equivalent polytopes.

We only need to study $\mathcal{P}_{\mathrm{U}}(w)$ for $v \leq w \leq w^{*}$. It is immediate from the definition of $\mathcal{P}_{\mathrm{U}}(w)$ that this set is decreasing in $w$, i.e., for any $w_{1} \geq w_{2}$,

$$
\mathcal{P}_{\mathrm{U}}\left(w_{1}\right) \subseteq \mathcal{P}_{\mathrm{U}}\left(w_{2}\right)
$$

We are interested to see if the inclusion in (35) is strict, and if yes, quantify to what extent it is. To do this, we look at the distance between the set $\mathcal{P}_{\mathrm{U}}\left(w_{1}\right)$ and the compliment of $\mathcal{P}_{\mathrm{U}}\left(w_{2}\right)$ (that is $\left.\mathcal{P}_{\mathrm{U}}^{c}\left(w_{2}\right)=\Delta(\mathcal{A})-\mathcal{P}_{\mathrm{U}}\left(w_{2}\right)\right)$. The sets $\mathcal{P}_{\mathrm{U}}\left(w_{1}\right), \mathcal{P}_{\mathrm{U}}^{c}\left(w_{2}\right)$ and $\Delta(\mathcal{A})$ are illustrated in Figure 2. The distance between any two sets can be defined as

$$
d\left(\mathcal{S}_{1}, \mathcal{S}_{2}\right) \triangleq \inf _{\mathbf{p} \in \mathcal{S}_{1}, \mathbf{q} \in \mathcal{S}_{2}} d(\mathbf{p}, \mathbf{q})
$$

where $d(\mathbf{p}, \mathbf{q})$ can be any arbitrary distance measure. The standard option is the total 
variation distance

$$
d_{1}(\mathbf{p}, \mathbf{q})=\frac{1}{2} \sum_{i}\left|p_{i}-q_{i}\right|
$$

With this choice of the distance, we have

Theorem 22. For any $w_{1}$ and $w_{2}$ satisfying $v \leq w_{2} \leq w_{1} \leq w^{*}$, we have

$$
d_{1}\left(\mathcal{P}_{\mathrm{U}}^{c}\left(w_{2}\right), \mathcal{P}_{\mathrm{U}}\left(w_{1}\right)\right) \geq \frac{\left|w_{1}-w_{2}\right|}{|\bar{m}-\underline{m}|} .
$$

Observe that the quantities $\underline{m}$ and $\bar{m}$ can be simply computed from $\mathrm{U}$. The idea of the proof of Theorem 22 is standard (e.g., see (Gossner and Vieille, 2002, eq. (4)) for a similar derivation), but is included in Section 6.3 for completeness.

In this paper, we propose the use of the Rényi divergence of order two between $\mathbf{p}$ and $\mathbf{q}$ to quantify the distance between two distributions: ${ }^{3}$

$$
d_{2}(\mathbf{p}, \mathbf{q}) \triangleq \log \left(\sum_{i} \frac{p_{i}^{2}}{q_{i}}\right)=\log \left(1+\chi^{2}(\mathbf{p}, \mathbf{q})\right) .
$$

Our first result gives the following bound:

Theorem 23. For any $w_{1}, w_{2}$ satisfying $v \leq w_{2} \leq w_{1} \leq w^{*}$, we have

$$
d_{2}\left(\mathcal{P}_{\mathrm{U}}^{c}\left(w_{2}\right), \mathcal{P}_{\mathrm{U}}\left(w_{1}\right)\right) \geq \log \left(1+\frac{\left(w_{1}-w_{2}\right)^{2}}{\left(w_{1}-\underline{m}\right)\left(\bar{m}-w_{1}\right)}\right) .
$$

The proof can be found in Section 6.3. The above result is derived by a probabilistic approach, which we believe is novel in the context of linear programming.

Our second result is less crucial, but still useful. It gives a compact formula for the supporting hyperplanes of $\mathcal{P}_{\mathrm{U}}(w)$ in terms of the Nash equilibrium of a game. We need a definition:

Definition 24. Val(U) denotes the value of a two-player zero-sum game with payoff table $\mathrm{U}=\left[u_{i, j}\right]$.

\footnotetext{
${ }^{3}$ In this definition, we set $p_{i}^{2} / q_{i}$ to be zero if $p_{i}=q_{i}=0$, and infinity if $p_{i}>0$ while $q_{i}=0$. We have that $d_{2}(\mathbf{p}, \mathbf{q}) \geq 0$, and $d_{2}(\mathbf{p}, \mathbf{q})=0$ if and only if $\mathbf{p}=\mathbf{q}$.
} 
$\mathrm{Val}(\mathrm{U})$ is the maximum value that Alice can guarantee using arbitrary mixed strategies. It is known that in a zero-sum game, while the game might have multiple Nash equilibria, the value of Alice in all of the equilibria is the same (See e.g., Narahari (2014), p.145).

Given values $a_{1}, a_{2}, \ldots, a_{n} \in \mathbb{R}$, let us construct a new table whose $(i, j)$ entry is $\tilde{u}_{i j}=u_{i, j}+a_{i}$. In other words, we add $a_{i}$ to the entries in the $i$-th row of $\mathrm{U}$. The new table can be expressed as $\tilde{\mathrm{U}}=\mathrm{U}+\mathbf{a} \cdot \mathbf{1}^{T}$, where $\mathbf{a}$ is a column vector whose entries are $a_{1}, a_{2}, \ldots, a_{n}$ and $\mathbf{1}^{T}$ is a row vector of all ones. Observe that the table $\tilde{\mathrm{U}}$ can be intuitively understood as giving an additional incentive $a_{i}$ to Alice for playing her $i$-th action (it is actually a disincentive or "tax" if $a_{i}<0$ ).

Theorem 25. The set $\mathcal{P}_{\mathrm{U}}(w)$ can be characterized as follows:

$$
\mathcal{P}_{\mathrm{U}}(w)=\left\{\mathbf{p} \in \Delta(\mathcal{A}) \mid \sum_{i} a_{i} p_{i} \leq \operatorname{Val}\left(\mathrm{U}+\mathbf{a} \mathbf{1}^{T}\right)-w, \quad \forall \mathbf{a}\right\} .
$$

Remark 26. Note that $\max _{\mathbf{p} \in \mathcal{P}_{\mathrm{U}}(w)}\left(\sum_{i} a_{i} p_{i}\right)$ is simply a linear program. The Equivalence of linear program and Nash equilibria is known in the literature (Dantzig (1951); Adler (2013)). However, our construction of the game $\tilde{\mathrm{U}}$ based on incentive or tax is new to best of our knowledge.

In Section 6.4, we give the proof of Theorem 25 as well as a geometric picture of the Nash equilibrium strategies of Alice.

\subsubsection{On the min-entropy function}

We begin with a property of the min-entropy function. To state the property, we need the following definition:

Definition 27. Consider two games with payoff matrixes $\mathrm{U}_{1}$ and $\mathrm{U}_{2}$. Let $\mathrm{U}_{3}=\mathrm{U}_{1} \oplus \mathrm{U}_{2}$ be the direct-sum of $\mathrm{U}_{1}$ and $\mathrm{U}_{2} . \mathrm{U}_{3}$ defines a new game in which players simultaneously play one instance of $\mathrm{U}_{1}$ and one instance of $\mathrm{U}_{2}$ and the resulting payoff is the sum of payoffs from $\mathrm{U}_{1}$ and $\mathrm{U}_{2}$.

Theorem 28. For every game $\mathrm{U}, F_{\mathrm{U} \oplus \mathrm{U}}(w)=F_{\mathrm{U}}(w / 2)$. Similarly, for every natural number $k, F_{\oplus^{k} \mathrm{U}}(w)=F_{\mathrm{U}}(w / k)$, where $\oplus^{k} \mathrm{U}$ is $k$ times direct sum of $\mathrm{U}$. 
Proof of Theorem 28 is provided in Section 6.5 .

An application of the above theorem is that given an expression $G_{\mathrm{U}}(w)$ that bounds $F_{\mathrm{U}}(w)$ from below for all $w$ and $\mathrm{U}$, we can conclude that $G_{\oplus^{k} \mathrm{U}}(k w) \leq F_{\oplus^{k} \mathrm{U}}(k w)=F_{\mathrm{U}}(w)$. Thus, $\max _{k} G_{\oplus^{k} \mathrm{U}}(k w)$ is also a (potentially better) lower bound to $F_{\mathrm{U}}(w)$. As a result, we expect that a "good" lower (or upper bound) on $F_{\mathrm{U}}(w)$ should have the correct scaling behavior as we simultaneously play more and more copies of the game.

\subsection{Lower and upper bounds on the min-entropy function}

The min-entropy function $F_{\mathrm{U}}(w)$ in $(32)$ is the minimum of a concave function on a polytope $\mathcal{P}_{\mathrm{U}}(w)$. This minimum occurs at a vertex of $\mathcal{P}_{\mathrm{U}}(w)$. This leads to a search in the exponentially large set of vertexes of the polytope $\mathcal{P}_{\mathrm{U}}(w)$, which is computationally hard. We desire to find bounds on $F_{\mathrm{U}}(w)$ that are either explicit, or else can be computed in polynomial time. Observe that

$$
F_{\mathrm{U}}(w)=\min _{\mathbf{p} \in \mathcal{P}_{\mathrm{U}}(w)} H(\mathbf{p})=\log (|\mathcal{A}|)-\max _{\mathbf{p} \in \mathcal{P}_{\mathrm{U}}(w)} D\left(\mathbf{p} \| \mathbf{p}^{\mathbf{u}}\right),
$$

where $\mathbf{p}^{\mathbf{u}}$ is the uniform distribution over $\mathcal{A}$, and $D(\mathbf{p} \| \mathbf{q})=\sum_{i} p_{i} \log \left(p_{i} / q_{i}\right)$ is the Kullback-Leibler (KL) divergence. Thus, we are interested in finding the vertex of $\mathcal{P}_{\mathrm{U}}(w)$ which has maximum distance from the uniform distribution (with respect to KL divergence).

Lower bound: To prove lower bounds for

$$
F_{\mathrm{U}}(w)=\min _{\mathbf{p} \in \mathcal{P}_{\mathrm{U}}(w)} H(\mathbf{p}),
$$

one idea is to replace the entropy function with a smaller function and compute the minimum over $\mathcal{P}_{\mathrm{U}}(w)$. The second idea is to minimize the min-entropy function $F_{\mathrm{U}}(w)$ over all payoff tables with given properties such as $\underline{m}, \bar{m}$ and $v$. Another idea is to relax the set of distributions $\mathcal{P}_{\mathrm{U}}(w)$ and replace it with a potentially bigger set. We proceed with the first idea, then elaborate on the second idea and finally comment on the third idea. 
Using the fact that the Rényi entropy is decreasing in its order, we obtain that for any $\alpha>1$

$$
F_{\mathrm{U}}(w)=\min _{\mathbf{p} \in \mathcal{P}_{\mathrm{U}}(w)} H(\mathbf{p}) \geq \min _{\mathbf{p} \in \mathcal{P}_{\mathrm{U}}(w)} H_{\alpha}(\mathbf{p}),
$$

where $H_{\alpha}(\mathbf{p})$ is the Rényi entropy of order $\alpha$ :

$$
H_{\alpha}(\mathbf{p})=\frac{1}{1-\alpha} \log _{2}\left(\sum_{i} p_{i}^{\alpha}\right) .
$$

The case of $\alpha=2$ is related to the Euclidean norm and results in an optimization problem similar to the one given in (36) for the Euclidean norm instead of the KL divergence, which is still not tractable. However, the case $\alpha=\infty$ relates to the maximum norm and results in the following lower bound:

$$
G_{\mathrm{U}}^{(1)}(w)=-\log _{2}\left(\max _{i \in \mathcal{A}} \max _{\mathbf{p} \in \mathcal{P}_{\mathrm{U}}(w)} p_{i}\right) .
$$

For each $i$, the problem of finding the maximum of $p_{i}$ over $\mathbf{p} \in \mathcal{P}_{\mathrm{U}}(w)$ is a linear program. From Theorem 25, we can find an upper bound on the value of this linear program, yielding

$$
G_{\mathrm{U}}^{(1)}(w) \geq-\log _{2} \max _{i}\left(\operatorname{Val}\left(\mathrm{U}+\mathbf{e}_{i} \mathbf{1}^{T}\right)-w\right)
$$

where $\mathbf{e}_{i}$ is a vector of length $|\mathcal{A}|=n$ whose $i$-th coordinate is one, and all its other coordinates are zero. The lower bound $G_{\mathrm{U}}^{(1)}(w)$ or its relaxed version in 39 can be found in polynomial time, even though they are not in explicit forms.

To obtain an explicit lower bound, observe that $\log _{2}\left(1 / p_{i}\right)=d_{2}\left(\mathbf{e}_{i}, \mathbf{p}\right)$. Note that the vector $\mathbf{e}_{i}$ is a probability vector associated to a deterministic random variable that chooses $i$ with probability one. Take some $\epsilon>0$. By definition, deterministic strategies cannot 
secure a payoff of more than $v$. Hence, $\mathbf{e}_{i} \in \mathcal{P}_{\mathrm{U}}^{c}(v+\epsilon)$, so we have

$$
\begin{aligned}
G_{\mathrm{U}}^{(1)}(w) & =-\log _{2}\left(\max _{\mathbf{p} \in \mathcal{P}_{\mathrm{U}}(w)} \max _{i \in \mathcal{A}} p_{i}\right) \\
& =\min _{\mathbf{p} \in \mathcal{P}_{\mathrm{U}}(w)} \min _{i \in \mathcal{A}} \log _{2}\left(\frac{1}{p_{i}}\right) \\
& =\min _{\mathbf{p} \in \mathcal{P}_{\mathrm{U}}(w)} \min _{i \in \mathcal{A}} d_{2}\left(\mathbf{e}_{i}, \mathbf{p}\right) \\
& \geq \min _{\mathbf{p} \in \mathcal{P}_{\mathrm{U}}(w)} \min _{\mathbf{q} \in P_{\mathrm{U}}^{c}(v+\epsilon)} d_{2}(\mathbf{q}, \mathbf{p}) \\
& \geq \log _{2}\left(1+\frac{(w-v-\epsilon)^{2}}{(w-\underline{m})(\bar{m}-w)}\right),
\end{aligned}
$$

where (40) follows from Theorem 23. Letting $\epsilon \rightarrow 0$, we obtain

$$
F_{\mathrm{U}}(w) \geq G_{\mathrm{U}}^{(2)}(w) \triangleq \log _{2}\left(1+\frac{(w-v)^{2}}{(w-\underline{m})(\bar{m}-w)}\right) \quad \forall w: v \leq w \leq w^{*} .
$$

With a similar argument and using Theorem 22 along with the fact that $p_{i}=1-$ $d_{1}\left(\mathbf{e}_{i}, \mathbf{p}\right)$, we obtain the following lower bound:

$$
F_{\mathrm{U}}(w) \geq G_{\mathrm{U}}^{(3)}(w) \triangleq-\log _{2}\left(1-\frac{w-v}{\bar{m}-\underline{m}}\right) \quad \forall w: v \leq w \leq w^{*} .
$$

Observe that when $v=\underline{m}, G_{\mathrm{U}}^{(2)}(w)$ equals $G_{\mathrm{U}}^{(3)}(w)$. When $v \neq \underline{m}$, a simple calculation shows that $G_{\mathrm{U}}^{(2)}(w) \geq G_{\mathrm{U}}^{(3)}(w)$ if and only if $w \geq(\bar{m}+v) / 2$.

Example 29. Consider a game with payoff matrix:

$$
\mathrm{U}=\left[\begin{array}{ccc}
-1 & 1 & 1 \\
1 & 0.5 & 1 \\
1 & 1 & 0.5
\end{array}\right]
$$

From the payoff matrix we have $v=.5, \underline{m}=-1, \bar{m}=1$ and $w^{*}=0.778$. Therefore, for $w \geq 0.75, G_{\mathrm{U}}^{(2)}(w)$ gives a better lower bound than $G_{\mathrm{U}}^{(3)}(w)$ on $F_{\mathrm{U}}(w)$.

Remark 30. One can inspect that just like the min-entropy function, the explicit lower bounds $G_{\mathrm{U}}^{(2)}(w)$ and $G_{\mathrm{U}}^{(3)}(w)$ satisfy

$$
G_{\mathrm{U} \oplus \mathrm{U}}^{(i)}(w)=G_{\mathrm{U}}^{(i)}(w / 2), \quad i=2,3
$$


thus, have the correct scaling behavior. Additionally, by replacing entropy with Rényi entropy in the proof of Theorem 28, one also obtains that

$$
G_{\mathrm{U} \oplus \mathrm{U}}^{(1)}(w)=G_{\mathrm{U}}^{(1)}(w / 2)
$$

The function $G_{\mathrm{U}}^{(2)}(w)$ has second derivative for all $v \leq w \leq w^{*}$, while the second derivative of the piecewise concave function $F_{\mathrm{U}}(w)$ is defined everywhere except for a finite number of kink points. The second derivative of the function $G_{\mathrm{U}}^{(2)}(w)$ may be positive or negative, while $F_{\mathrm{U}}(w)$ is piecewise concave. On the other hand, the function $G_{\mathrm{U}}^{(1)}(w)$ is piecewise convex. The reason is that if $\max _{i \in \mathcal{A}} \max _{\mathbf{p} \in \mathcal{P}_{\mathrm{U}}(w)} p_{i}$ is attained by $i^{*}$ and a particular vertex of $\mathcal{P}_{\mathrm{U}}(w)$ for $w \in\left[w_{1}, w_{2}\right]$, in this interval $\max _{i \in \mathcal{A}} \max _{\mathbf{p} \in \mathcal{P}_{\mathrm{U}}(w)} p_{i}$ varies linearly in $w$. Then, convexity of $-\log _{2}(\cdot)$ results in convexity of $G_{\mathrm{U}}^{(1)}(w)$ in the interval $\left[w_{1}, w_{2}\right]$.

Remark 31. Inequality (40) shows that

$$
\max _{\mathbf{p} \in \mathcal{P}_{\mathbf{U}}(w)} p_{i} \leq\left(1+\frac{(w-v)^{2}}{(w-\underline{m})(\bar{m}-w)}\right)^{-1} \quad \forall w: v \leq w \leq w^{*} \text { and } \forall i=1, \ldots, n,
$$

which gives an upper bound for the linear programming of $\max _{\mathbf{p} \in \mathcal{P}_{\mathrm{U}}(w)} p_{i}$. Note that $\mathcal{P}_{\mathrm{U}}(w)$ is a very generic polytope, parameterized by a variable $w$. By a change of variables (scaling and shifting), one can convert $\max _{\mathbf{p} \in \mathcal{P}_{\mathrm{U}}(w)} p_{i}$ to a wide class of linear programs (with no immediate connection to the probability simplex), and then use the bound given in (41).

Let $\underline{m}, \bar{m}$ and $v$ be the minimum entry, maximum entry and pure strategy security level of the payoff table U. The lower bounds $G_{\mathrm{U}}^{(2)}(w)$ and $G_{\mathrm{U}}^{(3)}(w)$, just rely on the parameters $\underline{m}, \bar{m}$ and $v$. We seek to answer the following question: given that we just know the parameters $\underline{m}, \bar{m}$ and $v$ from payoff table $\mathrm{U}$, what is the tightest lower bound for the min-entropy function? To compute the tightest lower bound it suffices to minimize the min-entropy function over all payoff tables (of arbitrary size) with parameters $\underline{m}, \bar{m}$ and $v$. Let denote this lower bound by $G_{\mathrm{U}}^{(4)}(w)$, then,

$$
G_{\mathrm{U}}^{(4)}(w)=\min _{\mathrm{U}^{\prime}} \min _{\mathbf{p} \in \mathcal{P}_{\mathrm{U}^{\prime}}(w)} H(\mathbf{p})
$$


where the first minimization is computed over all payoff tables $\mathrm{U}^{\prime}$ (of arbitrary size) with minimum entry $\underline{m}$, maximum entry $\bar{m}$ and pure-strategy security level $v$. We simplify the expression of Equation (42) in the following theorem:

Theorem 32. Let $\underline{m}, \bar{m}, v$ and $w$ be some known real numbers such that $\underline{m} \leq v \leq w \leq \bar{m}$. We have

$$
\begin{aligned}
G_{\mathrm{U}}^{(4)}(w)=\min _{\mathrm{U}^{\prime}} \min _{\mathbf{p} \in \mathcal{P}_{\mathrm{U}^{\prime}}(w)} H(\mathbf{p})=-\left\lfloor\frac{\bar{m}-v}{\bar{m}-w}\right\rfloor \frac{\bar{m}-w}{\bar{m}-v} \log \left(\frac{\bar{m}-w}{\bar{m}-v}\right)- & \\
& \left(1-\left\lfloor\frac{\bar{m}-v}{\bar{m}-w}\right\rfloor \frac{\bar{m}-w}{\bar{m}-v}\right) \log \left(1-\left\lfloor\frac{\bar{m}-v}{\bar{m}-w}\right\rfloor \frac{\bar{m}-w}{\bar{m}-v}\right),
\end{aligned}
$$

where the first minimization is computed over all payoff tables $\mathrm{U}^{\prime}$ (of arbitrary size) with minimum entry $\underline{m}$, maximum entry $\bar{m}$ and pure-strategy security level $v$, and $\lfloor a\rfloor$ is the greatest integer smaller than or equal to a.

The proof of the above theorem is provided in Section 6.6. Theorem 32 gives another explicit form lower bound for the min-entropy function which is optimal in the sense that it is the tightest lower bound that utilizes just the information of minimum entry, maximum entry and pure-strategy security level of the payoff table.

Remark 33. The bound $G_{\mathrm{U}}^{(4)}(w)$ does not depend on the minimum entry $\underline{m}$. This is because in the minimization problem of Equation (42), we do not restrict the dimension of the payoff table. The key observation is as follows: given a table $\mathrm{U}$ with maximum entry $\bar{m}$ and pure-strategy security level $v$, construct another payoff table $\mathrm{U}^{\prime}$ as follows: first, replace with $v$ the entries of $U$ that have values less than $v$. Then, add a new row of all $\underline{m} s$ to the resulting payoff table. Consider that $\mathrm{U}^{\prime}$ still has pure strategy security level of $v$ and maximum entry of $\bar{m}$. Furthermore, any strategy that guarantees payoff $w$ in game $\mathrm{U}$ also guarantees payoff $w$ in game $\mathrm{U}^{\prime}$.

Let us now turn to the third idea to prove a lower bound for $F_{\mathrm{U}}(w)$ in (37), namely replacing the set of distributions $\mathcal{P}_{\mathrm{U}}(w)$ with a potentially bigger set. As mentioned earlier, minimization of the entropy over the set

$$
\mathcal{P}_{\mathrm{U}}(w)=\left\{\mathbf{p} \in \Delta(\mathcal{A}): \mathbf{p}^{T} \mathrm{U} \geq w \mathbf{1}^{T}\right\}
$$


can be difficult. However, it could be possible to solve it (or find good lower bounds for it) for special choices of the matrix U. $4^{4}$ We show how a result for an special case of $\mathrm{U}$ can be utilized to find a bound (computable in polynomial time) for an arbitrary U. Assume that we have a way to minimize entropy over the set

$$
\mathcal{Q}(\mathbf{r}) \triangleq\left\{\mathbf{p} \in \Delta(\mathcal{A}): \mathbf{p}^{T} \mathrm{U}^{*} \geq \mathbf{r}^{T}\right\}
$$

for some given matrix $\mathrm{U}^{*}$, and any arbitrary column vector $\mathbf{r}$. We are interested in a value for $\mathbf{r}$ such that

$$
\mathcal{P}_{\mathrm{U}}(w) \subseteq \mathcal{Q}(\mathbf{r})
$$

We can relax the minimization of the entropy over the set $\mathcal{P}_{\mathrm{U}}(w)$ by computing its minimum over the bigger set of $\mathcal{Q}(\mathbf{r})$. Note that an appropriate $\mathbf{r}$ in $(43)$ can be found by solving a number of linear programs: the product $\mathbf{p}^{T} \mathrm{U}^{*}$ consists of a number of linear functions of coordinates of $\mathbf{p}$, and the minimum of each linear function over the set $\mathcal{P}_{\mathrm{U}}(w)$ is a linear program (see also Theorem 25).

Upper bound: It is clear that $F_{\mathrm{U}}(w) \leq H(\mathbf{p})$ for any arbitrary choice of $\mathbf{p} \in \mathcal{P}_{\mathrm{U}}(w)$. The following theorem gives a number of upper bounds each of which are obtained by identifying $\mathbf{p} \in \mathcal{P}_{\mathrm{U}}(w)$ in different ways.

Theorem 34. Consider a game with payoff matrix $\mathrm{U}$ and parameters $v, \underline{m}, \bar{m}$ and $w^{*}$ defined in Definition 21. Let $\mathrm{h}^{*}$ be the entropy of a Nash strategy of player A. Define

$$
\begin{aligned}
& Q_{\mathrm{U}}^{(1)}(w)=\min \left\{\mathrm{h}^{*}, \frac{w-v}{w^{*}-v} \mathrm{~h}^{*}+h\left(\frac{w-v}{w^{*}-v}\right)\right\}, \\
& Q_{\mathrm{U}}^{(2)}(w)=\min _{i \in \mathcal{A}} H\left(\mathbf{p}_{\text {max }, i}^{*}\right), \quad \mathbf{p}_{\text {max }, i}^{*} \in \underset{\mathbf{p} \in \mathcal{P}(w)}{\arg \max } p_{i}, \\
& Q_{\mathrm{U}}^{(3)}(w)=\min _{j \in \mathcal{B}} \quad \max _{\mathbf{p} \in \mathcal{P}_{\mathrm{U}}(w): \sum_{i \in \mathcal{A}} p_{i} u_{i, j}=w} H(\mathbf{p}),
\end{aligned}
$$

where $h(\alpha)=-\alpha \log (\alpha)-(1-\alpha) \log (1-\alpha)$ and $Q_{\mathrm{U}}^{(2)}(w)$ can be defined with any choice

\footnotetext{
${ }^{4}$ For instance, if each row of $U$ has only one non-zero element, the set of constraints will be on the individual coordinates of the vector $\mathbf{p}$ and minimizing entropy for such constraints is tractable.
} 
of $\mathbf{p}_{\text {max,i }}^{*}$ from the argmax set (if there are multiple possible choices). We have

$$
F_{\mathrm{U}}(w) \leq Q_{\mathrm{U}}^{(r)}(w), \quad r=1,2,3
$$

Proof of Theorem 34 can be found in Section 6.7 .

Remark 35. The second derivative of $Q_{\mathrm{U}}^{(1)}(w)$ is negative for $v \leq w \leq w^{*}$. Thus, $Q_{\mathrm{U}}^{(1)}(w)$ is a concave function of $w$ and one can readily inspect that:

$$
Q_{\mathrm{U} \oplus \mathrm{U}}^{(1)}(w)=Q_{\mathrm{U}}^{(1)}\left(\frac{w}{2}\right)
$$

As $\arg \max _{\mathbf{p} \in \mathcal{P}(w)} p_{i}$ may contain multiple elements, $Q_{\mathrm{U}}^{(2)}(w)$ is not a well defined function of $w$. The function $Q_{\mathrm{U}}^{(3)}(w)$ is not necessarily scalable for game $\mathrm{U} \oplus \mathrm{U}$.

Example 36. Consider two games with the following payoff tables:

$$
\mathrm{U}=\left[\begin{array}{cccccc}
3 & 1 & 0 & -2 & 0 & -2 \\
1 & 3 & -2 & 0 & -2 & 0 \\
0 & -2 & 3 & 1 & 0 & -2 \\
-2 & 0 & 1 & 3 & -2 & 0 \\
0 & -2 & 0 & -2 & 3 & 1 \\
-2 & 0 & -2 & 0 & 1 & 3
\end{array}\right], \quad \mathrm{U}^{\prime}=\left[\begin{array}{cccc}
0 & 1 & 1 & .5 \\
1 & 0 & .5 & 1 \\
1 & .5 & 0 & 1 \\
.5 & 1 & 1 & 0
\end{array}\right] .
$$

Figures 3 and 4 illustrate the behavior of the bounds for the games $\mathrm{U}$ and $\mathrm{U}^{\prime}$, respectively. In this examples, since $v=\underline{m}, G_{\mathrm{U}}^{(3)}(w)$ coincides with $G_{\mathrm{U}}^{(2)}(w)$, thus, it has not been depicted in the figures.

\section{Proofs}

\subsection{Proof of Proposition 12}

To prove Proposition 12 we make use of Lemmas 37 and 6. A brief discussion on randomness extraction and proof of Lemma 37 is provided in Appendix A. 


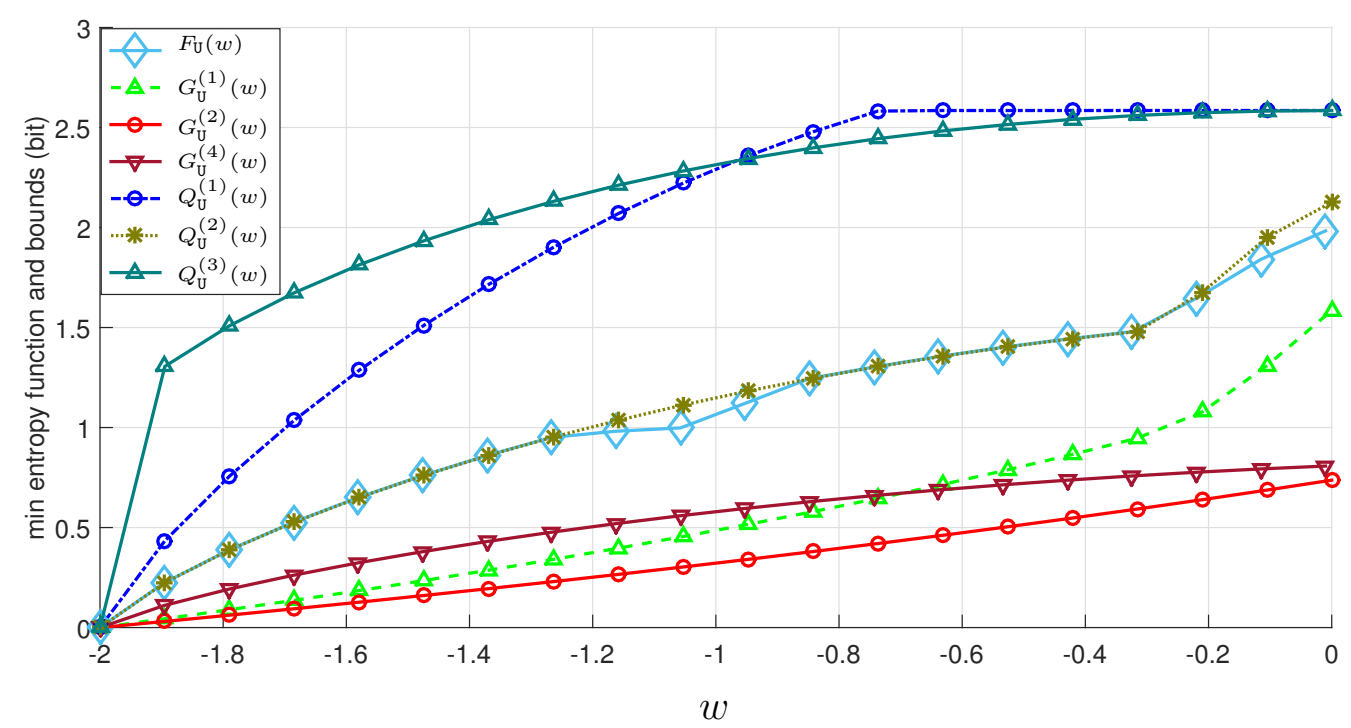

Figure 3: Illustration of the bounds on the min-entropy function for the game $\mathrm{U}$ defined in Example 36 . The horizontal line depicts $w$ and vertical line depicts the value of bounds.

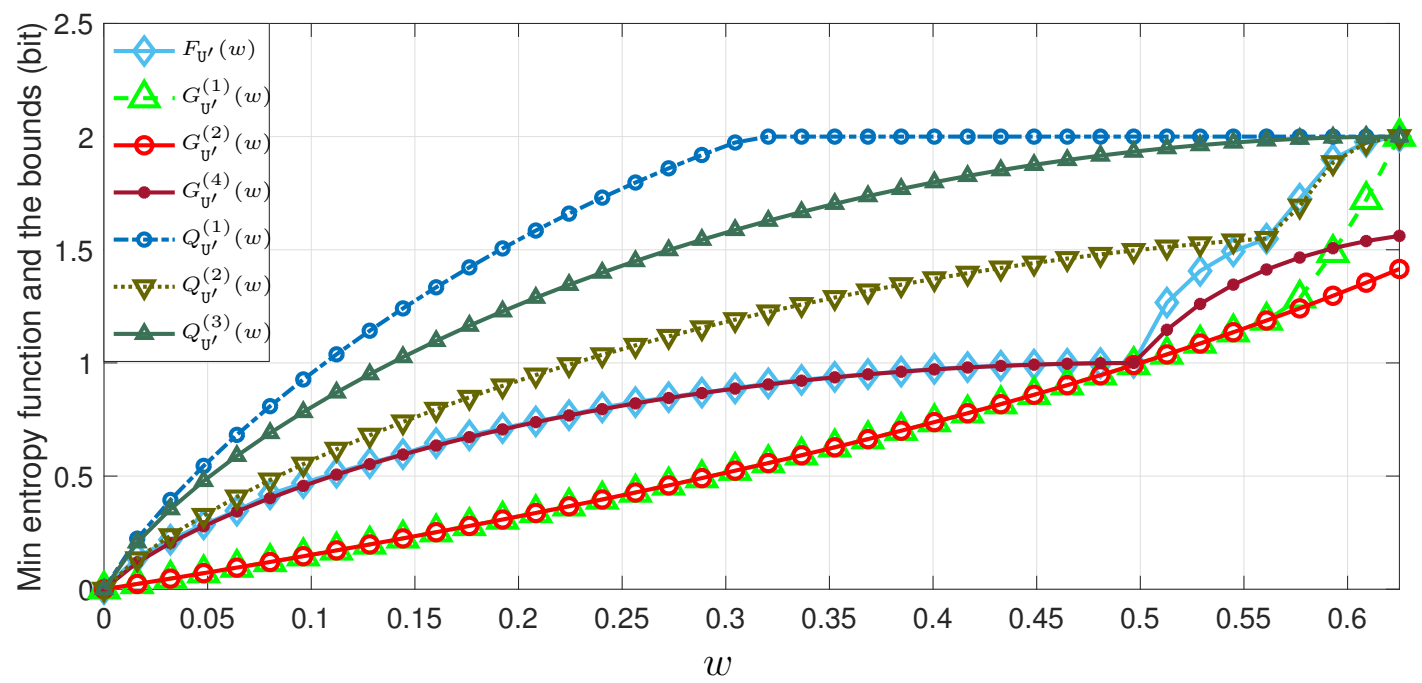

Figure 4: Illustration of the bounds on the min-entropy function for the game $\mathrm{U}^{\prime}$ defined in Example 36 . The horizontal line depicts $w$ and vertical line depicts the value of bounds. 
Lemma 37. Consider the correlated random sequences $X_{p}^{L}$ and $Y_{p}^{L}$ drawn i.i.d. from respective spaces $\mathcal{X}$ and $\mathcal{Y}$ by joint probability distribution $p_{X Y}$. Let $Q^{(L)}$ be a random variable independent of $Y_{p}^{L}$ and uniformly distributed on $\left\{1,2, \ldots, 2^{R L}\right\}$, where $R<H(X \mid Y)$ is a real number; then, there exist mappings $\mathrm{B}_{L}: \mathcal{X}^{L} \rightarrow\left\{1,2, \ldots, 2^{R L}\right\}$ such that

$$
\lim _{L \rightarrow \infty}\left\|p_{\mathrm{B}_{L}\left(X_{p}^{L}\right) Y_{p}^{L}}-p_{Q^{(L)} Y_{p}^{L}}\right\|_{T V}=0
$$

Choose a real number $R$ such that $\gamma H\left(p_{A}^{(1)}\right)+(1-\gamma) H\left(p_{A}^{(2)}\right)<R<H(X \mid Y)$ and $L R$ is a natural number (for a sufficiently large $L$ ).

We define random variables $Q^{(L)}$ and $\hat{A}^{L}$ that are mutually independent of each other and of $Y_{p}^{L}$ with the following marginal distributions: let $Q^{(L)}$ be a uniformly distributed random variable on $\left\{1,2, \ldots, 2^{R L}\right\}$ and $\hat{A}^{L}$ be distributed as follows:

$$
p_{\hat{A}^{L}}\left(a^{L}\right)=\prod_{t=1}^{\lceil\gamma L\rceil} p_{A}^{(1)}\left(a_{t}\right) \prod_{t=\lceil\gamma L)\rceil+1}^{L} p_{A}^{(2)}\left(a_{t}\right) .
$$

Note that $R<H(X \mid Y)$; thus, according to Lemma 37, there exist mappings $\mathrm{B}_{L}: \mathcal{X}^{L} \rightarrow$ $\left\{1,2, \ldots, 2^{R L}\right\}$ satisfying

$$
\lim _{L \rightarrow \infty}\left\|p_{\mathrm{B}_{L}\left(X_{p}^{L}\right) Y_{p}^{L}}-p_{Q^{(L)} Y_{p}^{L}}\right\|_{T V}=0
$$

On the other hand, we have

$$
\begin{aligned}
\mathrm{p}-\limsup _{L \rightarrow \infty} \frac{1}{L} \log \frac{1}{p_{\hat{A}^{L}}\left(\hat{A}^{L}\right)} & =\gamma H\left(p_{A}^{(1)}\right)+(1-\gamma) H\left(p_{A}^{(2)}\right) \\
& <R=\mathrm{p}-\liminf _{L \rightarrow \infty} \frac{1}{L} \log \frac{1}{p_{Q^{L}}\left(Q^{L}\right)}
\end{aligned}
$$

Therefore, according to Lemma 6, there exist mappings $\varphi_{L}:\left\{1,2, \ldots, 2^{R L}\right\} \rightarrow \mathcal{A}^{L}$ such that

$$
\lim _{L \rightarrow \infty}\left\|p_{\varphi_{L}\left(Q^{(L)}\right)}-p_{\hat{A}^{L}}\right\|_{T V}=0 .
$$

Considering the fact that $\varphi_{L}\left(Q^{(L)}\right)$ and $\hat{A}^{L}$ both are independent of $Y_{p}^{L}$, the above equation along with the third property of the total variation distance in Lemma 1 results in

$$
\lim _{L \rightarrow \infty}\left\|p_{\varphi_{L}\left(Q^{(L)}\right) Y_{p}^{L}}-p_{\hat{A}^{L} Y_{p}^{L}}\right\|_{T V}=0
$$


Furthermore, note that

$$
\begin{aligned}
\left\|p_{\varphi_{L}\left(\mathrm{~B}_{L}\left(X_{p}^{L}\right)\right) Y_{p}^{L}}-p_{\hat{A}^{L} Y_{p}^{L}}\right\|_{T V} & \leq\left\|p_{\varphi_{L}\left(\mathrm{~B}_{L}\left(X_{p}^{L}\right)\right) Y_{p}^{L}}-p_{\varphi_{L}\left(Q^{(L)}\right) Y_{p}^{L}}\right\|_{T V}+\left\|p_{\varphi_{L}\left(Q^{(L)}\right) Y_{p}^{L}}-p_{\hat{A}^{L} Y_{p}^{L}}\right\|_{T V} \\
& \leq\left\|p_{\mathrm{B}_{L}\left(X_{p}^{L}\right) Y_{p}^{L}}-p_{Q^{(L)} Y_{p}^{L}}\right\|_{T V}+\left\|p_{\varphi_{L}\left(Q^{(L)}\right) Y_{p}^{L}}-p_{\hat{A}^{L} Y_{p}^{L}}\right\|_{T V},
\end{aligned}
$$

where the first inequality follows from the triangle inequality for the total variation distance, and the second inequality follows from the fourth property of total variation in Lemma 1. Let $A_{c}^{L}=\psi_{L}\left(X_{p}^{L}\right)=\varphi_{L}\left(\mathrm{~B}_{L}\left(X_{p}^{L}\right)\right)$. Then, by combining Equations 44), 45) and (46), we have

$$
\lim _{L \rightarrow \infty}\left\|p_{A_{c}^{L} Y_{p}^{L}}-p_{\hat{A}^{L} Y_{p}^{L}}\right\|_{T V}=0 .
$$

Thus, $\psi_{L}(\cdot)=\varphi_{L}\left(\mathrm{~B}_{L}(\cdot)\right)$ is the desired mapping.

\subsection{Proof of Proposition 17}

Choose real numbers $R_{1}$ and $R_{2}$ such that

$$
R_{1}<H(X \mid Y, U=0), \quad R_{2}<H(X \mid Y, U=1), \quad p_{U}(0) R_{1}+p_{U}(1) R_{2}>H(Z \mid V),
$$

and for sufficiently large $L,\left\lceil p_{U}(0) L\right\rceil R_{1}$ and $\left\lceil p_{U}(1) L\right\rceil R_{2}$ are natural numbers. Since

$$
H(X \mid Y U)=p_{U}(0) H(X \mid Y, U=0)+p_{U}(1) H(X \mid Y, U=1)>H(Z \mid V),
$$

real numbers $R_{1}$ and $R_{2}$ with the above properties exist.

Let $\hat{L}=\left\lceil p_{U}(0) L\right\rceil$ and $\tilde{L}=L-\left\lceil p_{U}(0) L\right\rceil$. Observe that $L=\hat{L}+\tilde{L}$. We will consider three sets of random variables:

- (Set 1): Random variables $\left(X_{1}, \cdots, X_{L}, Y_{1}, \cdots, Y_{L}, W\right)$ defined in the statement of the proposition with the joint distribution $p_{X^{L} Y^{L} W}$ satisfying

$$
\left\|p_{X^{L} Y^{L} W}\left(x^{L}, y^{L}, w\right)-p_{W}(w) \prod_{t=1}^{\hat{L}} p_{X Y \mid U}\left(x_{t}, y_{t} \mid 0\right) \prod_{t=\hat{L}+1}^{L} p_{X Y \mid U}\left(x_{t}, y_{t} \mid 1\right)\right\|_{T V} \leq \delta_{1} .
$$


- (Set 2): Mutually independent random variables $W^{\prime},\left(\hat{X}^{\hat{L}}, \hat{Y}^{\hat{L}}\right)$ and $\left(\tilde{X}^{\tilde{L}}, \tilde{Y}^{\tilde{L}}\right)$. Here $p_{W^{\prime}}=p_{W}$. Furthermore, $\left(\hat{X}^{\hat{L}}, \hat{Y}^{\hat{L}}\right)$ and $\left(\tilde{X}^{\tilde{L}}, \tilde{Y}^{\tilde{L}}\right)$ are i.i.d. according to $p_{X Y \mid U}(\hat{x}, \hat{y} \mid 0)$ and $p_{X Y \mid U}(\tilde{x}, \tilde{y} \mid 1)$, respectively. In other words, for $X^{L}=\left(\hat{X}^{\hat{L}}, \tilde{X}^{\tilde{L}}\right)$ and $Y^{\prime L}=$ $\left(\hat{Y}^{\hat{L}}, \tilde{Y}^{\tilde{L}}\right)$ we have,

$$
p_{X^{\prime L} Y^{\prime L}}\left(x^{L}, y^{L}\right)=\prod_{t=1}^{\hat{L}} p_{X Y \mid U}\left(x_{t}, y_{t} \mid 0\right) \prod_{t=\hat{L}+1}^{L} p_{X Y \mid U}\left(x_{t}, y_{t} \mid 1\right) .
$$

Equation (48) along with the above equation and $p_{W^{\prime}}=p_{W}$ shows that the random variables in Set 2 are related to the random variables in Set 1 as follows:

$$
\left\|p_{X^{L} Y^{L} W}-p_{X^{\prime L} Y^{\prime L}} p_{W^{\prime}}\right\|_{T V} \leq \delta_{1}
$$

- (Set 3): Two independent random variables $\hat{Q}^{(\hat{L})}$ and $\tilde{Q}^{(\tilde{L})}$ with uniform distributions on $\left\{1,2, \ldots, 2^{\hat{L} R_{1}}\right\}$ and $\left\{1,2, \ldots, 2^{\tilde{L} R_{2}}\right\}$, respectively.

Since $R_{1}<H(X \mid Y, U=0)$ and $R_{2}<H(X \mid Y, U=1)$, according to Lemma 37, for arbitrary $\delta^{\prime}>0$, there exist mappings $\hat{\mathrm{B}}_{\hat{L}}: \mathcal{X}^{\hat{L}} \rightarrow\left\{1,2, \ldots, 2^{R_{1} \hat{L}}\right\}$ and $\tilde{\mathrm{B}}_{\tilde{L}}: \mathcal{X}^{\tilde{L}} \rightarrow$ $\left\{1,2, \ldots, 2^{R_{2} \tilde{L}}\right\}$ satisfying

$$
\left\|p_{\hat{\mathrm{B}}_{\hat{L}}\left(\hat{X}^{\hat{L}}\right) \hat{Y}^{\hat{L}}}-p_{\left.\hat{Q}^{(\hat{L})}\right)} p_{\hat{Y} \hat{L}}\right\|_{T V} \leq \delta^{\prime}, \quad\left\|p_{\tilde{\mathrm{B}}_{\tilde{L}}(\tilde{X} \tilde{L}) \tilde{Y} \tilde{L}}-p_{\tilde{Q}^{(\tilde{L})}} p_{\tilde{Y} \tilde{L}}\right\|_{T V} \leq \delta^{\prime},
$$

for sufficiently large $\hat{L}$ and $\tilde{L}$. Next, let $Q^{(L)}=\left(\hat{Q}^{(\hat{L})}, \tilde{Q}^{(\tilde{L})}\right)$, and $B_{L}: \mathcal{X}^{L} \rightarrow\left\{1,2, \ldots, 2^{R_{1} \hat{L}+R_{2} \tilde{L}}\right\}$ be the mapping $\mathrm{B}_{L}\left(\hat{X}^{\hat{L}}, \tilde{X}^{\tilde{L}}\right)=\left(\hat{\mathrm{B}}_{\hat{L}}\left(\hat{X}^{\hat{L}}\right), \tilde{\mathrm{B}}_{\tilde{L}}\left(\tilde{X}^{\tilde{L}}\right)\right)$. Using Equation (50), independence of $\left(\hat{X}^{\hat{L}}, \hat{Y}^{\hat{L}}\right)$ from $\left(\tilde{X}^{\tilde{L}}, \tilde{Y}^{\tilde{L}}\right)$, and independence of $\hat{Q}^{(\hat{L})}$ from $\tilde{Q}^{(\tilde{L})}$, we have

$$
\left\|p_{\mathrm{B}_{L}\left(\hat{X}^{\hat{L}}, \tilde{X}^{\tilde{L}}\right) \hat{Y}^{\hat{L}} \tilde{Y} \tilde{L}}-p_{Q^{(L)}} p_{\hat{Y}^{\hat{L}}} p_{\tilde{Y} \tilde{L}}\right\|_{T V} \leq 2 \delta^{\prime}
$$

where we used the third property of total variation in Lemma 1 for random variables $E_{1}=$ $\left(\hat{\mathrm{B}}_{\hat{L}}\left(\hat{X}^{\hat{L}}\right), \hat{Y}^{\hat{L}}\right), F_{1}=\left(\tilde{\mathrm{B}}_{\tilde{L}}\left(\tilde{X}^{\tilde{L}}\right), \tilde{Y}^{\tilde{L}}\right), E_{2}=\left(\hat{Q}^{(\hat{L})}, \hat{Y}^{\hat{L}}\right)$ and $F_{2}=\left(\tilde{Q}^{(\tilde{L})}, \tilde{Y}^{\tilde{L}}\right)$. Then, using the fifth property of total variation in Lemma 1 for random variables $E_{1}=\left(\mathrm{B}_{L}\left(\hat{X}^{\hat{L}}, \tilde{X}^{\tilde{L}}\right), \hat{Y}^{\hat{L}}, \tilde{Y}^{\tilde{L}}\right)$, $E_{2}=\left(Q^{(L)}, \hat{Y}^{\hat{L}}, \tilde{Y}^{\tilde{L}}\right)$ and $F=W^{\prime}$, we get

$$
\left\|p_{W^{\prime}} p_{\mathrm{B}_{L}\left(\hat{X}^{\hat{L}}, \tilde{X} \tilde{L}\right) \hat{Y}^{\hat{L}} \tilde{Y} \tilde{L}}-p_{W^{\prime}} p_{Q^{(L)}} p_{\hat{Y} \hat{L}} p_{\tilde{Y} \tilde{L}}\right\|_{T V} \leq 2 \delta^{\prime}
$$


Furthermore, by using the notation $X^{\prime L}=\left(\hat{X}^{\hat{L}}, \tilde{X}^{\tilde{L}}\right)$ and $Y^{\prime L}=\left(\hat{Y}^{\hat{L}}, \tilde{Y}^{\tilde{L}}\right)$, the above inequality is simplified as follows:

$$
\left\|p_{W^{\prime}} p_{\mathrm{B}_{L}\left(X^{\prime L}\right) Y^{\prime L}}-p_{W^{\prime}} p_{Q^{(L)}} p_{Y^{\prime L}}\right\|_{T V} \leq 2 \delta^{\prime}
$$

The above inequality shows that the mapping $\mathrm{B}_{L}$ simulates random variable $Q^{(L)}$ (in Set 3 of random variables) from $X^{L}$ (in Set 2 of random variables) within the total variation distance of $2 \delta^{\prime}$. Now, we consider the Set 1 of random variables. By applying the mapping $\mathrm{B}_{L}$ on sequence $X^{L}$ we have that

$$
\begin{gathered}
\left\|p_{W \mathrm{~B}_{L}\left(X^{L}\right) Y^{L}}-p_{W} p_{Q^{(L)}} p_{Y^{L}}\right\|_{T V} \leq\left\|p_{W \mathrm{~B}_{L}\left(X^{L}\right) Y^{L}}-p_{W^{\prime}} p_{\mathrm{B}_{L}\left(X^{\prime L}\right) Y^{\prime L}}\right\|_{T V}+ \\
\left\|p_{W^{\prime}} p_{\mathrm{B}_{L}\left(X^{\prime L}\right) Y^{\prime L}}-p_{W^{\prime}} p_{Q^{(L)}} p_{Y^{\prime L}}\right\|_{T V}+ \\
\left\|p_{W^{\prime}} p_{Q^{(L)}} p_{Y^{\prime L}}-p_{W} p_{Q^{(L)}} p_{Y^{L}}\right\|_{T V} \\
\leq 2 \delta_{1}+2 \delta^{\prime}
\end{gathered}
$$

where the first inequality follows from the triangle inequality for the total variation distance, and the second inequality results from Equation (51) and the following two facts:

- $\left\|p_{W \mathrm{~B}_{L}\left(X^{L}\right) Y^{L}}-p_{W^{\prime}} p_{\mathrm{B}_{L}\left(X^{\prime L}\right) Y^{\prime L}}\right\|_{T V} \leq \delta_{1}$. This is a consequence of Equation (49), combined with the fourth property of the total variation distance in Lemma 1.

- $\left\|p_{W^{\prime}} p_{Q^{(L)}} p_{Y^{\prime L}}-p_{W} p_{Q^{(L)}} p_{Y^{L}}\right\|_{T V} \leq \delta_{1}$. To see that this inequality is correct, first, note that Equation (49) along with the second property of total variation in Lemma 1 implies that $Y^{L}$ is in $\delta_{1}$ distance of $Y^{\prime L}$. Then, the fact that $p_{W^{\prime}}=p_{W}$ along with the fifth property of total variation in Lemma 1 gives the desired inequality.

Now, Let $\hat{Z}^{L}$ be an ideal sequence with distribution

$$
p_{\hat{Z}^{L}}\left(\hat{z}^{L}\right)=\prod_{t=1}^{\left\lceil p_{V}(0) L\right\rceil} p_{Z \mid V}\left(\hat{z}_{t} \mid 0\right) \prod_{t=\left\lceil p_{V}(0) L\right\rceil+1}^{L} p_{Z \mid V}\left(\hat{z}_{t} \mid 1\right) .
$$

Then, we have

$$
\begin{aligned}
\mathrm{p}-\limsup _{L \rightarrow \infty} \frac{1}{L} \log \frac{1}{p_{\hat{Z}^{L}}\left(\hat{Z}^{L}\right)} & =H(Z \mid V) \\
& <p_{U}(0) R_{1}+p_{U}(1) R_{2}=\mathrm{p}-\liminf _{L \rightarrow \infty} \frac{1}{L} \log \frac{1}{p_{Q^{L}}\left(Q^{L}\right)} .
\end{aligned}
$$


Therefore, according to Lemma 6 , there exist mappings $\varphi_{L}:\left\{1,2, \ldots, 2^{R_{1} \hat{L}+R_{2} \tilde{L}}\right\} \rightarrow \mathcal{Z}^{L}$ such that for sufficiently large $L$

$$
\left\|p_{\varphi_{L}\left(Q^{(L)}\right)}-p_{\hat{Z}^{L}}\right\|_{T V} \leq \delta^{\prime}
$$

Next, by replacing $p_{E_{1}}, p_{E_{2}}$ and $p_{F}$ with respective distributions $p_{\varphi_{L}\left(Q^{(L)}\right)}, p_{\hat{Z}^{L}}$ and $p_{W} p_{Y^{L}}$, the fifth property of total variation in Lemma 1 along with Equation (53) implies

$$
\left\|p_{\varphi_{L}\left(Q^{(L)}\right)} p_{Y^{L}} p_{W}-p_{\hat{Z}^{L}} p_{Y^{L}} p_{W}\right\|_{T V} \leq \delta^{\prime}
$$

Thus we have

$$
\begin{aligned}
\left\|p_{\varphi_{L}\left(\mathrm{~B}_{L}\left(X^{L}\right)\right) Y^{L} W}-p_{\hat{Z}^{L}} p_{Y^{L}} p_{W}\right\|_{T V} & \leq\left\|p_{\varphi_{L}\left(\mathrm{~B}_{L}\left(X^{L}\right)\right) Y^{L} W}-p_{\varphi_{L}\left(Q^{(L)}\right)} p_{Y^{L}} p_{W}\right\|_{T V}+ \\
& \quad\left\|p_{\varphi_{L}\left(Q^{(L)}\right)} p_{Y^{L}} p_{W}-p_{\hat{Z}^{L}} p_{Y^{L}} p_{W}\right\|_{T V} \\
& \leq\left\|p_{\mathrm{B}_{L}\left(X^{L}\right) Y^{L} W}-p_{Q^{(L)}} p_{Y^{L}} p_{W}\right\|_{T V}+\delta^{\prime} \\
& \leq 2 \delta_{1}+3 \delta^{\prime},
\end{aligned}
$$

where the first inequality follows from the triangle inequality for the total variation distance; the second inequality is a consequent of Equation (54) and the fourth property of total variation in Lemma 1 where $E_{1}$ and $E_{2}$ are replaced with $\mathrm{B}_{L}\left(X^{L}\right) Y^{L} W$ and $Q^{(L)} Y^{L} W$, respectively; finally, the third inequality results from Equation (52).

Let $Z^{L}=\varphi_{L}\left(\mathrm{~B}_{L}\left(X^{L}\right)\right)$ and take $\delta^{\prime}$ small enough that $3 \delta^{\prime} \leq \delta_{2}$. Then, from Equation 55) we have

$$
\left\|p_{Z^{L} Y^{L} W}-p_{\hat{Z}^{L}} p_{Y^{L}} p_{W}\right\|_{T V} \leq 2 \delta_{1}+\delta_{2}
$$

and the proof is complete.

\subsection{Proof of Theorems 22 and 23}

Take arbitrary $\mathbf{p} \in \mathcal{P}_{\mathrm{U}}^{c}\left(w_{2}\right)$ and $\mathbf{q} \in \mathcal{P}_{\mathrm{U}}\left(w_{1}\right)$. Since $\mathbf{p} \in \mathcal{P}_{\mathrm{U}}^{c}\left(w_{2}\right)$, when Alice plays according to $\mathbf{p}$, Bob has a (pure) strategy $j \in \mathcal{B}$ that reduces Alice's expected payoff to a number less than $w_{2}$. We can define a random variable $\tilde{W}$ that represents the payoff of Alice when Alice plays $\mathbf{p}$ and Bob plays $j$. Then, the alphabet set of $\tilde{W}$ is 
$\left\{u_{1, j}, u_{2, j}, \ldots, u_{n, j}\right\}$, and its probability distribution over this set is $\mathbf{p}$. We must have $\mathbb{E}[\tilde{W}]<w_{2}$

Let us assume that Alice adopts $\mathbf{q}$, but Bob keeps playing the same $j \in \mathcal{B}$. Let $W$ be the random variable describing the payoff of Alice when she plays according to $\mathbf{q}$. Because $\mathbf{q} \in \mathcal{P}_{\mathrm{U}}\left(w_{1}\right), \mathbb{E}[W] \geq w_{1}$. We can think of $W$ as taking $u_{i, j}$ with probability $q_{i}$.

Theorem 22 follows from the following chain of inequalities:

$$
\begin{aligned}
\left|w_{1}-w_{2}\right| & \leq|\mathbb{E}[W]-\mathbb{E}[\tilde{W}]| \\
& =\left|\sum_{i}\left(p_{i}-q_{i}\right) u_{i, j}\right| \\
& =\left|\sum_{i}\left(p_{i}-q_{i}\right)\left(u_{i, j}-\frac{\bar{m}+\underline{m}}{2}\right)\right| \\
& \leq\left(\sum_{i}\left|p_{i}-q_{i}\right|\right) \max _{i}\left|u_{i, j}-\frac{\bar{m}+\underline{m}}{2}\right| \\
& \leq\left(2 d_{1}(\mathbf{p}, \mathbf{q})\right)\left(\frac{|\bar{m}-\underline{m}|}{2}\right),
\end{aligned}
$$

where (56) follows from the fact that $(\bar{m}+\underline{m}) / 2$ is constant and $\sum_{i} p_{i}=\sum_{i} q_{i}=1$.

To prove Theorem 23, the key step is to relate the Rényi divergence to variance. Remember that $d_{2}(\mathbf{p}, \mathbf{q})=\log \left(1+\chi^{2}(\mathbf{p}, \mathbf{q})\right)$. The following lemma follows from the Chapman-Robbins bound:

Lemma 38. Take an arbitrary sequence of real numbers $\left(x_{1}, x_{2}, \ldots, x_{n}\right)$ and two probability distributions $\mathbf{p}=\left(p_{1}, p_{2}, \ldots, p_{n}\right)$ and $\mathbf{q}=\left(q_{1}, q_{2}, \ldots, q_{n}\right)$. Let $W$ be a random variable that takes value $x_{i}$ with probability $q_{i}$, and $\tilde{W}$ be a random variable that takes value $x_{i}$ with probability $p_{i}$. Then, we have

$$
\chi^{2}(\mathbf{p}, \mathbf{q}) \geq \frac{(\mathbb{E}[W]-\mathbb{E}[\tilde{W}])^{2}}{\operatorname{Var}[W]}
$$

Furthermore, the above inequality becomes an equality if we set $x_{i}=\left(p_{i}-q_{i}\right) / q_{i}$.

Observe that the left hand side of (58) depends only on the probability values $p_{i}$ and $q_{i}$, while the right hand side depends not only on the probabilities, but also the values 
that $W$ and $\tilde{W}$ take. Using this lemma and the fact that $\mathbb{E}[\tilde{W}]<w_{2}<w_{1} \leq \mathbb{E}[W]$, we can conclude

$$
\chi^{2}(\mathbf{p}, \mathbf{q}) \geq \frac{\left(\mathbb{E}[W]-w_{2}\right)^{2}}{\operatorname{Var}[W]}
$$

Observe that $\underline{m} \leq W \leq \bar{m}$ holds with probability one. The proof is finished by the following lemma.

Lemma 39. For any $w_{1}>w_{2}$, we have

$$
\frac{\left(\mathbb{E}[W]-w_{2}\right)^{2}}{\operatorname{Var}[W]} \geq \frac{\left(w_{1}-w_{2}\right)^{2}}{\left(w_{1}-\underline{m}\right)\left(\bar{m}-w_{1}\right)},
$$

provided that $\mathbb{E}[W] \geq w_{1}$, and $\underline{m} \leq W \leq \bar{m}$.

The proof of this lemma is given in Appendix C.

\subsection{Proof of Theorem 25}

In this section we provide additional details and build a geometric picture. This picture implies Theorem 25, but also gives a geometric interpretation of Nash strategies.

Let

$$
L\left(a_{1}, a_{2}, \ldots, a_{n}\right)=\operatorname{Val}\left(\mathrm{U}+\mathbf{a} 1^{T}\right), \quad \forall \mathbf{a} \in \mathbb{R}^{n} .
$$

Observe that the table $\tilde{U}$ can be intuitively understood as giving an additional incentive $a_{i}$ to Alice for playing her $i$-th action (it is actually a disincentive if $a_{i}<0$ ). Also, since

$$
L\left(a_{1}+c, a_{2}+c, \ldots, a_{n}+c\right)=L\left(a_{1}, a_{2}, \ldots, a_{n}\right)+c
$$

we only need to understand $L$ when the sum of the incentives $a_{i}$ is zero.

We need the following definition:

Definition 40. Let

$$
K\left(p_{1}, p_{2}, \ldots, p_{n}\right)=\min _{j} \sum_{i} p_{i} u_{i, j}
$$

be the payoff that Alice can guarantee with playing distribution $\left(p_{1}, \ldots, p_{n}\right)$ with table $\mathrm{U}$. We extend the definition of $K(\cdot)$ to arbitrary $\left(p_{1}, p_{2}, \ldots, p_{n}\right) \in \mathbb{R}^{n}$ by setting

$$
K\left(p_{1}, p_{2}, \ldots, p_{n}\right)=-\infty
$$


when the tuple $\left(p_{1}, p_{2}, \ldots, p_{n}\right)$ does not form a valid probability distribution, i.e., when any of the $p_{i}$ 's becomes negative, or $\sum_{i} p_{i} \neq 1$.

Note that

$$
\mathcal{P}_{\mathrm{U}}(w)=\{\mathbf{p} \mid K(\mathbf{p}) \geq w\} .
$$

A full geometric picture of $\mathcal{P}_{\mathrm{U}}(w)$ as well as Nash strategies are provided in the following theorem:

Theorem 41. We have

1. The function $L\left(a_{1}, a_{2}, \ldots, a_{n}\right)$ is the convex conjugate dual of $K\left(p_{1}, p_{2}, \ldots, p_{n}\right)$ in the following sense:

$$
L(\mathbf{a})=\max _{\mathbf{p} \in \mathbb{R}^{n}}\left[K(\mathbf{p})+\sum_{i=1}^{n} p_{i} a_{i}\right], \quad \forall \mathbf{a} \in \mathbb{R}^{n} .
$$

The function $L\left(a_{1}, a_{2}, \ldots, a_{n}\right)$ is jointly convex in $\left(a_{1}, a_{2}, \ldots, a_{n}\right)$, while $K\left(p_{1}, p_{2}, \ldots, p_{n}\right)$ is jointly concave in $\left(p_{1}, p_{2}, \ldots, p_{n}\right)$.

Furthermore, the supporting hyperplanes to the convex curve $\mathbf{a} \mapsto L(\mathbf{a})$ characterize Alice's Nash strategies as follows: for any arbitrary $\mathbf{a}, \mathbf{p}$ is a Nash strategy of Alice for table $\tilde{\mathrm{U}}=\mathrm{U}+\mathbf{a} \mathbf{1}^{T}$ if and only if $\mathbf{p}$ is a subgradient of the function $L$ at $\mathbf{a}$. In other words, take some arbitrary vector $\mathbf{a}$. Then,

$$
L\left(b_{1}, b_{2}, \ldots, b_{n}\right) \geq L\left(a_{1}, a_{2}, \ldots, a_{n}\right)+\sum_{i}\left(b_{i}-a_{i}\right) p_{i}, \quad \forall \mathbf{b} \in \mathbb{R}^{n},
$$

if and only if $\mathbf{p}$ is a Nash strategy of Alice for the payoff table $\tilde{\mathrm{U}}=\mathrm{U}+\mathbf{a} \mathbf{1}^{T}$.

2. Given a probability vector $\mathbf{p}$, we have

$$
L\left(a_{1}, a_{2}, \ldots, a_{n}\right) \geq w+\sum_{i}\left(a_{i}-b_{i}\right) p_{i}, \quad \forall \mathbf{a},
$$

if and only if $\mathbf{p}$ guarantees a payoff of at least $w$ for game $\mathbf{U}+\mathbf{b} \mathbf{1}^{T}$. In particular, setting $b_{i}=0$,

$$
L\left(a_{1}, a_{2}, \ldots, a_{n}\right) \geq w+\sum_{i} a_{i} p_{i}, \quad \forall \mathbf{a}
$$


if and only if $\mathbf{p}$ guarantees a payoff of at least $w$ for game $\mathrm{U}$, i.e., $\mathbf{p} \in \mathcal{P}_{\mathrm{U}}(w)$. Thus, having a payoff $w$, we look for hyperplanes of the form $w+\sum_{i} p_{i} a_{i}$ that pass through $w$ at $\left(a_{1}, a_{2}, \ldots, a_{n}\right)=(0,0, \ldots, 0)$, and lie below the curve of $L$.

Observe that the second part of Theorem 41 is equivalent with Theorem 25.

Proof of Theorem 41. We begin with the first part of the theorem. Using the max-min formulation for the value of a game $\tilde{\mathrm{U}}$, we have

$$
\begin{aligned}
L\left(a_{1}, a_{2}, \ldots, a_{n}\right) & =\max _{p_{i} \geq 0, \sum_{i} p_{i}=1} \min _{j} \sum_{i} p_{i}\left(u_{i, j}+a_{i}\right) \\
& =\max _{p_{i} \geq 0, \sum_{i} p_{i}=1}\left[\left(\min _{j} \sum_{i} p_{i} u_{i, j}\right)+\sum_{i} p_{i} a_{i}\right] \\
& =\max _{p_{i} \geq 0, \sum_{i} p_{i}=1}\left[K\left(p_{1}, p_{2}, \ldots, p_{n}\right)+\sum_{i} p_{i} a_{i}\right] \\
& =\max _{p_{i} \in \mathbb{R}}\left[K\left(p_{1}, p_{2}, \ldots, p_{n}\right)+\sum_{i} p_{i} a_{i}\right],
\end{aligned}
$$

where 62 follows from the fact that $K(\mathbf{p})$ is minus infinity when $\mathbf{p}$ is not a probability distribution. This shows the duality of $L(\cdot)$ and $K(\cdot)$. Next, note that $K(\cdot)$ is a minimum of linear functions; hence it is a concave function. Convexity of $L(\cdot)$ can be directly seen from 60 which implies that at least one supporting hyperplane to its curve exists at any given point (since at least one Nash strategy exists for any arbitrary game). Thus, it remains to prove 60 .

Without loss of generality, it suffices to prove 60 for $\mathbf{b}=\mathbf{0}\left(b_{i}=0\right)$, and get the result for arbitrary $\mathbf{b}$ by changing variable $\mathrm{U} \rightarrow \mathrm{U}+\mathbf{b} \mathbf{1}^{T}$. The inequality

$$
L\left(a_{1}, a_{2}, \ldots, a_{n}\right) \geq L(0,0, \ldots, 0)+\sum_{i} a_{i} p_{i}, \quad \forall \mathbf{a},
$$

can be also expressed as

$$
\min _{\mathbf{a}}\left(L\left(a_{1}, a_{2}, \ldots, a_{n}\right)-\sum_{i} a_{i} p_{i}\right) \geq L(0,0, \ldots, 0) .
$$


From the duality relation $(62)$ and utilizing the Fenchel's duality theorem, the left hand side is $K\left(p_{1}, p_{2}, \ldots, p_{n}\right)$. Thus, the expression is equivalent with 63 can be written as

$$
K\left(p_{1}, p_{2}, \ldots, p_{n}\right) \geq L(0,0, \ldots, 0)=\operatorname{Val}(\mathrm{U})
$$

which is equivalent with $\mathbf{p}$ being a Nash strategy.

The proof for the second part of the theorem is similar. As before $b_{i}$ can be set to zero. Then, we can express (61) as

$$
\min _{\mathbf{a}}\left(L\left(a_{1}, a_{2}, \ldots, a_{n}\right)-\sum_{i} a_{i} p_{i}\right) \geq w .
$$

From the duality relation $(62)$ and the Fenchel's duality theorem, this is equivalent with $K\left(p_{1}, p_{2}, \ldots, p_{n}\right) \geq w$, or $\mathbf{p} \in \mathcal{P}_{\mathrm{U}}(w)$.

\subsection{Proof of Theorem 28}

Let $p_{A}(a)$ be an arbitrary distribution that secures payoff $w / 2$ in game $\mathrm{U}$. Observe that $p_{A_{1} A_{2}}\left(a_{1}, a_{2}\right)=\mathbb{1}\left(a_{1}=a_{2}\right) p_{A}\left(a_{1}\right)$ secures payoff $w$ in game $\mathrm{U} \oplus \mathrm{U}$, where $\mathbb{1}(\cdot)$ is the indicator function. In this case, $H\left(p_{A_{1} A_{2}}\right)=H\left(p_{A}\right)$; thus, the function $F_{\mathrm{U} \oplus \mathrm{U}}(w)$ is bounded above as follows:

$$
F_{\mathrm{U} \oplus \mathrm{U}}(w) \leq F_{\mathrm{U}}\left(\frac{w}{2}\right) .
$$

On the other hand, let $p_{A_{1} A_{2}}^{*} \in \mathcal{P}_{\mathrm{U} \oplus \mathrm{U}}(w)$ be a distribution with minimum entropy that secures arbitrary payoff $w$ in game $\mathrm{U} \oplus \mathrm{U}$, i.e., $H\left(p_{A_{1} A_{2}}^{*}\right)=F_{\mathrm{U} \oplus \mathrm{U}}(w)$. Note that such a distribution $p_{A_{1} A_{2}}^{*}$ exists because $\mathcal{P}_{\mathrm{U} \oplus \mathrm{U}}(w)$ is compact. We have

$$
\begin{aligned}
w & \leq \min _{j_{1}, j_{2} \in \mathcal{B}} \mathbb{E}_{p_{A_{1} A_{2}}^{*}}\left[u_{A_{1}, j_{1}}+u_{A_{2}, j_{2}}\right] \\
& =\min _{j_{1}, j_{2} \in \mathcal{B}}\left(\mathbb{E}_{p_{A_{1} A_{2}}^{*}}\left[u_{A_{1}, j_{1}}\right]+\mathbb{E}_{p_{A_{1} A_{2}}^{*}}\left[u_{A_{2}, j_{2}}\right]\right) \\
& =\min _{j_{1} \in \mathcal{B}} \mathbb{E}_{p_{A_{1}}^{*}}\left[u_{A_{1}, j_{1}}\right]+\min _{j_{2} \in \mathcal{B}} \mathbb{E}_{p_{A_{2}}^{*}}\left[u_{A_{2}, j_{2}}\right],
\end{aligned}
$$

where $p_{A_{1}}^{*}$ and $p_{A_{2}}^{*}$ are the marginal distributions of $p_{A_{1} A_{2}}^{*}$. Equation (65) implies that there exists $k \in\{1,2\}$ such that

$$
\min _{j_{k} \in \mathcal{B}} \mathbb{E}_{p_{A_{k}}^{*}}\left[u_{A_{k}, j_{k}}\right] \geq \frac{w}{2}
$$


Thus, $p_{A_{k}}^{*}$ secures payoff $w / 2$ in game U. Hence,

$$
F_{\mathrm{U} \oplus \mathrm{U}}(w)=H\left(p_{A_{1} A_{2}}^{*}\right) \stackrel{(a)}{\geq} H\left(p_{A_{k}}^{*}\right) \geq F_{\mathrm{U}}\left(\frac{w}{2}\right),
$$

where $(a)$ follows from the properties of entropy.

Equations (64) and (66) conclude $F_{\mathrm{U} \oplus \mathrm{U}}(w)=F_{\mathrm{U}}(w / 2)$. The above line of proof can be extended for every natural number $k$ to prove $F_{\oplus^{k} \mathrm{U}}(w)=F_{\mathrm{U}}(w / k)$.

\subsection{Proof of Theorem 32}

If $v=\bar{m}$, then there exists a pure strategy $a \in \mathcal{A}$ that guarantees the maximum achievable payoff $\bar{m}$ in any permissible payoff table $\mathrm{U}^{\prime}$. Pure strategies have zero entropy and $G_{\mathrm{U}}^{(4)}(w)=0$. The explicit expression that we wish to prove for $G_{\mathrm{U}}^{(4)}(w)$ also vanishes as $v$ tends to $\bar{m}$, hence, the claim holds when $v=\bar{m}$. Therefore, for the rest of the proof we suppose that $v<\bar{m}$.

We claim that an optimal table for the minimization problem in the statement of the theorem is as follows:

$$
\mathrm{U}^{*}=\left[\begin{array}{cccccc}
\underline{m} & \bar{m} & \bar{m} & \bar{m} & \ldots & \bar{m} \\
\bar{m} & v & \bar{m} & \bar{m} & \ldots & \bar{m} \\
\bar{m} & \bar{m} & v & \bar{m} & \ldots & \bar{m} \\
\bar{m} & \bar{m} & \bar{m} & v & \ldots & \bar{m} \\
\vdots & \vdots & \vdots & \vdots & \ddots & \vdots \\
\bar{m} & \bar{m} & \bar{m} & \ldots & \ldots & v
\end{array}\right]_{m \times m}
$$

where the above square table is of size $m=\lfloor(\bar{m}-v) /(\bar{m}-w)\rfloor+2$. We first show that there is an optimal payoff table with the structure given in $(67)$ for some value of $m$ and then optimize over $m$, the size of the table.

Take some arbitrary feasible table $\mathrm{U}^{\prime}=\left[u_{i, j}^{\prime}\right]$ and distribution $\mathbf{p} \in \mathcal{P}_{\mathrm{U}^{\prime}}(w)$. Since $\min _{i \in \mathcal{A}} \min _{j \in \mathcal{B}} u_{i, j}^{\prime}=\underline{m}$, there exists a row $i$ and a column $j$ such that $u_{i, j}^{\prime}=\underline{m}$. By reordering the rows and columns of the table, we may assume that $u_{1,1}^{\prime}=\underline{m}$. Furthermore, since $\max _{i \in \mathcal{A}} \min _{j \in \mathcal{B}} u_{i, j}^{\prime}=v$, for all $i$, there exists a $j_{i}$ such that $u_{i, j_{i}}^{\prime} \leq v$. Thus, for all 
$i \in \mathcal{A}$ and $j \in \mathcal{B}$ we have $u_{i, j}^{\prime} \leq u_{i, j}^{\prime \prime}$, where $\mathrm{U}^{\prime \prime}=\left[u_{i, j}^{\prime \prime}\right]$ is defined as follows:

$$
u_{i, j}^{\prime \prime}= \begin{cases}\underline{m}, & i=1, j=1, \\ \bar{m}, & i=1, j \neq 1, \\ \bar{m}, & i \neq 1, j \neq j_{i}, \\ v, & i \neq 1, j=j_{i} .\end{cases}
$$

The table $\mathrm{U}^{\prime \prime}$ has higher payoffs for Alice than the table $\mathrm{U}^{\prime}$ in each cell. Hence, $\mathbf{p} \in \mathcal{P}_{\mathrm{U}^{\prime \prime}}(w)$. The table $\mathrm{U}^{\prime \prime}$ also belongs to the feasible set of the minimization problem because it has one row containing one entry with value $\underline{m}$ and all other rows contain an entry with value $v$.

Next, we can do two more operations: if there is a column with all $\bar{m}$ entries, we delete that column. Because we are restricting the action set of Bob, Alice can still secure payoff $w$ with the same distribution $\mathbf{p}$. If two rows of the table $\mathrm{U}^{\prime \prime}$ are the same, we can merge them together into one row. In other words, for instance the second and third rows are the same, i.e., $j_{2}=j_{3}$, we can merge the second and third rows together and assign the sum probability $p_{2}+p_{3}$ to this merged row. This will reduce the entropy of the probability vector $\mathbf{p}$ while leaving the payoff of Alice unchanged. With these two operations and row/column permutation, we either obtain the table given in 67) for some value of $m$, or the following $m \times(m-1)$ table

$$
\left[\begin{array}{cccccc}
\underline{m} & \bar{m} & \bar{m} & \bar{m} & \ldots & \bar{m} \\
v & \bar{m} & \bar{m} & \bar{m} & \ldots & \bar{m} \\
\bar{m} & v & \bar{m} & \bar{m} & \ldots & \bar{m} \\
\bar{m} & \bar{m} & v & \bar{m} & \ldots & \bar{m} \\
\vdots & \vdots & \vdots & \vdots & \ddots & \vdots \\
\bar{m} & \bar{m} & \bar{m} & \ldots & \ldots & v
\end{array}\right]_{m \times(m-1)}
$$

One can directly verify that any probability distribution $\mathbf{p}$ for the payoff of Alice from the table in 68 is less than or equal to the payoff of Alice from the table in (67). 
Having showed that a table with the structure given in $(67)$ is optimal, we now optimize over $m$, the size of the table, and compute $G_{\mathrm{U}}^{(4)}(w)$. The constraint $\mathbf{p}=\left(p_{1}, p_{2}, \ldots, p_{m}\right) \in$ $\mathcal{P}_{\mathrm{U}}(w)$ is equivalent to:

$$
\begin{aligned}
& \underline{m} p_{1}+\bar{m}\left(1-p_{1}\right) \geq w, \\
& v p_{i}+\bar{m}\left(1-p_{i}\right) \geq w, \quad \forall i>1,
\end{aligned}
$$

which simplifies to

$$
p_{1} \leq \frac{\bar{m}-w}{\bar{m}-\underline{m}}, \quad p_{i} \leq \frac{\bar{m}-w}{\bar{m}-v}, \quad \forall i>1 .
$$

We claim that without loss of generality, we can assume that $p_{1}=0$. Since $\frac{\bar{m}-w}{\bar{m}-v} \geq \frac{\bar{m}-w}{\bar{m}-\underline{m}}$, we have $p_{1} \leq \frac{\bar{m}-w}{\bar{m}-v}$. Now, if we have a distribution $\left(p_{1}, p_{2}, \ldots, p_{m}\right)$ on a table of the form (67) for some $m$, we can consider the distribution $\left(0, p_{1}, p_{2}, \ldots, p_{m}\right)$ on a table with size $m+1$; the entropy of $\left(0, p_{1}, p_{2}, \ldots, p_{m}\right)$ is the same as $\left(p_{1}, p_{2}, \ldots, p_{m}\right)$ and achieves the payoff of $w$. Therefore, the problem essentially reduces to the following: what is the minimum possible entropy for the set of all probability distributions $\left(p_{1}, p_{2}, \ldots, p_{m}\right)$ satisfying

$$
p_{1}=0, \quad p_{i} \leq \frac{\bar{m}-w}{\bar{m}-v}, \quad \forall i=2, \ldots, m .
$$

Note that we should choose $m$ large enough to ensure that the set of probability distributions satisfying (70) is non-empty. An optimal choice is to take $m=m^{*}$ for any $m^{*}$ satisfying $m^{*} \geq\lfloor(\bar{m}-v) /(\bar{m}-w)\rfloor+2$. The optimal pmf $\mathbf{p}^{*}$ is as follows:

$$
p_{i}^{*}= \begin{cases}(\bar{m}-w) /(\bar{m}-v), & i=2, \ldots,\lfloor(\bar{m}-v) /(\bar{m}-w)\rfloor+1, \\ 1-\lfloor(\bar{m}-v) /(\bar{m}-w)\rfloor(\bar{m}-w) /(\bar{m}-v), & i=\lfloor(\bar{m}-v) /(\bar{m}-w)\rfloor+2 \\ 0, & \text { otherwise. }\end{cases}
$$

The distribution $\mathbf{p}^{*}$ is the pmf that includes as much elements with value $(\bar{m}-w) /(\bar{m}-v)$ as possible. This distribution has minimum entropy because it majorizes all other pmfs in $\mathcal{P}_{\mathrm{U}}(w)$ Nielsen, 2002, Theorem 8.0.1). In other words, for any distribution $\mathbf{p}$ satisfying (70), and for all $i \leq m^{*}$, we have $\sum_{\ell=1}^{i} p_{\ell}^{* \downarrow} \geq \sum_{\ell=1}^{i} p_{\ell}^{\downarrow}$, where for a probability mass 
function $\mathbf{p}=\left(p_{1}, p_{2}, \ldots, p_{m}\right)$, we let $\mathbf{p}^{\downarrow}=\left(p_{1}^{\downarrow}, p_{2}^{\downarrow}, \ldots, p_{m}^{\downarrow}\right)$ be the vector whose elements are the elements of $\mathbf{p}$ reordered into non-decreasing order.

The entropy of $\mathbf{p}^{*}$ equals

$$
\begin{aligned}
& H\left(\mathbf{p}^{*}\right)=-\left\lfloor\frac{\bar{m}-v}{\bar{m}-w}\right\rfloor \frac{\bar{m}-w}{\bar{m}-v} \log \left(\frac{\bar{m}-w}{\bar{m}-v}\right)- \\
&\left(1-\left\lfloor\frac{\bar{m}-v}{\bar{m}-w}\right\rfloor \frac{\bar{m}-w}{\bar{m}-v}\right) \log \left(1-\left\lfloor\frac{\bar{m}-v}{\bar{m}-w}\right\rfloor \frac{\bar{m}-w}{\bar{m}-v}\right)
\end{aligned}
$$

as desired.

\subsection{Proof of Theorem 34}

Let $\mathbf{p}^{*}$ be a Nash strategy for player A where $H\left(\mathbf{p}^{*}\right)=\mathrm{h}^{*}$ and $i^{*} \in \arg \max _{i \in \mathcal{A}} \min _{j \in \mathcal{B}} u_{i, j}$ be the pure strategy that guarantees payoff $v$ for player A. For $v \leq w \leq w^{*}$ define $\alpha=\left(w^{*}-w\right) /\left(w^{*}-v\right)$ and $\mathbf{p}=\alpha \mathbf{e}_{i^{*}}+(1-\alpha) \mathbf{p}^{*}$ where $\mathbf{e}_{i^{*}}$ is a vector of all zero elements except for its $i^{*}$-th element which is 1 . The pmf $\mathbf{p}$ guarantees payoff $w$ since

$$
\mathbf{p}^{T} U=\alpha \mathbf{e}_{i^{*}}^{T} U+(1-\alpha) \mathbf{p}^{* T} U \geq \alpha v \mathbf{1}+(1-\alpha) w^{*} \mathbf{1}=w \mathbf{1}
$$

Using the properties of entropy one can inspect that:

$H(\mathbf{p}) \leq \alpha H\left(\mathbf{e}_{i^{*}}\right)+(1-\alpha) H\left(\mathbf{p}^{*}\right)+H(\alpha, 1-\alpha)=(1-\alpha) \mathbf{h}^{*}-\alpha \log _{2}(\alpha)-(1-\alpha) \log _{2}(1-\alpha)$.

Therefore, $F_{\mathrm{U}}(w) \leq H(\mathbf{p}) \leq(1-\alpha) \mathrm{h}^{*}-\alpha \log _{2}(\alpha)-(1-\alpha) \log _{2}(1-\alpha)$. In addition, as $\mathrm{h}^{*}$ secures payoff $w^{*} \geq w$, we have $F_{\mathrm{U}}(w) \leq \mathrm{h}^{*}$. These two facts imply $F_{\mathrm{U}}(w) \leq Q_{\mathrm{U}}^{(1)}(w)$ once we substitute the value of $\alpha$.

From the definition of $\mathbf{p}_{\max , i}^{*}$, it follows that $\mathbf{p}_{\max , i}^{*} \in \mathcal{P}_{\mathrm{U}}(w)$. Therefore, for every $i \in \mathcal{A}, F_{\mathrm{U}}(w) \leq H\left(\mathbf{p}_{\text {max }, i}^{*}\right)$ and $F_{\mathrm{U}}(w) \leq Q_{\mathrm{U}}^{(2)}(w)$ follows.

For an arbitrary $j \in \mathcal{B}$, if $\mathbf{p}^{\prime} \in\left\{\mathbf{p} \in \mathcal{P}_{\mathrm{U}}(w): \sum_{i \in \mathcal{A}} p_{i} u_{i, j}=w\right\}$, then, since $\mathbf{p}^{\prime}$ also belongs to $\mathcal{P}_{\mathrm{U}}(w)$, we have $F_{\mathrm{U}}(w) \leq H\left(\mathbf{p}^{\prime}\right)$. Hence, for every $j \in \mathcal{B}$ :

$$
F_{\mathrm{U}}(w) \leq \max _{\mathbf{p} \in \mathcal{P}_{\mathrm{U}}(w): \sum_{i \in \mathcal{A}} p_{i} u_{i, j}=w} H(\mathbf{p})
$$

The above inequality is correct for every $j \in \mathcal{B}$, thus, $F_{\mathrm{U}}(w) \leq Q_{\mathrm{U}}^{(3)}(w)$. 


\section{Appendix A. Review of the randomness extraction literature}

In this appendix, we review some results about randomness extraction which plays a key role in the construction of optimal strategies for the maximizer in the repeated games studied in this paper. The results of this section are adopted from Renner (2008) who considered the randomness extraction problem in the framework of quantum information theory.

Randomness extraction is the process of deriving some almost fair random bits from a given source $X$. More precisely, let $X \in \mathcal{X}$ and $Y \in \mathcal{Y}$ be two random variables with joint distribution $p_{X Y}$ and let $f: \mathcal{X} \rightarrow\left\{1,2,3, \ldots, 2^{\ell}\right\}$ be a deterministic mapping such that for $B=f(X)$ and $\epsilon>0$ we have

$$
\left\|p_{B Y}-p_{B}^{U} p_{Y}\right\|_{T V} \leq \epsilon
$$

where $p_{B Y}$ is the joint distribution of $B$ and $Y$ and $p_{B}^{U}$ is the uniform distribution on $\left\{1,2,3, \ldots, 2^{\ell}\right\}$. Then, we say $f$ extracts $\ell$ fair bits independent of $Y$ from $X$ with precision $\epsilon$.

To precede, we need the definition of conditional collision entropy for a given pair of random variables. The following subsection is devoted for this definition.

\section{Appendix A.1. Conditional collision entropy}

Let $X \in \mathcal{X}$ and $Y \in \mathcal{Y}$ be two random variables with joint probability distribution $p_{X Y}$, and let $q_{Y}$ be an arbitrary probability distribution on the sample space $\mathcal{Y}$. The conditional collision entropy of $p_{X Y}$ given $q_{Y}$ is defined as follows: $:^{5}$

$$
H_{2}\left(p_{X Y} \mid q_{Y}\right)=-\log \sum_{(x, y) \in \mathcal{X} \times \mathcal{Y}} \frac{p_{X Y}(x, y)^{2}}{q_{Y}(y)},
$$

and the conditional collision entropy of $p_{X Y}$ given $Y$ is defined as:

$$
H_{2}\left(p_{X Y} \mid Y\right)=\max _{q_{Y} \in \Delta(\mathcal{Y})} H_{2}\left(p_{X Y} \mid q_{Y}\right)
$$

\footnotetext{
${ }^{5}$ In this and upcoming definitions, we set $q_{Y}(y) / p_{X Y}(x, y)$ to be zero if $q_{Y}(y)=p_{X Y}(x, y)=0$, and infinity if $q_{Y}(y)>0$ while $p_{X Y}(x, y)=0$.
} 
The conditional collision entropy, as defined above, is not a continuous function of the input probability distribution and a slight change of the probability distribution might result in a large deviation in its conditional collision entropy. The smoothed version of conditional collision entropy resolves this drawback. Let $\epsilon$ be an arbitrary positive number and $p_{X Y}$ and $q_{Y}$ be some probability distributions on $\mathcal{X} \times \mathcal{Y}$ and $\mathcal{Y}$, respectively. The $\epsilon$-smooth conditional collision entropy of $p_{X Y}$ given $q_{Y}$ is

$$
H_{2}^{\epsilon}\left(p_{X Y} \mid q_{Y}\right)=\max _{\bar{p}_{X Y} \in \mathcal{B}_{\epsilon}\left(p_{X Y}\right)} H_{2}\left(\bar{p}_{X Y} \mid q_{Y}\right)
$$

where

$$
\mathcal{B}_{\epsilon}\left(p_{X Y}\right)=\left\{\bar{p}_{X Y} \in \Delta(\mathcal{X} \times \mathcal{Y}) ;\left\|\bar{p}_{X Y}-p_{X Y}\right\|_{T V} \leq \epsilon\right\}
$$

As before, the $\epsilon$-smooth conditional collision entropy of $p_{X Y}$ given $Y$ is defined as

$$
H_{2}^{\epsilon}\left(p_{X Y} \mid Y\right)=\max _{q_{Y} \in \Delta(\mathcal{Y})} H_{2}^{\epsilon}\left(p_{X Y} \mid q_{Y}\right)
$$

In the following remark, we show that for i.i.d. sources the smoothed conditional collision entropy is related to the Shannon entropy of the source.

Remark 42. Let $\left(X^{n}, Y^{n}\right) \in \mathcal{X}^{n} \times \mathcal{Y}^{n}$ be drawn i.i.d. from probability distribution $p_{X Y}=$ $p_{Y} p_{X \mid Y}$ and for arbitrary $\epsilon>0$, the set of typical sequences, $\tau_{\epsilon}^{(n)}$, be defined as

$$
\tau_{\epsilon}^{(n)}=\left\{\left(x^{n}, y^{n}\right) \in \mathcal{X}^{n} \times \mathcal{Y}^{n}:\left|-\frac{1}{n} \log p_{X^{n} \mid Y^{n}}\left(x^{n} \mid y^{n}\right)-H(X \mid Y)\right| \leq \epsilon\right\}
$$

where $p_{X^{n} \mid Y^{n}}\left(x^{n} \mid y^{n}\right)=\prod_{i=1}^{n} p_{X \mid Y}\left(x_{i} \mid y_{i}\right)$ is the conditional distribution of $X^{n}$ given $Y^{n}$. Considering that $\mathbb{E}\left[-\log p_{X \mid Y}(X \mid Y)\right]=H(X \mid Y)$ and

$$
-\frac{1}{n} \log p_{X^{n} \mid Y^{n}}\left(X^{n} \mid Y^{n}\right)=\frac{1}{n} \sum_{i=1}^{n}-\log p_{X \mid Y}\left(X_{i} \mid Y_{i}\right),
$$

the weak law of large numbers implies that for sufficiently large $n$,

$$
\operatorname{Pr}\left[\left(X^{n}, Y^{n}\right) \in \tau_{\epsilon}^{(n)}\right] \geq 1-\epsilon .
$$

Next, let $\bar{p}_{X^{n} Y^{n}}$ be a probability distribution on $\mathcal{X}^{n} \times \mathcal{Y}^{n}$ defined as

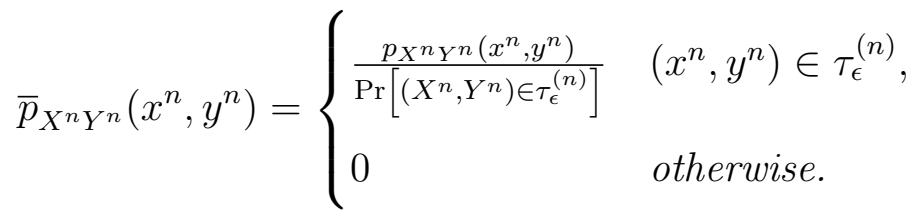


The definition above along with Equation (A.1) implies that $\left\|\bar{p}_{X^{n} Y^{n}}-p_{X^{n} Y^{n}}\right\|_{T V} \leq \epsilon$ holds for sufficiently large $n$. Therefore, for sufficiently large $n$, we have

$$
\begin{aligned}
H_{2}^{\epsilon}\left(p_{X^{n} Y^{n}} \mid Y^{n}\right) & \geq H_{2}\left(\bar{p}_{X^{n} Y^{n}} \mid \bar{p}_{Y^{n}}\right) \\
& =-\log \left(\sum_{\left(x^{n}, y^{n}\right) \in \mathcal{X}^{n} \times \mathcal{Y}^{n}} \bar{p}_{X^{n} Y^{n}}\left(x^{n}, y^{n}\right) \frac{\bar{p}_{X^{n} Y^{n}}\left(x^{n}, y^{n}\right)}{\bar{p}_{Y^{n}}\left(y^{n}\right)}\right) \\
& \geq-\log \left(\max _{\left(x^{n}, y^{n}\right) \in \mathcal{X}^{n} \times \mathcal{Y}^{n}} \bar{p}_{X^{n} \mid Y^{n}}\left(x^{n} \mid y^{n}\right)\right) \\
& =-\log \left(\max _{\left(x^{n}, y^{n}\right) \in \tau_{\epsilon}^{(n)}} p_{X^{n} \mid Y^{n}}\left(x^{n} \mid y^{n}\right)\right) \\
& \geq n(H(X \mid Y)-\epsilon) .
\end{aligned}
$$

\section{Appendix A.2. Randomness extraction}

In this subsection, we present the main tools regarding the extraction of randomness from a given source with known distribution.

Theorem 43 (Theorem 5.5.1, Renner (2008)). Let $X \in \mathcal{X}$ and $Y \in \mathcal{Y}$ be two random variables with joint distribution $p_{X Y}$. For arbitrary $\ell$ let $F: \mathcal{X} \rightarrow\left\{1,2,3, \ldots, 2^{\ell}\right\}$ be a random mapping constructed by assigning to $F(x)$ uniformly at random one element of $\left\{1,2,3, \ldots, 2^{\ell}\right\}$ and independently for distinct inputs $x, x^{\prime} \in \mathcal{X}$. Then, we have

$$
\sum_{f} p_{F}(f)\left\|p_{f(X) Y}-p_{B}^{U} p_{Y}\right\|_{T V} \leq \frac{1}{2} 2^{-\frac{1}{2}\left(H_{2}\left(p_{X Y} \mid Y\right)-\ell\right)}
$$

where the summation is over all deterministic mappings $f: \mathcal{X} \rightarrow\left\{1,2,3, \ldots, 2^{\ell}\right\}, p_{F}$ is the probability distribution of the random mapping $F, p_{f(X) Y}$ is the joint distribution of $Y$ and $f(X)$, and $p_{B}^{U}$ is the uniform distribution on the set $\left\{1,2,3, \ldots, 2^{\ell}\right\}$. Therefore, there exists a deterministic mapping $f: \mathcal{X} \rightarrow\left\{1,2,3, \ldots, 2^{\ell}\right\}$ such that

$$
\left\|p_{f(X) Y}-p_{B}^{U} p_{Y}\right\|_{T V} \leq \frac{1}{2} 2^{-\frac{1}{2}\left(H_{2}\left(p_{X Y} \mid Y\right)-\ell\right)} .
$$

Proof. To begin the proof we need a corollary of the Cauchy-Schwarz inequality stated in the following lemma. 
Lemma 44. Let $s_{1}, s_{2}, \ldots, s_{n}$ and $\gamma_{1}, \gamma_{2}, \ldots, \gamma_{n}$ be two sequences of real numbers such that for all $i=1,2, \ldots, n, \gamma_{i} \geq 0$ and $\sum_{i=1}^{n} \gamma_{i} \leq k$, where $k>0$. Then,

$$
\sum_{i=1}^{n}\left|s_{i}\right| \leq \sqrt{k\left(\sum_{i=1}^{n} \frac{s_{i}^{2}}{\gamma_{i}}\right)}
$$

where $0 / 0$ is taken as being zero and $1 / 0=\infty$.

Proof. If for some $i,\left|s_{i}\right|>0$ and $\gamma_{i}=0$ then the right hand side of the inequality in the statement of the lemma becomes infinity and hence it holds. Otherwise, let for all $i=1, \ldots, n$, if $\left|s_{i}\right|>0$, then $\gamma_{i}>0$; hence, by using Cauchy-Schwarz inequality we have

$$
\sum_{i=1}^{n}\left|s_{i}\right|=\sum_{i=1}^{n} \sqrt{\gamma_{i}} \frac{\left|s_{i}\right|}{\sqrt{\gamma_{i}}} \leq \sqrt{\left(\sum_{i=1}^{n} \gamma_{i}\right)\left(\sum_{i=1}^{n} \frac{s_{i}^{2}}{\gamma_{i}}\right)} \leq \sqrt{k\left(\sum_{i=1}^{n} \frac{s_{i}^{2}}{\gamma_{i}}\right)}
$$

where we take $0 / 0=0$ in accordance with the statement of the lemma.

Let $q_{Y}$ be an arbitrary probability distribution on $\mathcal{Y}$ and $f$ be an arbitrary realization of $F$, then, from Lemma 44 we have

$$
\begin{aligned}
\left\|p_{f(X) Y}-p_{B}^{U} p_{Y}\right\|_{T V} & =\frac{1}{2} \sum_{b=1}^{2^{\ell}} \sum_{y \in \mathcal{Y}}\left|p_{f(X) Y}(b, y)-p_{B}^{U}(b) p_{Y}(y)\right| \\
& \leq \frac{1}{2} \sqrt{2^{\ell}\left(\sum_{b=1}^{2^{\ell}} \sum_{y \in \mathcal{Y}} \frac{\left|p_{f(X) Y}(b, y)-p_{B}^{U}(b) p_{Y}(y)\right|^{2}}{q_{Y}(y)}\right)} \\
& =\frac{1}{2} \sqrt{2^{\ell}\left(\sum_{y \in \mathcal{Y}} \frac{p_{Y}(y)^{2}}{q_{Y}(y)} \sum_{b=1}^{2^{\ell}}\left|p_{f(X) \mid Y}(b \mid y)-p_{B}^{U}(b)\right|^{2}\right)}
\end{aligned}
$$


The term $\sum_{b=1}^{2^{\ell}}\left|p_{f(X) \mid Y}(b \mid y)-p_{B}^{U}(b)\right|^{2}$ is characterized as

$$
\begin{aligned}
\sum_{b=1}^{2^{\ell}} \mid p_{f(X) \mid Y}(b \mid y) & -\left.p_{B}^{U}(b)\right|^{2} \\
& =\sum_{b=1}^{2^{\ell}}\left|p_{f(X) \mid Y}(b \mid y)-2^{-\ell}\right|^{2} \\
& =\sum_{b=1}^{2^{\ell}}\left(p_{f(X) \mid Y}(b \mid y)^{2}-2 \times 2^{-\ell} p_{f(X) \mid Y}(b \mid y)+2^{-2 \ell}\right) \\
& =\left(\sum_{b=1}^{2^{\ell}} p_{f(X) \mid Y}(b \mid y)^{2}\right)-2^{-\ell} \\
& =\sum_{x, x^{\prime} \in \mathcal{X}} p_{X \mid Y}(x \mid y) p_{X \mid Y}\left(x^{\prime} \mid y\right)\left(\mathbb{1}\left(f(x)=f\left(x^{\prime}\right)\right)-2^{-\ell}\right) \\
& =\sum_{x \in \mathcal{X}} p_{X \mid Y}(x \mid y)^{2}\left(1-2^{-\ell}\right) \\
& \leq \sum_{x \in \mathcal{X}} p_{X \mid Y}(x \mid y)^{2}+\sum_{x \neq x^{\prime}} p_{X \mid Y}(x \mid y) p_{X \mid Y}\left(x^{\prime} \mid y\right)\left(\mathbb{1}\left(f(x)=f\left(x^{\prime}\right)\right)-2^{-\ell}\right)
\end{aligned}
$$

where $\mathbb{1}(\cdot)$ is the indicator function and Equation $A$ A.3 is implied by the following fact: $\sum_{b=1}^{2^{\ell}}\left(-2 \times 2^{-\ell} p_{f(X) \mid Y}(b \mid y)+2^{-2 \ell}\right)=-2 \times 2^{-\ell}\left(\sum_{b=1}^{2^{\ell}} p_{f(X) \mid Y}(b \mid y)\right)+2^{-2 \ell} \times 2^{\ell}=-2 \times 2^{-\ell}+2^{-\ell}=-2^{-\ell}$, where we used $\sum_{b=1}^{2^{\ell}} p_{f(X) \mid Y}(b \mid y)=1$. To justify Equation A.4 , note that $\sum_{b=1}^{2^{\ell}} p_{f(X) \mid Y}(b \mid y)^{2}$ is the probability of the event $f\left(X^{\prime}\right)=f\left(X^{\prime \prime}\right)$, where $X^{\prime}$ and $X^{\prime \prime}$ are i.i.d. random variables with distribution $p_{X \mid Y=y}$, i.e., $p_{X^{\prime} X^{\prime \prime}}\left(x, x^{\prime}\right)=p_{X \mid Y}(x \mid y) p_{X \mid Y}\left(x^{\prime} \mid y\right)$. On the other hand, the probability of the event $f\left(X^{\prime}\right)=f\left(X^{\prime \prime}\right)$ is also characterized as

$$
\sum_{x, x^{\prime} \in \mathcal{X}} p_{X \mid Y}(x \mid y) p_{X \mid Y}\left(x^{\prime} \mid y\right) \mathbb{1}\left(f(x)=f\left(x^{\prime}\right)\right) .
$$

Thus,

$$
\sum_{b=1}^{2^{\ell}} p_{f(X) \mid Y}(b \mid y)^{2}=\sum_{x, x^{\prime} \in \mathcal{X}} p_{X \mid Y}(x \mid y) p_{X \mid Y}\left(x^{\prime} \mid y\right) \mathbb{1}\left(f(x)=f\left(x^{\prime}\right)\right) .
$$


The above equation along with the fact that $\sum_{x, x^{\prime} \in \mathcal{X}} p_{X \mid Y}(x \mid y) p_{X \mid Y}\left(x^{\prime} \mid y\right)=1$ implies Equation (A.4.

Next, by taking the average of $\sum_{b=1}^{2^{\ell}}\left|p_{f(X) \mid Y}(b \mid y)-p_{B}^{U}(b)\right|^{2}$ with respect to $F$ we have

$$
\begin{aligned}
\sum_{f} p_{F}(f) & \sum_{b=1}^{2^{\ell}}\left|p_{f(X) \mid Y}(b \mid y)-p_{B}^{U}(b)\right|^{2} \\
& \leq \sum_{x \in \mathcal{X}} p_{X \mid Y}(x \mid y)^{2}+\sum_{x \neq x^{\prime}} p_{X \mid Y}(x \mid y) p_{X \mid Y}\left(x^{\prime} \mid y\right)\left(\sum_{f} p_{F}(f) \mathbb{1}\left(f(x)=f\left(x^{\prime}\right)\right)-2^{-\ell}\right) \\
& =\sum_{x \in \mathcal{X}} p_{X \mid Y}(x \mid y)^{2}+\sum_{x \neq x^{\prime}} p_{X \mid Y}(x \mid y) p_{X \mid Y}\left(x^{\prime} \mid y\right)\left(\operatorname{Pr}\left[F(x)=F\left(x^{\prime}\right)\right]-2^{-\ell}\right) \\
& =\sum_{x \in \mathcal{X}} p_{X \mid Y}(x \mid y)^{2},
\end{aligned}
$$

where the inequality follows from (A.5), and the last equality holds because for distinct $x, x^{\prime} \in \mathcal{X}, F(x)$ coincides with $F\left(x^{\prime}\right)$ with probability $2^{-\ell}$. Therefore,

$$
\begin{aligned}
\sum_{f} p_{F}(f)\left\|p_{f(X) Y}-p_{B}^{U} p_{Y}\right\|_{T V} & \leq \frac{1}{2} \sum_{f} p_{F}(f) \sqrt{2^{\ell}\left(\sum_{y \in \mathcal{Y}} \frac{p_{Y}(y)^{2}}{q_{Y}(y)} \sum_{b=1}^{2^{\ell}}\left|p_{f(X) \mid Y}(b \mid y)-p_{B}^{U}(b)\right|^{2}\right)} \\
& \leq \frac{1}{2} \sqrt{2^{\ell}\left(\sum_{y \in \mathcal{Y}} \frac{p_{Y}(y)^{2}}{q_{Y}(y)} \sum_{f} p_{F}(f) \sum_{b=1}^{2^{\ell}}\left|p_{f(X) \mid Y}(b \mid y)-p_{B}^{U}(b)\right|^{2}\right)} \\
& \leq \frac{1}{2} \sqrt{2^{\ell\left(\sum_{y \in \mathcal{Y}} \frac{p_{Y}(y)^{2}}{q_{Y}(y)} \sum_{x \in \mathcal{X}} p_{X \mid Y}(x \mid y)^{2}\right)}} \\
& =\frac{1}{2} \sqrt{\left.2^{\ell} \sum_{(x, y) \in \mathcal{X} \times \mathcal{Y}} \frac{p_{X Y}(x, y)^{2}}{q_{Y}(y)}\right)} \\
& =\frac{1}{2} 2^{-\frac{1}{2}\left(H_{2}\left(p_{X Y} \mid q_{Y}\right)-\ell\right)},
\end{aligned}
$$

where the first inequality follows from A.2, the second inequality follows from utilizing the Jensen's inequality for concave function $\sqrt{\cdot}$, the third inequality follows from (A.6), and the second equality follows from the definition of $H_{2}\left(p_{X Y} \mid q_{Y}\right)$. Since $q_{Y}$ is arbitrary, the claim of the theorem is implied by the above inequality. 
Now, we can utilize Theorem 43 to obtain another bound in terms of the smoothed conditional collision entropy. Let $\epsilon>0$ be an arbitrary real number, and $X \in \mathcal{X}$ and $Y \in$ $\mathcal{Y}$ be two random variables with joint probability distribution $p_{X Y}$. Let $\bar{p}_{X Y} \in \mathcal{B}_{\epsilon}\left(p_{X Y}\right)$ be the probability distribution such that

$$
H_{2}^{\epsilon}\left(p_{X Y} \mid Y\right)=H_{2}\left(\bar{p}_{X Y} \mid Y\right)
$$

and $f: \mathcal{X} \rightarrow\left\{1,2,3, \ldots, 2^{\ell}\right\}$ be the mapping of Theorem 43 satisfying

$$
\left\|\bar{p}_{f(X) Y}-p_{B}^{U} \bar{p}_{Y}\right\|_{T V} \leq \frac{1}{2} 2^{-\frac{1}{2}\left(H_{2}\left(\bar{p}_{X Y} \mid Y\right)-\ell\right)}=\frac{1}{2} 2^{-\frac{1}{2}\left(H_{2}^{\epsilon}\left(p_{X Y} \mid Y\right)-\ell\right)},
$$

where $\bar{p}_{f(X) Y}$ is the joint distribution of $(f(X), Y)$ when $(X, Y)$ is distributed according to $\bar{p}_{X Y}$, and $\bar{p}_{Y}$ is the marginal distribution of $\bar{p}_{X Y}$ with respect to $Y$. Then, we have

$$
\begin{aligned}
\left\|p_{f(X) Y}-p_{B}^{U} p_{Y}\right\|_{T V} & \leq\left\|p_{f(X) Y}-\bar{p}_{f(X) Y}\right\|_{T V}+\left\|\bar{p}_{f(X) Y}-p_{B}^{U} \bar{p}_{Y}\right\|_{T V}+\left\|p_{B}^{U} \bar{p}_{Y}-p_{B}^{U} p_{Y}\right\|_{T V} \\
& \leq 2 \epsilon+\frac{1}{2} 2^{-\frac{1}{2}\left(H_{2}^{\epsilon}\left(p_{X Y} \mid Y\right)-\ell\right)},
\end{aligned}
$$

where the first inequality results from the triangular inequality for the total variation distance, and the second inequality follows from A.7 and the following two facts:

- $\left\|p_{f(X) Y}-\bar{p}_{f(X) Y}\right\|_{T V} \leq\left\|p_{X Y}-\bar{p}_{X Y}\right\|_{T V} \leq \epsilon$ : This is a consequence of the forth property of the total variation distance in Lemma 1 .

- $\left\|p_{B}^{U} \bar{p}_{Y}-p_{B}^{U} p_{Y}\right\|_{T V}=\left\|\bar{p}_{Y}-p_{Y}\right\|_{T V} \leq\left\|p_{X Y}-\bar{p}_{X Y}\right\|_{T V} \leq \epsilon$ : The equality and the first inequality follow from the fifth and the second property (respectively) of the total variation distance in Lemma 1 .

Therefore, the following corollary of Theorem 43 is concluded.

Corollary 45. For random variables $X \in \mathcal{X}$ and $Y \in \mathcal{Y}$ with joint distribution $p_{X Y}$ and $\epsilon>0$, there exists a deterministic mapping $f: \mathcal{X} \rightarrow\left\{1,2, \ldots, 2^{\ell}\right\}$ such that

$$
\left\|p_{f(X) Y}-p_{B}^{U} p_{Y}\right\|_{T V} \leq 2 \epsilon+\frac{1}{2} 2^{-\frac{1}{2}\left(H_{2}^{\epsilon}\left(p_{X Y} \mid Y\right)-\ell\right)}
$$

where $p_{B}^{U}$ is the uniform distribution on $\left\{1,2, \ldots, 2^{\ell}\right\}$. 
Next, we utilize Corollary 45 in combination with Remark 42 to conclude that for the random sequence $\left(X^{n}, Y^{n}\right)$ drawn i.i.d. from $p_{X Y}$ there exists a mapping that extracts almost $n H(X \mid Y)$ bits from $X^{n}$ with desired precision given that $n$ is sufficiently large. This is the claim of Lemma 37 in Section 6.1. We finish this section by restating Lemma 37 and providing its formal proof.

Lemma 37. Consider the correlated random sequences $X^{n}$ and $Y^{n}$ drawn i.i.d. from respective spaces $\mathcal{X}$ and $\mathcal{Y}$ by joint probability distribution $p_{X Y}$. Let $Q^{(n)}$ be a random variable independent of $Y^{n}$ and uniformly distributed on $\left\{1,2, \ldots, 2^{R n}\right\}$, where $R<H(X \mid Y)$ is a real number; then, there exist mappings $\mathrm{B}_{n}: \mathcal{X}^{n} \rightarrow\left\{1,2, \ldots, 2^{R n}\right\}$ such that

$$
\lim _{n \rightarrow \infty}\left\|p_{\mathrm{B}_{n}\left(X^{n}\right) Y^{n}}-p_{Q^{(n)}} p_{Y^{n}}\right\|_{T V}=0 .
$$

Proof. For arbitrary $\delta>0$ choose some positive number $\epsilon$ such that $\epsilon<\delta / 2$ and $\epsilon<$ $H(X \mid Y)-R$. Let $\mathrm{B}_{n}$ be the mapping of Corollary 45 satisfying

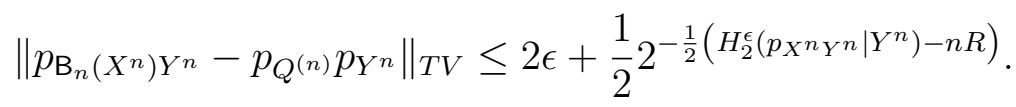

Then, by using Remark 42 , for sufficiently large $n$ we have

$$
\left\|p_{\mathrm{B}_{n}\left(X^{n}\right) Y^{n}}-p_{Q^{(n)}} p_{Y^{n}}\right\|_{T V} \leq 2 \epsilon+\frac{1}{2} 2^{-\frac{n}{2}(H(X \mid Y)-R-\epsilon)} .
$$

Since $\epsilon<\delta / 2$ and $H(X \mid Y)-R-\epsilon>0$, there exists a natural number $n_{0}$ such that for all $n>n_{0}, 2 \epsilon+\frac{1}{2} 2^{-\frac{n}{2}(H(X \mid Y)-R-\epsilon)} \leq \delta$; hence, for sufficiently large $n$,

$$
\left\|p_{\mathrm{B}_{n}\left(X^{n}\right) Y^{n}}-p_{Q^{(n)}} p_{Y^{n}}\right\|_{T V} \leq \delta
$$

which finishes the proof.

\section{Appendix B. Proof of Proposition 20}

First, we show that the cardinality of random variable $R$ can be reduced to two. Without loss of generality, we may assume that $p_{R}(r)>0$ for all $r \in \mathcal{R}$. We will alter the joint distribution $p_{R Q \mathbf{A} S}$ and construct a new joint distribution $p_{R Q \mathbf{A} S}^{\prime}$ on the same alphabet sets such that 
- $p_{R Q \mathbf{A} S}^{\prime}$ still satisfies the constraints (12)-(14) and (30);

- $p_{R}^{\prime}(r)>0$ for only two values of $r$, i.e., the support of $R$ under distribution $p_{R Q \mathbf{A} S}^{\prime}$ is of cardinality at most two.

Let

$$
p_{Q \mathbf{A} S \mid R}^{\prime}(q, \mathbf{a}, s \mid r)=p_{Q \mathbf{A} S \mid R}(q, \mathbf{a}, s \mid r), \quad \forall(q, \mathbf{a}, s, r) .
$$

In other words, the distributions $p$ and $p^{\prime}$ differ only in the marginal distribution of $R$. Observe that with this choice of $p_{Q \mathbf{A} S \mid R}^{\prime}=p_{Q \mathbf{A} S \mid R}$, Equations 12 and (13) are satisfied for $p_{R Q \mathbf{A} S}^{\prime}$ regardless of how we choose the marginal distribution of $R$; these properties are inherited from $p_{R Q \mathbf{A} S}$.

To specify the joint distribution $p_{R Q \mathbf{A} S}^{\prime}$, we need to specify $p_{R}^{\prime}(r)$ for different values of $r \in \mathcal{R}$. We can think of the marginal distribution $p_{R}^{\prime}$ as a vector of size $|\mathcal{R}|$ of non-negative numbers adding up to one, i.e., a vector $\left[p_{R}^{\prime}(r), r \in \mathcal{R}\right]$ in the probability simplex

$$
\begin{aligned}
& p_{R}^{\prime}(r) \geq 0, \quad, \forall r \in \mathcal{R}, \\
& \sum_{r \in \mathcal{R}} p_{R}^{\prime}(r)=1 .
\end{aligned}
$$

Let $H(. \mid$.$) and H^{\prime}(. \mid$.$) denote the entropy function under distributions p_{R Q \mathbf{A} S}$ and $p_{R Q \mathbf{A} S}^{\prime}$, respectively. Similarly, let $\pi(\mathbf{A} \mid R)$ and $\pi^{\prime}(\mathbf{A} \mid R)$ represent the security level of $\mathbf{A}$ given $R$ under distributions $p_{R Q \mathbf{A} S}$ and $p_{R Q \mathbf{A} S}^{\prime}$, respectively. The expression

$$
H^{\prime}(Q \mathbf{A} \mid S R)-H^{\prime}(Q \mid R)=\sum_{r \in \mathcal{R}} p_{R}^{\prime}(r)\left[H^{\prime}(Q \mathbf{A} \mid S, R=r)-H^{\prime}(Q \mid R=r)\right]
$$

is linear in $p_{R}^{\prime}(r)$. We impose the following linear constraint on $p_{R}^{\prime}(r)$ :

$$
\sum_{r \in \mathcal{R}} p_{R}^{\prime}(r)\left[H^{\prime}(Q \mathbf{A} \mid S, R=r)-H^{\prime}(Q \mid R=r)\right]=H(Q \mathbf{A} \mid S R)-H(Q \mid R)
$$

This linear constraint implies that $H^{\prime}(Q \mathbf{A} \mid S R)-H^{\prime}(Q \mid R)=H(Q \mathbf{A} \mid S R)-H(Q \mid R)$. This would ensure (14) for $p_{R Q \mathbf{A} S}^{\prime}$. Now, consider the polytope $\mathrm{P}$ formed by real vectors $\left[p_{R}^{\prime}(r), r \in \mathcal{R}\right]$ of size $|\mathcal{R}|$ satisfying $(\mathrm{B} .1)-(\mathrm{B} .3)$. This polytope is non-empty as it includes the vector corresponding to the marginal distribution $\left[p_{R}(r)\right]$. The expression $\pi^{\prime}(\mathbf{A} \mid R)$ is 
also a linear expression in $p_{R}^{\prime}(r)$ for $r \in \mathcal{R}$. The maximum of the linear function $\pi^{\prime}(\mathbf{A} \mid R)$ over polytope $\mathrm{P}$ occurs at a vertex of $\mathrm{P}$. We choose the distribution corresponding to this vertex. Thus, $\pi^{\prime}(\mathbf{A} \mid R) \geq \pi(\mathbf{A} \mid R)$ and (30) is satisfied under $p_{R Q \mathbf{A} S}^{\prime}$. Next, it suffices to show that each vertex of $\mathrm{P}$ corresponds to a joint distribution $p_{R}^{\prime}(r)$ with at most two nonnegative entries. The polytope $P$ is defined via $|\mathcal{R}|+2$ hyperplanes given in (B.1)-(B.3). Since $\mathrm{P}$ lies in a space of dimension $|\mathcal{R}|$, each of its vertices must lie at the intersection of at least $|\mathcal{R}|$ hyperplanes defining $P$. In other words, each vertex must lie on at least $|\mathcal{R}|$ of the $|\mathcal{R}|+2$ hyperplanes given in $(\mathrm{B} .1)-(\mathrm{B} .3)$. This implies that each vertex must lie on at least $|\mathcal{R}|-2$ hyperplanes of the type given in (B.1). In other words, each vertex must have at least $|\mathcal{R}|-2$ zero entries, and hence at most two non-zero entries.

Next, we alter the joint distribution $p_{R Q \mathbf{A} S}^{\prime}$ and construct a new joint distribution $p_{R Q \mathbf{A} S}^{\prime \prime}$ on the same alphabet sets such that

- $p_{R Q \mathbf{A} S}^{\prime \prime}$ still satisfies the constraints 12$)-(14)$ and (30);

- $p_{Q}^{\prime \prime}(q)>0$ for at most $2|\mathcal{A}|$ values of $q$, i.e., the size of the support of $Q$ under distribution $p_{R Q \mathbf{A} S}^{\prime \prime}$ is at most $2|\mathcal{A}|$.

Let

$$
p_{R}^{\prime \prime}(r)=p_{R}^{\prime}(r), \quad p_{\mathbf{A} S \mid R Q}^{\prime \prime}(\mathbf{a}, s \mid r, q)=p_{\mathbf{A} S \mid R Q}^{\prime}(\mathbf{a}, s \mid r, q), \quad \forall(\mathbf{a}, s, r, q) .
$$

In other words, the marginal distribution on $R$ and the conditional distribution on $\mathbf{A} S$ given $R Q$ are preserved. We will now choose $p_{Q \mid R}^{\prime \prime}$ to fulfill the definition of the joint distribution $p^{\prime \prime}$. Equation (B.4) implies that Equations (12) and (13) are satisfied under $p^{\prime \prime}$. For arbitrary $r \in \mathcal{R}$ satisfying $p_{R}^{\prime \prime}(r)>0$, as before, we interpret $p_{Q \mid R=r}^{\prime \prime}$ as a real vector of size $|\mathcal{Q}|$ in the probability simplex

$$
\begin{aligned}
& p_{Q \mid R=r}^{\prime \prime}(q) \geq 0, \quad \forall q \in \mathcal{Q}, \\
& \sum_{q \in \mathcal{Q}} p_{Q \mid R=r}^{\prime \prime}(q)=1 .
\end{aligned}
$$


Note that $p_{\mathbf{A} \mid R=r}^{\prime \prime}(\mathbf{a})=\sum_{q \in \mathcal{Q}} p_{Q \mid R=r}^{\prime \prime}(q) p_{\mathbf{A} \mid Q, R=r}^{\prime \prime}(\mathbf{a} \mid q)$ is a linear function of $p_{Q \mid R=r}^{\prime \prime}$, so we impose the following linear constraints on $p_{Q \mid R=r}^{\prime \prime}$ :

$$
p_{\mathbf{A} \mid R=r}^{\prime \prime}(\mathbf{a})=p_{A \mid R=r}^{\prime}(\mathbf{a}), \quad \forall \mathbf{a} \in \mathcal{A}-\left\{\mathbf{a}_{0}\right\}
$$

where $\mathbf{a}_{0}$ is an arbitrary element of $\mathcal{A}$ and $\mathcal{A}-\left\{\mathbf{a}_{0}\right\}$ is the set $\mathcal{A}$ from which $\mathbf{a}_{0}$ is excluded. Consider that as $p_{\mathbf{A} \mid R=r}^{\prime \prime}$ and $p_{\mathbf{A} \mid R=r}^{\prime}$ both sum up to one, Equation (B.7) guarantees that $p_{\mathbf{A} \mid R=r}^{\prime \prime}\left(\mathbf{a}_{0}\right)=p_{A \mid R=r}^{\prime}\left(\mathbf{a}_{0}\right)$, thus $p_{\mathbf{A} \mid R=r}^{\prime \prime}=p_{\mathbf{A} \mid R=r}^{\prime}$. The fact that $p_{\mathbf{A} \mid R=r}^{\prime \prime}=p_{\mathbf{A} \mid R=r}^{\prime}$, along with Equation (B.4), implies

$$
\begin{aligned}
& \pi^{\prime \prime}(\mathbf{A} \mid R)=\pi^{\prime}(\mathbf{A} \mid R), \\
& H^{\prime \prime}(S \mid R)=H^{\prime}(S \mid R),
\end{aligned}
$$

where $H^{\prime \prime}\left(. \mid\right.$.) denotes the entropy function and $\pi^{\prime \prime}(\mathbf{A} \mid R)$ denotes the security level of $\mathbf{A}$ given $R$, both under the distribution $p_{R Q \mathbf{A} S}^{\prime \prime}$. Equation (B.8) implies that Equation (30) is satisfied under $p_{R Q \mathbf{A} S}^{\prime \prime}$. Let $\mathrm{P}^{\prime}$ denote the polytope of all conditional distributions $p_{Q \mid R=r}^{\prime \prime}$ satisfying B.5 - B.7). Note that $\mathrm{P}^{\prime}$ is non-empty since it includes $p_{Q \mid R=r}^{\prime}$. We choose $p_{Q \mid R=r}^{\prime \prime}$ to be a vertex of $\mathrm{P}^{\prime}$ that maximizes $H^{\prime \prime}(\mathbf{A} S \mid Q, R=r)$ (linear in $\left.p_{Q \mid R=r}^{\prime \prime}\right)$. Therefore, we have $H^{\prime \prime}(\mathbf{A} S \mid Q, R=r) \geq H^{\prime}(\mathbf{A} S \mid Q, R=r)$ and considering that $p_{R}^{\prime \prime}=p_{R}^{\prime}$, we conclude that

$$
H^{\prime \prime}(\mathbf{A} S \mid Q R) \geq H^{\prime}(\mathbf{A} S \mid Q R)
$$

Then, using Equations (B.9) and (B.10), we have

$$
\begin{aligned}
H^{\prime \prime}(Q \mathbf{A} \mid R S)-H^{\prime \prime}(Q \mid R) & =H^{\prime \prime}(\mathbf{A} S \mid R Q)-H^{\prime \prime}(S \mid R) \\
& \geq H^{\prime}(\mathbf{A} S \mid R Q)-H^{\prime}(S \mid R) \\
& =H^{\prime}(Q \mathbf{A} \mid R S)-H^{\prime}(Q \mid R) \\
& \geq 0,
\end{aligned}
$$

where the equations follow from the properties of the entropy function, the first inequality follows from Equations (B.9) and (B.10), and the last inequality follows from the fact that $p^{\prime}$ satisfies (14). Thus, Equation (14) is satisfied under $p_{R Q \mathbf{A} S}^{\prime \prime}$. Next, we complete the 
proof by showing that the support of $Q$ under the distribution $p_{R Q \mathbf{A} S}^{\prime \prime}$ has cardinality of at most $2|\mathcal{A}|$. To do this, it suffices to show that for arbitrary $r \in \mathcal{R}$ for which $p_{R}^{\prime \prime}(r)>0$, the conditional distribution $p_{Q \mid R=r}^{\prime \prime}$ has cardinality at most $|\mathcal{A}|$. Then, from the fact that $p_{R}^{\prime \prime}(r)>0$ for only two values of $r \in \mathcal{R}$, we can conclude that under $p_{R Q \mathbf{A} S}^{\prime \prime}$, the support of $Q$ has cardinality at most $2|\mathcal{A}|$. Note that the polytope $\mathrm{P}^{\prime}$ belongs to a space of dimension $|Q|$, thus, every vertex of $\mathrm{P}^{\prime}$ lies in the intersection of at least $|Q|$ hyperplanes defining $\mathrm{P}^{\prime}$ (Equations (B.5)-(B.7)). On the other hand, $\mathrm{P}^{\prime}$ is defined by $|\mathcal{Q}|+|\mathcal{A}|$ hyperplanes of which $|\mathcal{Q}|$ hyperplanes are of the type given in $(\mathrm{B} .5)$; thus, every vertex of $\mathrm{P}^{\prime}$ lies in at least $|\mathcal{Q}|-|\mathcal{A}|$ hyperplanes of the type given in $(\mathrm{B} .5)$. Therefore, $p_{Q \mid R=r}^{\prime \prime}$ has at least $|\mathcal{Q}|-|\mathcal{A}|$ zero-valued entries and hence at most $|\mathcal{A}|$ non-zero entries.

\section{Appendix C. Proof of Lemma 39}

Let us minimize the expression $\left(\mathbb{E}[W]-w_{2}\right)^{2} / \operatorname{Var}[W]$ over all random variables $W$ that satisfy $\underline{m} \leq W \leq \bar{m}$ and $\mathbb{E}[W] \geq w_{1}$ :

$$
\begin{aligned}
\min _{\substack{m \leq W \leq \bar{m} \\
\mathbb{E}[W] \geq w_{1}}} \frac{\left(\mathbb{E}[W]-w_{2}\right)^{2}}{\operatorname{Var}[W]} & =\min _{w_{1} \leq \mu \leq \bar{m}}\left(\min _{\substack{m \leq W \leq \bar{m} \\
\mathbb{E}[W]=\mu}} \frac{\left(\mathbb{E}[W]-w_{2}\right)^{2}}{\operatorname{Var}[W]}\right)=\min _{w_{1} \leq \mu \leq \bar{m}}\left(\frac{\left(\mu-w_{2}\right)^{2}}{\left.\max _{\substack{m \leq W \leq \bar{m} \\
\mathbb{E}[W]=\mu}}^{\operatorname{Var}[W]}\right)}\right. \\
& =\min _{w_{1} \leq \mu \leq \bar{m}}\left(\frac{\left(\mu-w_{2}\right)^{2}}{-\mu^{2}+\max _{\substack{m \leq W \leq \bar{m} \\
\mathbb{E}[W]=\mu}} \mathbb{E}\left[W^{2}\right]}\right)
\end{aligned}
$$

We claim that

$$
\max _{\substack{m \leq W \leq \bar{m} \\ \mathbb{E}[W]=\mu}} \mathbb{E}\left[W^{2}\right]=(\bar{m}-\mu)(\mu-\underline{m})+\mu^{2} .
$$

Observe that if $W^{*}$ is the following binary random variable

$$
\mathbb{P}\left[W^{*}=\underline{m}\right]=\frac{\bar{m}-\mu}{\bar{m}-\underline{m}}, \quad \mathbb{P}\left[W^{*}=\bar{m}\right]=\frac{\mu-\underline{m}}{\bar{m}-\underline{m}},
$$

we have $\mathbb{E}\left[W^{*}\right]=\mu$ and $\mathbb{E}\left[\left(W^{*}\right)^{2}\right]=(\bar{m}-\mu)(\mu-\underline{m})+\mu^{2}$. As a result,

$$
\max _{\substack{m \leq W \leq \bar{m} \\ \mathbb{E}[W]=\mu}} \mathbb{E}\left[W^{2}\right] \geq(\bar{m}-\mu)(\mu-\underline{m})+\mu^{2} .
$$


On the other hand, the function $f(x)=x^{2}$ is convex and lies below the line that connects the two points $\left(\underline{m}, \underline{m}^{2}\right)$ and $\left(\bar{m}, \bar{m}^{2}\right)$ for any $x \in[\underline{m}, \bar{m}]$, i.e.,

$$
x^{2} \leq \underline{m}^{2}+(x-\underline{m})(\bar{m}+\underline{m}), \quad \forall x \in[\underline{m}, \bar{m}] .
$$

Thus,

$$
\mathbb{E}\left[W^{2}\right] \leq \underline{m}^{2}+(\mathbb{E}[W]-\underline{m})(\bar{m}+\underline{m})=(\bar{m}-\mu)(\mu-\underline{m})+\mu^{2} .
$$

Thus, Equation C.2 holds. As a result, Equation (C.1) becomes

$$
\min _{\substack{m \leq W \leq \bar{m} \\ \mathbb{E}[W] \geq w_{1}}} \frac{\left(\mathbb{E}[W]-w_{2}\right)^{2}}{\operatorname{Var}[W]}=\min _{w_{1} \leq \mu \leq \bar{m}} g(\mu),
$$

where

$$
g(\mu)=\frac{\left(\mu-w_{2}\right)^{2}}{(\bar{m}-\mu)(\mu-\underline{m})} .
$$

Note that the function $g(\cdot)$ is increasing for any $\mu$ satisfying $\underline{m} \leq w_{2} \leq \mu \leq \bar{m}$ as

$$
\frac{d g(\mu)}{d \mu}=\frac{\left(\mu-w_{2}\right)\left[(\bar{m}-\mu)\left(\mu+w_{2}-2 \underline{m}\right)+(\mu-\underline{m})\left(\mu-w_{2}\right)\right]}{(\bar{m}-\mu)^{2}(\mu-\underline{m})^{2}} \geq 0 .
$$

Since $w_{2} \leq w_{1}$, this would then imply that the minimum on the right hand side of (C.5) is obtained at $\mu=w_{1}$. This will complete the proof.

\section{Acknowledgements}

The authors would like to thank Yury Polyanskiy for pointing out the connection with the ChapmanRobbins bound. The authors also want to thank the anonymous reviewers for their helpful suggestions. This work was supported by the Sharif University of Technology under Grant QB950607.

\section{References}

Adler, I., 2013. The equivalence of linear programs and zero-sum games. International Journal of Game Theory 42 (1), 165-177. 
Budinich, M., Fortnow, L., 2011. Repeated matching pennies with limited randomness. In: Proceedings of the 12th ACM conference on Electronic commerce. ACM, pp. 111-118.

Cicalese, F., Gargano, L., Vaccaro, U., 2017. How to find a joint probability distribution of minimum entropy (almost), given the marginals. arXiv preprint arXiv:1701.05243.

Cover, T. M., Thomas, J. A., 2012. Elements of information theory. John Wiley \& Sons.

Dantzig, G. B., 1951. A proof of the equivalence of the programming problem and the game problem. Activity analysis of production and allocation (13), 330-338.

El Gamal, A., Kim, Y.-H., 2011. Network information theory. Cambridge university press.

Fang, S.-C., Rajasekera, J. R., Tsao, H.-S. J., 2012. Entropy optimization and mathematical programming. Vol. 8. Springer Science \& Business Media.

Gossner, O., Laraki, R., Tomala, T., 2009. Informationally optimal correlation. Mathematical Programming 116 (1-2), 147-172.

Gossner, O., Tomala, T., 2007. Secret correlation in repeated games with imperfect monitoring. Mathematics of Operations Research 32 (2), 413-424.

Gossner, O., Vieille, N., 2002. How to play with a biased coin? Games and Economic Behavior 41 (2), 206-226.

Han, T. S., 2003. Information-spectrum methods in information theory. Vol. 50. Springer -Verlag Berlin Heidelberg.

Hubáček, P., Naor, M., Ullman, J., 2016. When can limited randomness be used in repeated games? Theory of Computing Systems 59 (4), 722-746.

Kapur, J. N., Baciu, G., Kesavan, H. K., 1995. The minmax information measure. International journal of systems science 26 (1), 1-12. 
Kocaoglu, M., Dimakis, A. G., Vishwanath, S., Hassibi, B., 2017. Entropic causal inference. In: AAAI. pp. 1156-1162.

Kovačević, M., Stanojević, I., Šenk, V., 2012. On the hardness of entropy minimization and related problems. In: Information Theory Workshop (ITW), 2012 IEEE. IEEE, pp. $512-516$.

Kovačević, M., Stanojević, I., Šenk, V., 2015. On the entropy of couplings. Information and Computation 242, 369-382.

Narahari, Y., 2014. Game theory and mechanism design. Vol. 4. World Scientific.

Nash, J. F., 1950. Equilibrium points in n-person games. Proceedings of the national academy of sciences 36 (1), 48-49.

Neyman, A., Okada, D., 2000. Repeated games with bounded entropy. Games and Economic Behavior 30 (2), 228-247.

Nielsen, M. A., 2002. An introduction to majorization and its application to quantum mechanics. Lecture Notes, Department of physics, University of Queensland, Australia.

Renner, R., 2008. Security of quantum key distribution. International Journal of Quantum Information 6 (01), 1-127.

Shor, P. W., 2004. Equivalence of additivity questions in quantum information theory. Communications in Mathematical Physics 246 (3), 453-472.

Slepian, D., Wolf, J., 1973. Noiseless coding of correlated information sources. IEEE Transactions on information Theory 19 (4), 471-480.

Srikanth, M., Kesavan, H., Roe, P., 2003. Computation of the minmax measure. In: Entropy Measures, Maximum Entropy Principle and Emerging Applications. Springer, pp. 239-251. 
Watanabe, S., 1981. Pattern recognition as a quest for minimum entropy. Pattern Recognition 13 (5), 381-387. 